\title{
TIMETABLE-BASED ROUTING IN FIXED SCHEDULE DYNAMIC NETWORKS
}

\author{
Cristian Oswaldo Rodriguez Santiago
}

\author{
A THESIS \\ IN \\ The Department \\ $\mathrm{OF}$ \\ Computer Science And Software Engineering
}

Presented in Partial Fulfillment of the Requirements

For the Degree of Master of Computer Science CONCORDIA UNIVERSITY Montréal, Québec, Canada

MARCH 2021

(C) Cristian Oswaldo Rodriguez Santiago, 2021 


\section{CONCORDIA UNIVERSITY \\ School of Graduate Studies}

This is to certify that the thesis prepared

By: $\quad$ Cristian Oswaldo Rodriguez Santiago

Entitled: Timetable-based Routing in Fixed Schedule Dynamic Networks

and submitted in partial fulfillment of the requirements for the degree of

\section{Master of Computer Science}

complies with the regulations of this University and meets the accepted standards with respect to originality and quality.

Signed by the final examining commitee:

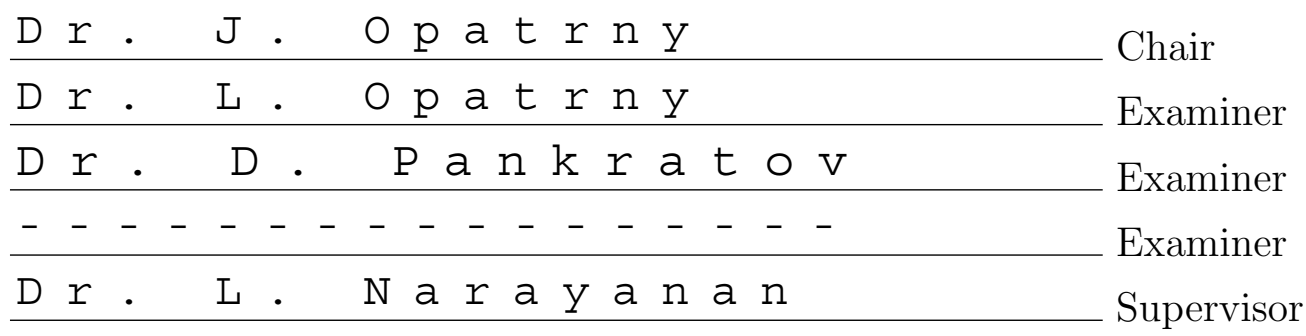

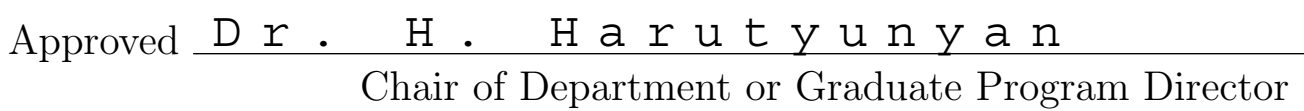

March 25th 2021

Mourad Debbabi, Ph.D., Interim Dean

Faculty of Engineering and Computer Science 


\title{
Abstract \\ Timetable-based Routing in Fixed Schedule Dynamic Networks \\ Cristian Oswaldo Rodriguez Santiago
}

\begin{abstract}
A fixed schedule dynamic network has a set of nodes (eg. vehicles or satellites) that move using a known schedule and trajectory, so that the connections between nodes in the network appear and disappear in a predictable manner. We study the problem of finding a foremost journey in such a network: given a query time, source and destination node, we find a temporal path that arrives at the earliest time at the destination. We give a new approach to the problem that uses a timetable sorted in order of the start time of connections, and describe three algorithms using this approach. We prove their correctness and give tight bounds on their worst-case time complexities. We also show extensive experimental results that show that our new algorithms outperforms the previous best algorithm given in [21].
\end{abstract}




\section{Acknowledgments}

I thank my God for providing me the strength and courage to complete this work.

I would like to express a sincere gratitude and appreciation to my supervisor Dr. Lata Narayanan; for her patience, dedication and commitment.

Thanks to all my family, especially my mother Adelina and my sister Camila, for their love and support. I also would like to manifest a deep appreciation to Dr. Luis Felipe Urquiza, a generous and good friend of mine.

To all my brothers and sisters in Christ members of First Filipino Baptist Church of Montréal and Bible Study Fellowship of Montréal, thank you all for your prayers. 


\section{Contents}

List of Figures vii

List of Tables $\quad$ ix

1 Introduction 1

1.1 Motivation . . . . . . . . . . . . . . . . . 1

1.2 Problem definition . . . . . . . . . . . . . . . . . . 3

1.3 Our results . . . . . . . . . . . . . . . . . . 4

1.4 Organization .......................... 5

2 Related work $\quad 6$

2.1 Dynamic graph models . . . . . . . . . . . . . . . 6

2.1.1 Snapshots . . . . . . . . . . . . . . 7

2.1.2 Whole graph .................. 8

$2.1 .3 \log$ file. . . . . . . . . . . . . . . 8

2.1.4 Distributed graph over servers . . . . . . . . . . . . . . 9

2.2 Journey computation . . . . . . . . . . . . . . . . . . . . . 9 9

2.2.1 Dijkstra's algorithms extensions . . . . . . . . . . . . . 11

2.3 Connection scan algorithm . . . . . . . . . . . . . . . . . . . . . . 12

3 Timetable-Based journey computation 16

3.1 Preliminaries . . . . . . . . . . . . . . . . . . 16

3.1 .1 Evolving graph . . . . . . . . . . . . . . . 16

3.1 .2 Connection . . . . . . . . . . . . . . 20

3.1.3 Timetable ... . . . . . . . . . . . . . . 20

3.1.4 Duplicated timetable . . . . . . . . . . . . . . 21 
3.1 .5 Journey . . . . . . . . . . . . . . . . . . 21

3.2 Ferreira's algorithm with $t_{q}$ support $\ldots \ldots \ldots \ldots \ldots \ldots$

3.3 Timetable-Based Algorithms . . . . . . . . . . . . . . . . . . 24

3.3 .1 Pure-Timetable . . . . . . . . . . . . . 26

3.3.2 Hybrid Timetable-Evolving Graph . . . . . . . . . . . . 30

3.3.3 Timetable with Auxiliary Graph . . . . . . . . . . . . . 33

3.4 Journey extraction . . . . . . . . . . . . . . . . . 35

4 Data sets $\quad 36$

4.1 Evolving graph and timetable generation . . . . . . . . . 36

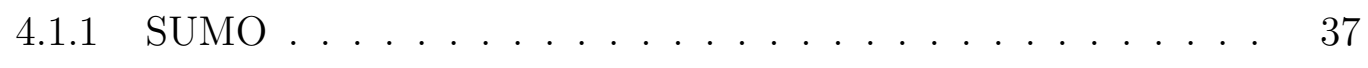

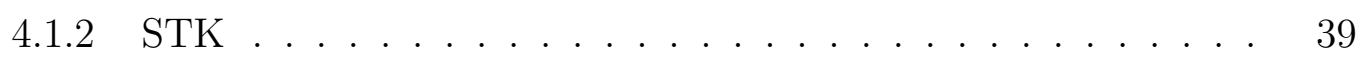

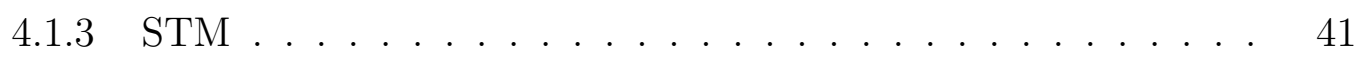

4.2 Characteristics of data sets . . . . . . . . . . . . 42

4.2 .1 Time slots . . . . . . . . . . . . . . . 42

4.2 .2 Number of connections . . . . . . . . . . . . . 4 43

4.2 .3 Presence ratio . . . . . . . . . . . . . . . 43

4.2 .4 Dynamic ratio . . . . . . . . . . . . . . . 47

5 Experimental results $\quad 52$

5.1 Computed journey characteristics analysis $\ldots \ldots \ldots \ldots$

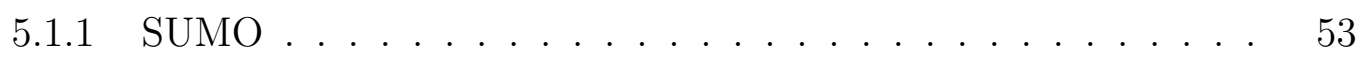

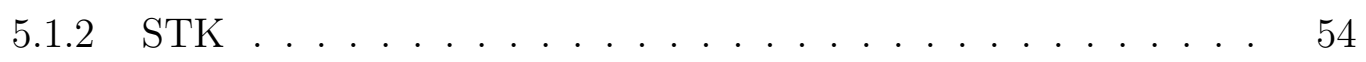

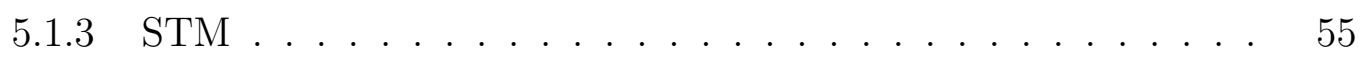

5.2 Performance analysis $\ldots \ldots \ldots \ldots \ldots \ldots \ldots$

5.2 .1 Effect of varying the number of nodes . . . . . . . 56

5.2 .2 Effect of varying the transmission range $\ldots \ldots \ldots$

6 Conclusions $\quad 65$

$\begin{array}{ll}\text { Bibliography } & 66\end{array}$ 


\section{List of Figures}

1 Least cost journeys from $A$ to $D$ at time no earlier than 0 . The number above any node $v$ on the journey is the cost of the specific least cost journey from $A$ to the node $D . \ldots \ldots \ldots$

2 Fixed-schedule Evolving Graph data structure [53]. . . . . . . . . . 17

3 Evolving Graph example. . . . . . . . . . . . . . . . . . 18

4 Evolving Graph data structure example. . . . . . . . . . . . . . 19

$5 \quad \mathcal{C}$ data structure . . . . . . . . . . . . . . . . 20

$6 \quad \mathcal{C}$ data structure example. . . . . . . . . . . . . . . . . . . 21

$7 \quad$ Complexity analysis example. . . . . . . . . . . . . . . 31

8 Road network for SUMO simulation. . . . . . . . . . . . . 37

9 SUMO data parsing. . . . . . . . . . . . . . . . . . . . 38

10 SUMO different density of nodes and transmission ranges. . . . . . 39

11 Satellite STK data parsing. . . . . . . . . . . . . . . . 40

12 STK screenshot. . . . . . . . . . . . . . . . . . . . 40

13 STM data parsing. . . . . . . . . . . . . . . . . 41

14 SUMO number of connections. . . . . . . . . . . . . . . 44

15 Number of connections in SUMO data set as the transmission range reaches its limit. . . . . . . . . . . . . . . . . . 4 44

16 STK number of connections. . . . . . . . . . . . . . 45

17 STM number of connections. . . . . . . . . . . . 45

18 Presence ratio example. . . . . . . . . . . . . . . . 46

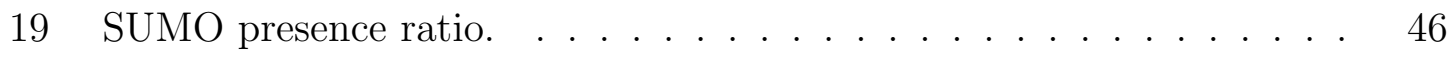

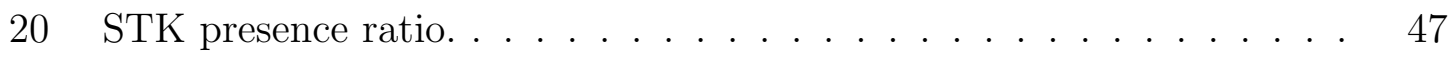

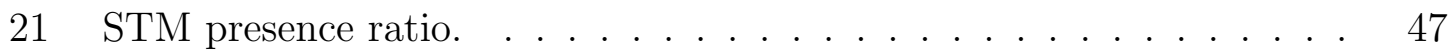

22 Schedule with intervals switching between active and non-active. . . . 48

23 Schedule with only one interval. . . . . . . . . . . . . . 48 
24 Schedule with one interval with three consecutive time steps. . . . . 48

25 SUMO dynamic ratio. . . . . . . . . . . . . . . . . . 49

26 SUMO comparison between presence ratio and dynamic ratio. . . . . 50

27 STK dynamic ratio. . . . . . . . . . . . . . . . . . . 51

28 STM dynamic ratio. . . . . . . . . . . . . . . . . 51

29 SUMO varying the number of nodes. . . . . . . . . . . . . 53

30 SUMO varying the transmission range. . . . . . . . . . . 53

31 STK varying the number of nodes. . . . . . . . . . . . 54

32 STK varying the transission range. . . . . . . . . . . . . 54

33 STM varying the transmission range. . . . . . . . . . . 55

34 SUMO number of hops varying the number of nodes. . . . . . . . 56

35 SUMO running time varying the number of nodes. . . . . . . . . 57

36 STK number of hops varying the number of nodes. . . . . . . . 58

37 STK running time varying the number of nodes. . . . . . . . . . 59

38 SUMO number of hops varying the transmission range. . . . . . . . 60

39 SUMO running time varying the transmission range. . . . . . . . 61

40 STK number of hops varying the transmission range. . . . . . . . . 62

41 STK running time varying the transmission range. . . . . . . . . 63

42 STM number of hops. . . . . . . . . . . . . . . . . . . . 64

43 STM running time varying the transmission range. . . . . . . . 64 


\section{List of Tables}

1 Time slots. . . . . . . . . . . . . . . . . 43 


\section{Chapter 1}

\section{Introduction}

\subsection{Motivation}

Advances in wireless technologies, vehicular networking, and automobiles have made it possible to conceive of heterogeneous vehicular networks comprising vehicles, roadside units, as well as pedestrians. Intelligent transportation systems (ITS) consider vehicles as network components responsible for sending, receiving and routing packets within the vehicular ad hoc network (VANET). Each vehicle is equipped with a radio antenna called an on-board unit or OBU. In such network, vehicle connectivity is classified as Vehicle-to-Vehicle (V2V), Vehicle-to-Infrastructure (V2I) and Vehicle-to-Pedestrian (V2P). V2V involves data exchange between two or more vehicles while V2I considers the interaction between vehicles and the road environment. $\mathrm{V} 2 \mathrm{P}$ includes pedestrians as potential network members [4]. One emerging type of connectivity is V2X known as Vehicle-to-Everything which also includes communication with IT networks and data centers [31].

Among the applications that VANETs support are urban sensing, interactive entertainment, comfort, efficiency and safety [13]. Due to its potential, Transport Canada created the Advance Connectivity and Automation in the Transportation System (ACATS) program whose aim is to prepare Canadian roads for connected and automated vehicle deployments to support V2V, V2I and V2X communication [4]. For instance, Montréal is currently working with EasyMile [17] and Transdev [27] to implement an automated shuttle near the Olympic Stadium area to reduce the number of on-road collisions [4]. Another V2V application developed in collaboration 
with many truck companies and supported by U.S. Department of Transportation and the European Union is the SARTRE project which estimates to reduce emissions and increase safety and comfort. It forms road trains on highways where the leading car takes the responsibility for the entire road train and every car mimics its actions. This allows the drivers to focus their attention on other things [39].

In vehicular ad hoc networks, $\mathrm{V} 2 \mathrm{~V}$ communication is challenging because communication links are transient, causing the network topology to keep changing. There is therefore no permanent route between any two nodes in the network. Indeed at any given time step, it may not be possible to guarantee a route between any given source and destination. The situation could be even worse: for a given source-destination pair, it may be that there is no time at which there is a route between them. However, by storing packets at intermediate nodes, it may be possible to deliver the packet eventually to the destination. It is for this reason that such networks have been variously called delay or disruption-tolerant, or intermittently-connected networks over the last couple of decades.

This thesis considers a particular type of vehicular network: Fixed Schedule Dynamic Network (FSDN) also known as Time Varying Communication Network (TVCN) [29]. Such a network has a fixed set of nodes, which move in the given terrain using a pre-determined schedule and trajectory, i.e. route/path in the terrain. As two nodes in the network move within transmission range, a communication link or connection is created. As the nodes move away from each other, the connection is broken, and may or may not re-appear, depending on the trajectories of the nodes. Indeed a single pair of nodes may be part of many different connections at different times; all these connections are said to correspond to the same edge. Since the trajectories of the nodes are known, the network is dynamic but in a predictable manner; for each connection, we know in advance (roughly) when it will start and when it will end.

Examples of FSDN networks include networks created by public transport buses, Low Earth orbiting satellites (LEO), etc. For instance, Unmanned Aerial Vehicles (UAVs) have a predictable circular flight pattern, so they are able to compute routes withing the UAV network at any time [48] [3] [40]. They can support applications such as tactical military applications, humanitarian aid missions, drug blockage enforcement. DakNet [42] provides internet access in developing nations; some links in 
the Daknet network can be predicted due to the mobility patterns of public transportation vehicles [28]. LEO satellite networks are classified as FSDN as well since the position of each satellite is deterministic due to its trajectory around the earth and links between satellites can be estimated [19]. Another example where the presence of the links can be estimated is Wireless Sensor Networks (WSNs) where connections between sensors appear and disappear based on a sleep schedule due to their energy constraints [20].

FSDNs can be represented by dynamic graphs, which have received a lot of attention recently [29]. There are many models of such graphs that have been studied, and we describe these in Chapter 2. For now, we restrict ourselves to saying that a dynamic graph $\mathcal{G}$ is defined as a pair $\left(G, \mathcal{S}_{G}\right)$ where $G=(V, E)$ is a graph with a set of nodes $V$ and a set of edges $E$ and $\mathcal{S}_{G}=G_{0}, G_{1}, \ldots, G_{k}$ is an ordered sequence of sub graphs $G_{i}$. Each $G_{i}$ has a set of nodes $V_{i}$ and a set of edges $E_{i}$ active in the time interval $\left[t_{i}, t_{i}+1[\right.$. Two nodes are adjacent in $\mathcal{G}$ if they are adjacent in any $G_{i}$. A common representation for such graphs is to use the base graph $G$, and for each edge, store a sorted list of distinct and maximal time intervals in which it is alive. A temporal path in a dynamic graph is a sequence of $\left(v_{i}, t_{i}\right)$, where each $v_{i} \in V$, so that the pair $\left(v_{i}, v_{i+1}\right)$ is connected at time $t_{i}$, and furthermore, the $t_{i}$ 's form a non-decreasing sequence.

\subsection{Problem definition}

In this thesis, we study the problem of one-to-one routing in an FSDN represented by an evolving graph $\mathcal{G}$. In particular, at a given time, given a source node $s$ and destination node $t$ in $\mathcal{G}$, and a query time $t_{q}$, we wish to compute a temporal path in the network that minimizes the arrival time at the destination. Such a path is sometimes called a foremost journey in the network.

We assume that time is slotted into discrete time slots, and that the mobility of the nodes/vehicles/satellites is such that the transmission time for a packet is negligible compared to the minimum duration of a connection. Note that we are not guaranteed a source-destination path in the network at any given time; so while several hops in the network may be traversed almost instantaneously, there may be time intervals where it is not possible to make progress towards the destination. 
This problem was introduced in [21], and the authors gave an algorithm based on Dijkstra's shortest path algorithm, that used an evolving graph data structure to represent the network. Their algorithm was shown to take $\mathcal{O}(|E|(\log I+\log |V|))$ time where $E$ is the set of edges and $I$ is the maximum number of time intervals in which an edge is present.

\subsection{Our results}

We give a new timetable-based approach for the foremost journey computation problem, and describe three variants of this approach. The timetable $\mathcal{T}$ stores the connections that appear in the FSDN in order of their start time.

- Our first algorithm, called Pure-Timetable takes time $\mathcal{O}(|V||E|+|\mathcal{T}|)$ and space $\mathcal{O}(|\mathcal{T}|)$. In addition to the timetable of connections, we use an auxiliary timetable to save connections that were not useful when they started but may prove to be useful later.

- Our second algorithm is a hybrid approach that uses both the timetable of connections, and the evolving graph data structure of $[21]$ and takes $\mathcal{O}(|E| \log I+$ $|\mathcal{T}|+|V|)$ time and space $\mathcal{O}(|\mathcal{T}|)$.

- Our final variant called Timetable with Auxiliary Graph uses both the timetable of connections, and an auxiliary graph data structure created and maintained during processing of the timetable $\mathcal{T}$. This last variant finds the route between any source-destination pair in $\mathcal{O}(|E|+|\mathcal{T}|+|V|)$ time and space $\mathcal{O}(|\mathcal{T}|)$.

Note that $|\mathcal{T}|=\mathcal{O}(|E| I)$. It is clear from the above that Hybrid TimetableEvolving Graph and Timetable with Auxiliary Graph improve over the classic algorithm of Ferreira [21] in some situations. We also do extensive simulations of our algorithms, using random data sets, as well as actual data sets derived from the Société de transport de Montréal and satellite positions that validate the superior performance of our algorithms. 


\subsection{Organization}

In Chapter 2, we review the literature concerning dynamic graph models, journey computation and previous algorithms proposed for our problem and related problems. Chapter 3 defines the data structures and the algorithms that we propose describing each timetable-based variant along with Ferreira's algorithm [21]. In Chapter 4 we detail the procedure to obtain the timetables and evolving graphs which are the inputs for our experiments; then, we provide some metrics to characterize each data set. Finally, Chapter 5 discusses the performance of each algorithm and explains the attributes of the computed journeys; e.g., number of hops, journey duration. We conclude with some directions for future research in Chapter 6. 


\section{Chapter 2}

\section{Related work}

This chapter reviews the literature related to the scope of this work. In Section 2.1, we review the mathematical models that are employed to represent dynamic graphs. Then in Section 2.2, we discuss the previously proposed algorithms related to journey calculation in communication networks. Finally, we describe an algorithm designed to find foremost journeys for passengers in transportation networks, called Connection Scan Algorithm in Section 2.3.

\subsection{Dynamic graph models}

In the literature, there are two types of work on dynamic graph. In the first type, researchers have considered how to support operations that modify the initial state of the graph such as edge insertion, edge deletion, node insertion and node deletion, as soon as they happen. In this work, a dynamic graph is also known as a streaming graph [5] and is modeled with data structures such as STINGER [18], cuSTINGER [23], EvoGraph [47], AIMS [51], Hornet [7]. As an example, Green's algorithm [22] is a well-known algorithm that updates the value of betweenness centrality ${ }^{1}$ metric [24] [49] as soon as a change in the graph topology occurs.

The scope of this work focuses on the second type of dynamic graph where the presence of an edge depends on a given time within the timespan of the graph, and can be known or predicted in advance. For instance, Low Earth Orbiting (LEO)

\footnotetext{
${ }^{1}$ The betweenness centrality of a node $v$ is defined as $C_{B}(v)=\sum_{s \neq z \neq v} \sigma_{s z}(v) / \sigma_{s z}$ where $\sigma_{s z}$ is the number of shortest paths between node $s$ and node $z$ while $\sigma_{s z}(v)$ is the number of shortest paths between node $s$ and node $z$ that pass through node $v$ [24].
} 
satellite systems have links in different orbital planes where each endpoint belongs to a different cluster of satellites. At some point, they are out of range due to their relative movement. Such networks are called Fixed Schedule Dynamic Networks (FSDN); the changes to the topology of such a network and can be predicted ahead of time [21].

There have been efforts to formulate a dynamic graph model that successfully captures the dynamic behavior of a network. However, there is a lack of consensus among those models since the approach to formulate each is different. According to [56], dynamic graph models can be grouped in four main categories: sequence of snapshots, whole graph, log file and distributed graph over servers. The following subsections have a brief overview of each dynamic graph model.

\subsubsection{Snapshots}

Models based on a sequence of snapshots have a static graph per time step. For example, the authors in [54], propose algorithms to capture the Most Frequently Changing Component (MFCC) given a sequence of snapshots. There is a notion introduced in [43] for sequence of static graphs called Evolving Graph Sequence (EGS). They formulate a framework called FVF for efficient query processing in EGSs. Also, a Dynamic Behavioral Mixed-Membership Model (DBMM) is studied in [45] to identify the roles that nodes play in social networks. Normally, every snapshot in the sequence is encoded as a triple $\left(V_{i}, E_{i}, t_{i}\right)$ where the set of nodes $V_{i}$ and the set of edges $E_{i}$ are present at $t_{i}$ time step [41].

The downside of this kind of model is that it requires a lot of space to store each snapshot. However, to overcome this problem and improve its memory performance some techniques have been studied. For instance, the authors in [44] improve the FVF framework including a preprocessing step that consist of identifying similar snapshots and group them into clusters, then two representative graphs are extracted. Similar approaches are proposed in [45] and [57]. Authors in [45] define a method to cluster snapshots that have similar query frequency and [57] formulates a procedure that chooses snapshots with lowest variance. 


\subsubsection{Whole graph}

The idea of the whole graph model is to represent a dynamic graph as one single graph. The key aspect is the introduction of time intervals where nodes or edges are considered present (sometimes termed valid, alive, or active). This can be represented by a presence function [9] for both nodes and edges, which given a specific time instant, outputs if they are alive or not.

The formalization of this whole graph approach is given in [21] and [9], also known as FSDN and Time-Varying Graphs (TVGs), respectively. Depending on the application the model may vary but the approach is the same. For example, the authors in [32] use labels for each edge to denote the time interval when they are alive. Another model similar to TVG called Temporally Evolving Graph (TEG) is described in [26] and [25]. Authors in [35] propose a model called Space-Time Varying Graph (STVG); they define adjacency between nodes based on places and events across time.

The authors of [50] propose what they term a unifying model for representing time-varying graphs. Basically, they claim that their model can unify both whole graph and snapshot models. They represent edges as a quadruple $\left(u, t_{a}, v, t_{b}\right): u$ and $v$ are the source and target nodes, $t_{a}$ and $t_{b}$ represent the time interval. Then, they identify four types of edges based on their temporal characteristics. Spatial edges connect two different nodes at the same time $t_{a}=t_{b}$ and $u \neq v$. Temporal edges connect the same node but at different time $t_{a} \neq t_{b}$ and $u=v$. Mixed edges link two different nodes at different time $t_{a} \neq t_{b}$ and $u \neq v$ and Spatial-Temporal edges link the same node at the time $t_{a}=t_{b}$ and $u=v$. Afterwards, they prove that any dynamic graph that is modeled with snapshots or whole graph and has those type of edges can be represented with their unifying model.

\subsubsection{Log file}

Log file models are similar to snapshot models but they follow a materialization procedure [56]. This procedure stores snapshots along with delta log files to store the changes in the topology. It saves memory space since it is not required to store the whole snapshot but it requires an additional processing power to construct the snapshot using the log files.

There are three strategies to materialize snapshots: Time-based, Operation-based and Similarity-based [56] [52]. Time-based materialization fixes the elapsed time 
between two materialized snapshots. Operation-based materialization establishes a constant number of events to materialize a snapshot. Similarity-based materialization keeps a threshold between two snapshots to be considered similar.

The authors of [52] call their model snapshot plus log which is a similarity-based model since they cluster snapshots according to the distribution of historical queries. They assert that the other two materialization strategies may result in a storage or processing power degradation. TGraph [38], ChronoGraph [8] and Immortalgraph [36] are examples of time-based models while DeltaGraph [30] considers the number of events as the main criteria to materialize a snapshot.

\subsubsection{Distributed graph over servers}

The models described above are organized by time: the dynamic behavior is indexed by the time it takes place. The models that belong to the category of distributed graph over servers are called entity-centric as their formulation focuses on nodes and their history during the timespan of the dynamic graph [33]. Nodes are organized in subsets which are handled by a set of servers. Each server maintains the history of a subset of nodes [56]. For instance, HiNode [33] saves the history of each node using Interval Trees and B-Trees. The authors of [34] introduce materialization techniques and parallel complex graph data processing to reduce the memory usage while the model in [55] involves delta log files in its storage in order to reduce the update time.

\subsection{Journey computation}

Paths are to static graphs as journeys are to dynamic graphs. Journeys incorporate the time domain to travel between a source node and a destination node within the network. In static graphs, the shortest path between two nodes is the one that has the minimum cost in terms of number of hops, delay, etc. For dynamic graphs, a journey is undertaken at a given time $t$. There are three commonly studied notions of least cost [53].

- Shortest journey: It ignores the time domain and among all possible journeys, it has the least number of hops between the source node and the destination node, starting from the source node at time no earlier than $t$. 
- Fastest journey: It has the minimum difference between arrival time and departure time, starting from the source node no earlier than time $t$.

- Foremost journey: It has the earliest arrival time at the destination, starting from the source node at time no earlier than $t$.

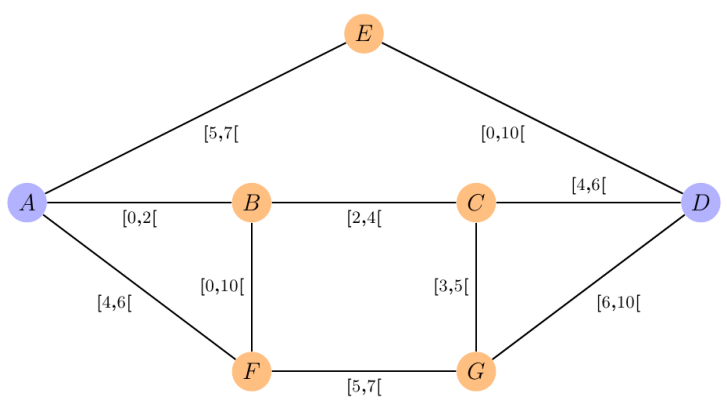

(a) Dynamic graph.

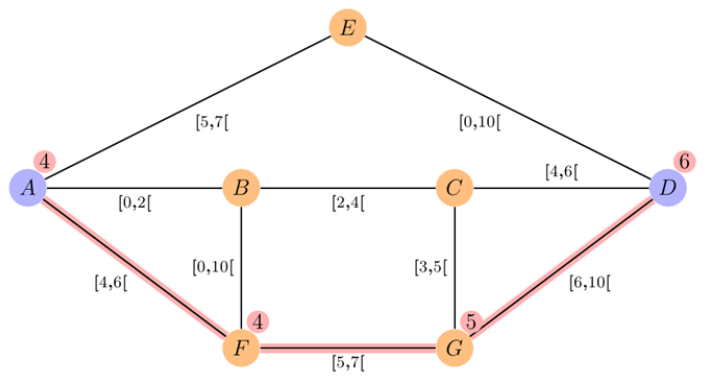

(c) Fastest journey.

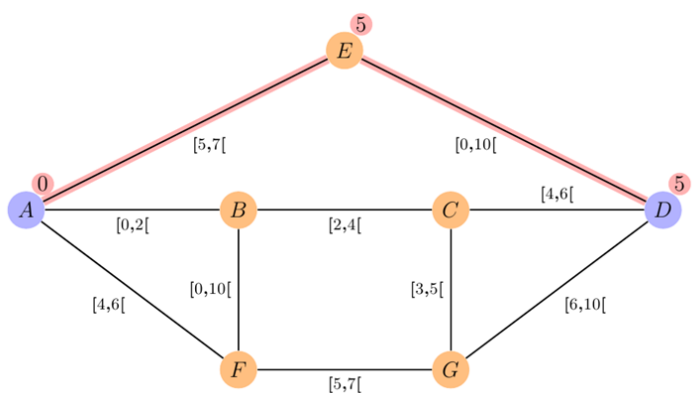

(b) Shortest journey.

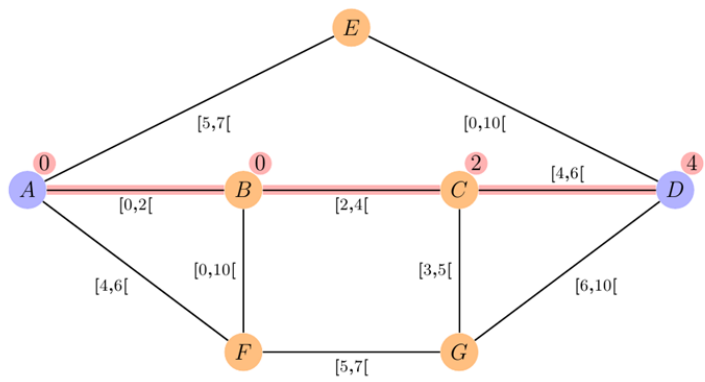

(d) Foremost journey.

Figure 1: Least cost journeys from $A$ to $D$ at time no earlier than 0 . The number above any node $v$ on the journey is the cost of the specific least cost journey from $A$ to the node $D$.

Figure 1 shows a dynamic graph and illustrates the three types of journeys described above. For example, for the dynamic graph shown in Figure 1a, the Figures 1b, 1c, 1d, show the shortest, fastest, and foremost journeys respectively.

We describe some algorithms to compute journeys. The first one and the most expensive one in terms of running time is Matrix Multiplication Algorithm [58] [28]. It uses adjacency matrices for each time step and multiplies them to calculate shortest, fastest and foremost journeys. The authors in [58] show that their approach takes $\mathcal{O}\left(|V|^{4}\right)$ time $^{2}$ per time step to compute shortest journeys. The second one is a family

\footnotetext{
${ }^{2}$ Using faster matrix multiplication algorithms [6], it may be possible to improve the complexity of their approach.
} 
of algorithms that are extensions or modifications of original Dijkstra's algorithm [16]. Finally, the last algorithm, proposed in the context of finding journeys for passengers in scheduled train or subway transportation networks, is Connection Scan Algorithm (CSA) [15]. We describe below further details about Dijkstra's algorithm extensions.

\subsubsection{Dijkstra's algorithms extensions}

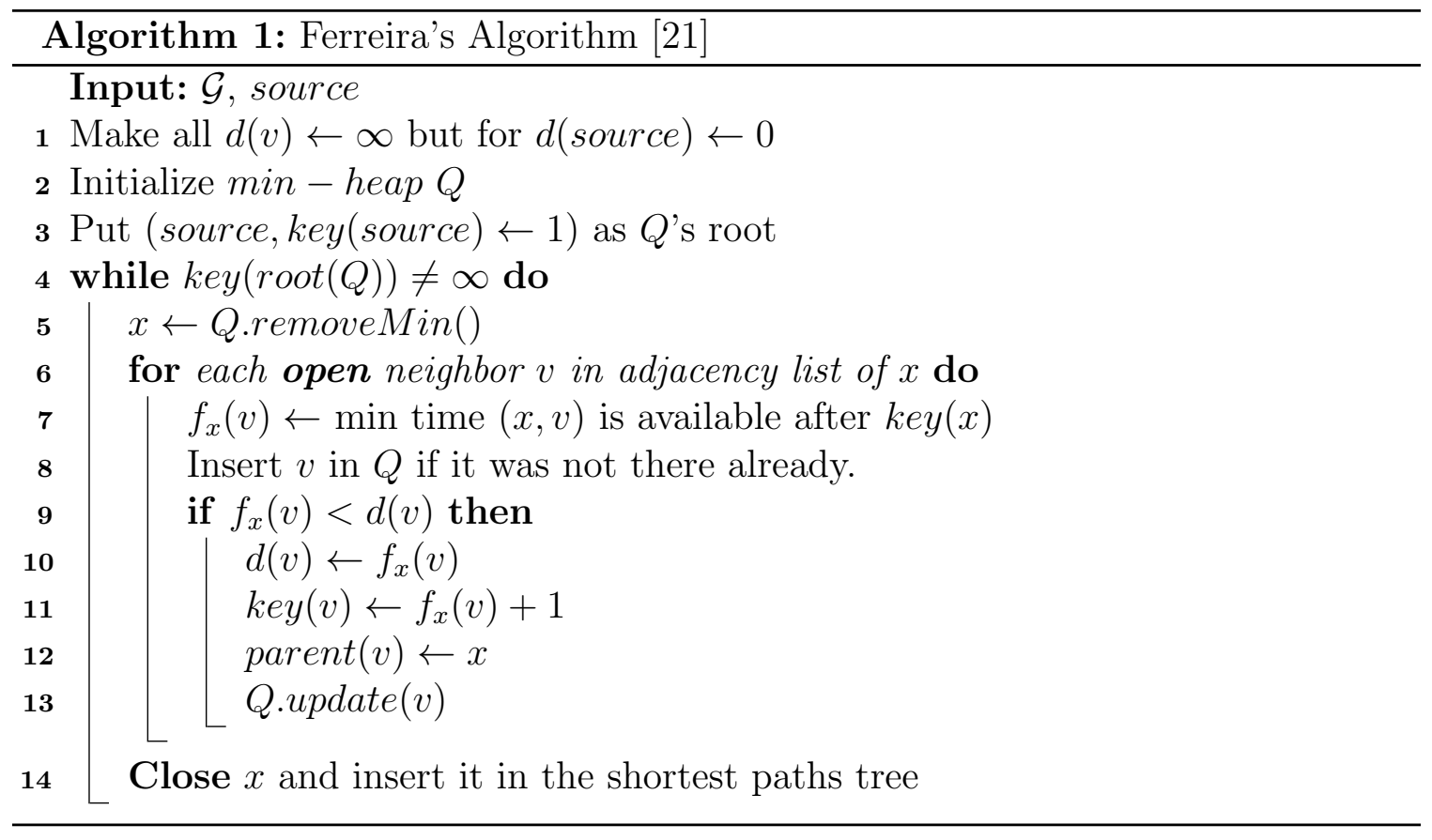

The first author who introduced an algorithm to compute foremost journeys in Evolving Graphs is Ferreira [21]. This is also our main reference to compare the performance of our proposed algorithms, so we give the pseudocode in Algorithm 1 and describe it in detail here. This algorithm has an evolving graph $\mathcal{G}$ and source node as inputs. The following chapter defines $\mathcal{G}$ in detail, but as we can see the algorithm uses a priority queue $Q$. Every node $v$ in $\mathcal{G}$ has a $d(v)$ attribute which contains the estimate of the earliest time $v$ can be reached. This value is initialized to zero for the source node and infinity for all other nodes. A node is closed if the estimate is the final correct value, and it is open otherwise. As in Dijkstra's algorithm, we remove the node $x$ with least $d$ value from the priority queue, and relax all its neighbors, after which $x$ can be closed. It is the relaxation step that differs from Dijkstra. For each open neighbor $v$ of $x$, we need to compute $f_{x}(v)$, the next time 
there is a connection between $x$ and $v$. This calculation involves looking into the schedule of the edge $(x, v)$ to know when $v$ can be reached, and can be performed in $\mathcal{O}(\log I)$ time using binary search, where $I$ is the maximum number of intervals in which an edge is active. Line 11 of Algorithm 1 sets $d(v)$ to be $f_{x}(v)+1$. This is because the author considers a delay of one unit of time to forward a packet to its neighbor. One potential bottleneck is Q.update $(v)$, if the value of $v$ changes its priority has to be updated, which takes $\mathcal{O}(\log |V|)$ time.

As proved in [21] and outlined above, for each edge adjacent to a closed vertex, Algorithm 1 performs $\mathcal{O}(\log I+\log |V|)$, where $I$ is the max number of time intervals in which an edge is present. $|V|$ is the number of nodes. Thus the algorithm takes time $\mathcal{O}(|E|(\log I+\log |V|))$.

Algorithms for shortest and fastest journey computation were introduced in [53]. These algorithms are also modifications or extensions of Dijkstra's algorithm [16]. Some speed-up techniques have been introduced to find shortest journeys faster. For example, the author in [25] extends bidirectional Dijkstra's algorithm and Landmarkbased $\mathrm{A}^{*}$ to compute shortest journeys in TEG's. Another speed-up technique incorporated in [25] is Contraction Hierarchies (CH). Basically, $\mathrm{CH}$ allows to add shortcuts into the original TEG so the algorithm is able to bypass nodes that are not important during shortest journey calculation. The downside of $\mathrm{CH}$ incorporation is that it adds a huge pre-processing overhead.

\subsection{Connection scan algorithm}

Connection Scan Algorithm (CSA) [15] was designed as a route planning algorithm for public transit systems, involving a combination of trains, buses, trams, for example. Users of such algorithms may not only want to find the earliest arrival time to reach a certain destination, but may also have other criteria, such as minimizing the number of hops. Train and bus stops are connected by footpaths which incur a certain delay. CSA outputs a sequence of footpaths-vehicles that a traveler has to follow in order to reach a desired destination from a given source position, that optimizes the given criteria.

In CSA, the public transportation system is modeled as a timetable which is a quadruple of stops, connections, trips, and footpaths $(\mathcal{S}, \mathcal{C}, \mathcal{T}, \mathcal{F})$, respectively. The 


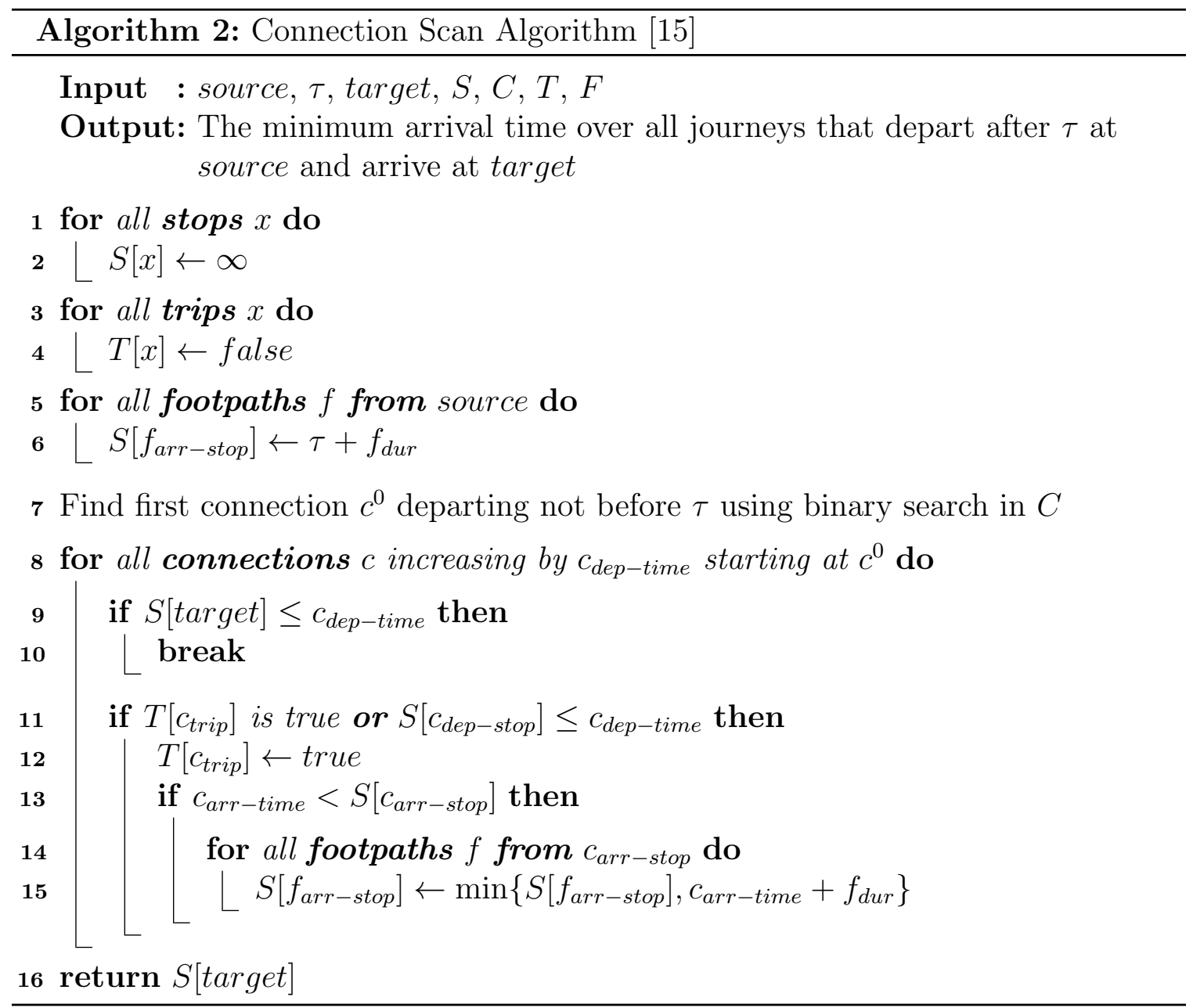

footpath graph $\mathcal{F}$ has the stops listed in $\mathcal{S}$ as nodes and the edges are the footpaths that the traveler can walk. The weight for each edge $f_{d u r}$ is the time that it takes to walk from one stop to another. Note that each stop has a self-loop whose (non-zero) weight is the time taken to transfer at the stop. $\mathcal{T}$ is the set of trips which are scheduled vehicles. Connections in $\mathcal{C}$ describe how vehicles drive; they are modeled as departure stop, arrival stop, departure time and arrival time $\left(c_{\text {dep }}, c_{\text {arr }}, c_{\text {dep-time }}, c_{\text {arr-time }}\right)$, respectively. A trip is described as a sequence of connections driven by the same physical vehicle.

Algorithm 2 shows the earliest arrival Connection Scan Algorithm variant [15]. The inputs are the quadruple timetable, a source stop, a target stop and the departure time $\tau$. This algorithm assumes that connections are ordered by departure time and the footpath graph is stored as adjacency lists. If we contrast this algorithm 
with Dijkstra's algorithms extensions then we see that there is no priority queue to keep a tentative arrival time. Instead, it keeps two arrays; $\mathcal{S}$ array maintains the earliest tentative arrival time for each stop and $\mathcal{T}$ array keeps track which trips have been boarded. One connection $c$ is reachable if the traveler is at the departure stop $c_{d e p-s t o p}$ before departure time $c_{\text {dep-time }}$ or the trip flag $\mathcal{T}\left[c_{\text {trip }}\right]$ is set. This flag indicates that a connection that belongs to the same trip was boarded before and the traveler can remain seated. Once the algorithm finds a reachable $c$ connection; the algorithm modifies the tentative arrival time at the stops reachable by foot from the arrival stop $c_{\text {arr-stop }}$. This Connection Scan Algorithm variant already includes three optimizations. One called stopping criterion in line 9 which ends the execution after finding a connection such that its departure time is greater than the tentative arrival time at target. The second one known as starting criterion in line 7 that finds a connection $c^{0}$ whose departure is not before $\tau$ using binary search. The last optimization limits the iteration of outgoing footpaths when the time at $\mathcal{S}\left[c_{\text {arr-stop }}\right]$ is greater than $c_{\text {arr-time }}$ in line 13 .

\section{CSA journey extraction}

Algorithm 2 only outputs the earliest arrival time at the target stop but there is no information about the journey. A journey in [15] is an alternating sequence of legs and footpaths that indicates the passenger how to travel within the network; it must start and end with a footpath. In this context, a leg is a pair of connections that belong to the same trip; the first one is the enter connection $l_{\text {enter }}$ and the latter is the exit connection $l_{\text {exit }}$. Logically, $l_{\text {enter }}$ must appear before $l_{\text {exit }}$ in the timetable. There is a footpath between legs, this leg transition is called transfer; it must follow the transfer model that the authors in [15] propose. Basically, the transfer model tells the feasibility of boarding a departing connection $c^{\prime}$ at stop $c_{\text {dep-stop }}^{\prime}$ coming from a connection $c$ arriving at stop $c_{\text {arr-stop. }}$ In other words, it is possible to board a departing connection that belongs to another trip as long as the passenger is at the departure stop before the departure time. Note that, loops in this model allow the passenger to transfer at the same stop. They call the duration $f_{d u r}$ of the loop as change time and every other footpath that is not a loop is called interstop footpath [15].

They propose two ways to extract a journey. The first one uses something that 
the authors in [15] call journey pointers which requires an extension of the current data structures. The second one extracts the journey without journey pointers but needs more complex tasks and data structures. Depending on the scenario, one is better than the other in terms of running time. If only one journey from a source stop towards a target stop is needed then the approach without journey pointers is faster. But, if many journeys from one source stop towards many target stops are needed then the journey pointers approach is faster and easiest to implement.

Journey pointer calculation requires a modification of $\mathcal{T}$ array and an addition of a new $\mathcal{J}$ array that holds the journey pointers for each stop. Instead of saving a boolean flag in $\mathcal{T}$ for each trip, it holds the ID of the first reached connection for each trip. $\mathcal{J}$ array keeps triples that consist of the exit connection, the final footpath and the enter connection. Algorithm 2 shows that at line 15 the journey pointer can be easily calculated since the exit connection and the final footpath are right there. But, there is no information about the enter connection. So, to complete the triple the algorithm queries the modified $\mathcal{T}$ array to retrieve the ID of the enter connection. Once the algorithm finishes its execution; it is enough to follow the pointers and back track the journey starting at the target stop until the source stop is found.

Journey extraction without journey pointers requires two adjacency matrices. One matches the trips IDs with the journey IDs. So, the algorithm is able to iterate the connections that belong to the same trip fast. The second adjacency matrix encodes the stop ID with a sorted list which contains the IDs of the arriving connections at that stop. The algorithm uses a subroutine to calculate the enter connection, the exit connection and the footpath to form the triple. As described earlier, the enter connection is hard to calculate. So, to overcome this problem this approach builds a set of candidate enter connections for each exit connection. Then, the list of connections that belong to the same trip of a candidate connection is retrieved. A valid enter connection is the $x$ connection within that list such that the time at the departure stop is less than or equal to the $x$ departure time $S\left[x_{d e p-s t o p}\right] \leq x_{d e p-t i m e}$. Finally, the candidate sets are pruned and the journey is extracted. 


\section{Chapter 3}

\section{Timetable-Based journey computation}

In this chapter, we describe a timetable-based approach to computing foremost journeys in FSDN, that is inspired by the Connection Scan Algorithm described in the previous chapter. First, in Section 3.1 we give some basic definitions and describe the data structures used, starting with the Evolving Graph $\mathcal{G}$ definition introduced in [21]. Then, we specify our definitions of connection, timetable, duplicated timetable and journey.

In Section 3.2 we describe a slight modification in the original Ferreira's algorithm [21] to support the query time $t_{q}$. Then Section 3.3, presents what we call Timetablebased Algorithms. So, we propose the following algorithms: Hybrid TimetableEvolving Graph, Pure-Timetable, Timetable with Auxiliary Graph. The name of each algorithm has to do with the approach to handle non-relaxed connections. Finally, in Section 3.4 a simple journey extraction algorithm is presented.

\subsection{Preliminaries}

\subsubsection{Evolving graph}

The authors in [53] define $\mathcal{G}$ as a system $\mathcal{G}=\left(G, \mathcal{S}_{G}\right)$ where $G=(V, E)$ is a graph with a set of nodes $V$ and a set of edges $E$ and $\mathcal{S}_{G}=G_{0}, G_{1}, \ldots, G_{s}$ is an ordered sequence of subgraphs $G_{i}$ of $G$. Each $G_{i}$ has a set of nodes $V_{i}$ and a set of edges $E_{i}$ running in the interval $\left[t_{i}, t_{i}+1[\right.$. Two nodes are adjacent in $\mathcal{G}$ if they are adjacent 
in any $G_{i}$.

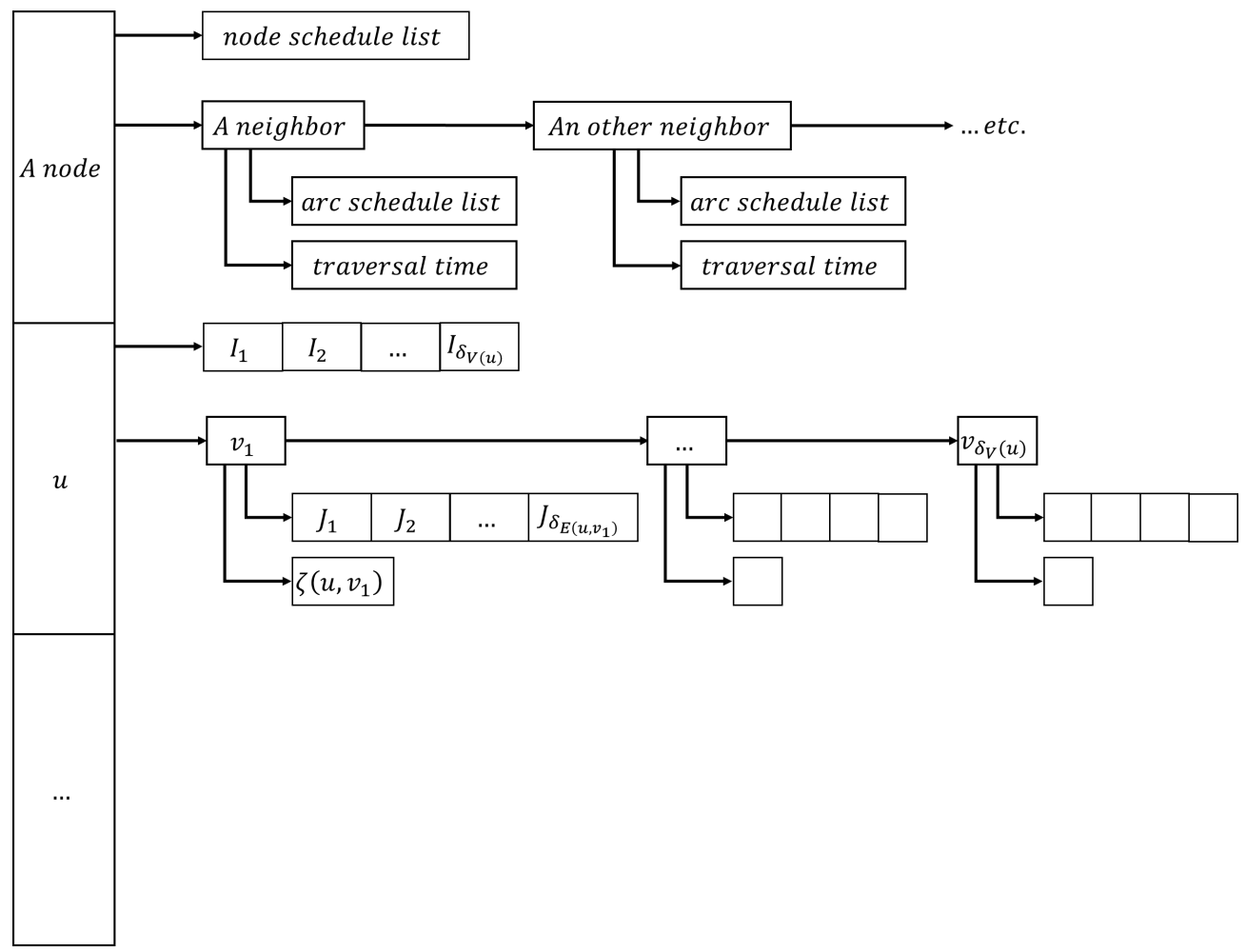

Figure 2: Fixed-schedule Evolving Graph data structure [53].

Alternatively, $\mathcal{G}$ can be defined as $\mathcal{G}=\left(V_{\mathcal{G}}, E_{\mathcal{G}}\right)$ as Equations 1 and 2 show. So, each edge $e$ in $E_{\mathcal{G}}$ has a set of ordered intervals $P_{E}(e)$ defining the edge schedule which contains the time steps when $e$ is present. In addition, each node $v$ in $V_{\mathcal{G}}$ has its own ordered schedule $P_{V}(v)$ indicating the time steps when $v$ is present in $\mathcal{G}$ [53]. Furthermore, the authors provide some notions of node, edge and evolving graph activity: the activity of a vertex $v$ as $\delta_{V}(v)=\left|P_{V}(v)\right|$, the activity of an edge $e$ as $\delta_{E}(e)=\left|P_{E}(e)\right|$, the node activity of an evolving graph as $\delta_{V}=\max \left\{\delta_{V}(v), v \in V_{\mathcal{G}}\right\}$, the edge activity of an evolving graph as $\delta_{E}=\max \left\{\delta_{E}(e), e \in E_{\mathcal{G}}\right\}$ and finally the activity of an evolving graph as $\delta=\max \left(\delta_{V}, \delta_{E}\right)$.

$$
\begin{gathered}
E_{\mathcal{G}}=\bigcup_{i=0}^{j} E_{i}: j \leq s \therefore M=\left|E_{\mathcal{G}}\right| \leq|E|=m \\
V_{\mathcal{G}}=\bigcup_{i=0}^{j} V_{i}: j \leq s \therefore N=\left|V_{\mathcal{G}}\right| \leq|V|=n
\end{gathered}
$$




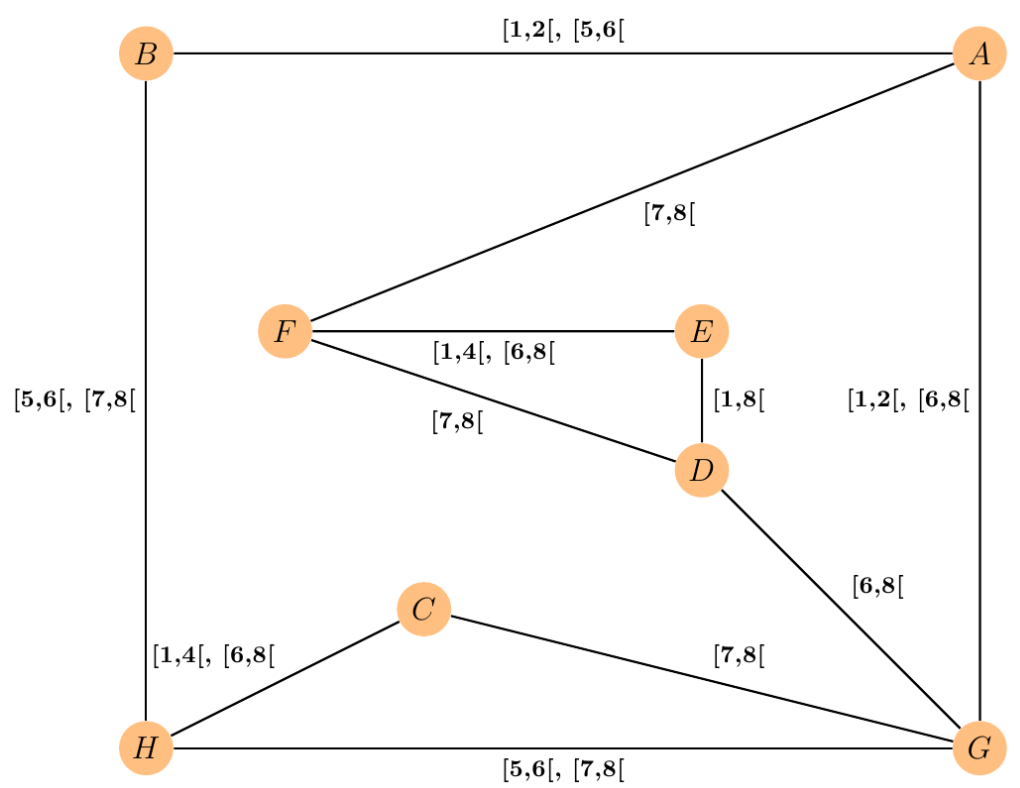

Figure 3: Evolving Graph example.

Similar to weighted static graphs, $\mathcal{G}$ can be weighted too. So, there is a cost to traverse an edge. For instance, the cost can be represented as physical distance, power, hops, delay, etc. If the weight or cost of edge traversal belongs to the time domain then $\mathcal{G}$ defines a function $\zeta(u, v)$ which models the time that it takes to traverse from node $u$ to node $v$.

Ferrerira's algorithm is first described in [21] and the data structure used $\mathcal{G}$ is described in detail in [53]. As shown in Figure 2, $\mathcal{G}$ is coded as a linked adjacency list. To indicate when an edge is present and how long takes to traverse it, each neighbor $v$ has its own sorted edge schedule $P_{E}(e)$ given as time steps intervals and $\zeta(u, v)$ attached to it. Also, each node $u$ in $\mathcal{G}$ has a sorted list of intervals $P_{V}(v)$ to point the time steps when it appears. In other words, $\mathcal{G}$ 's model considers that there may be time steps when a node disappears [21]. The authors prove that the size of $\mathcal{G}$ in the worst case is $\mathcal{O}\left(M+M * \delta_{E}+N * \delta_{V}\right)=\mathcal{O}(M * \delta)$.

For the scope of this work we make three considerations regarding $\mathcal{G}$ 's coding and interpretation. First, we consider that every $G_{i}$ in $\mathcal{S}_{G}$ has the same set of nodes $V_{0}=V_{1}=\cdots=V_{s}$. Therefore the node schedule $P_{V}(v)$ is not needed since every node is always present and never disappears. Our implementation of $\mathcal{G}$ therefore requires only the edge schedule $P_{E}(e)$ as time step intervals for every neighbor. For instance, Figure 3 shows an example of an Evolving Graph. The label in each edge is 
the schedule with the intervals that indicates when the edge is present or alive. Also, Figure 4 shows how Figure 3 would be coded with our considerations for the Evolving Graph data structure.

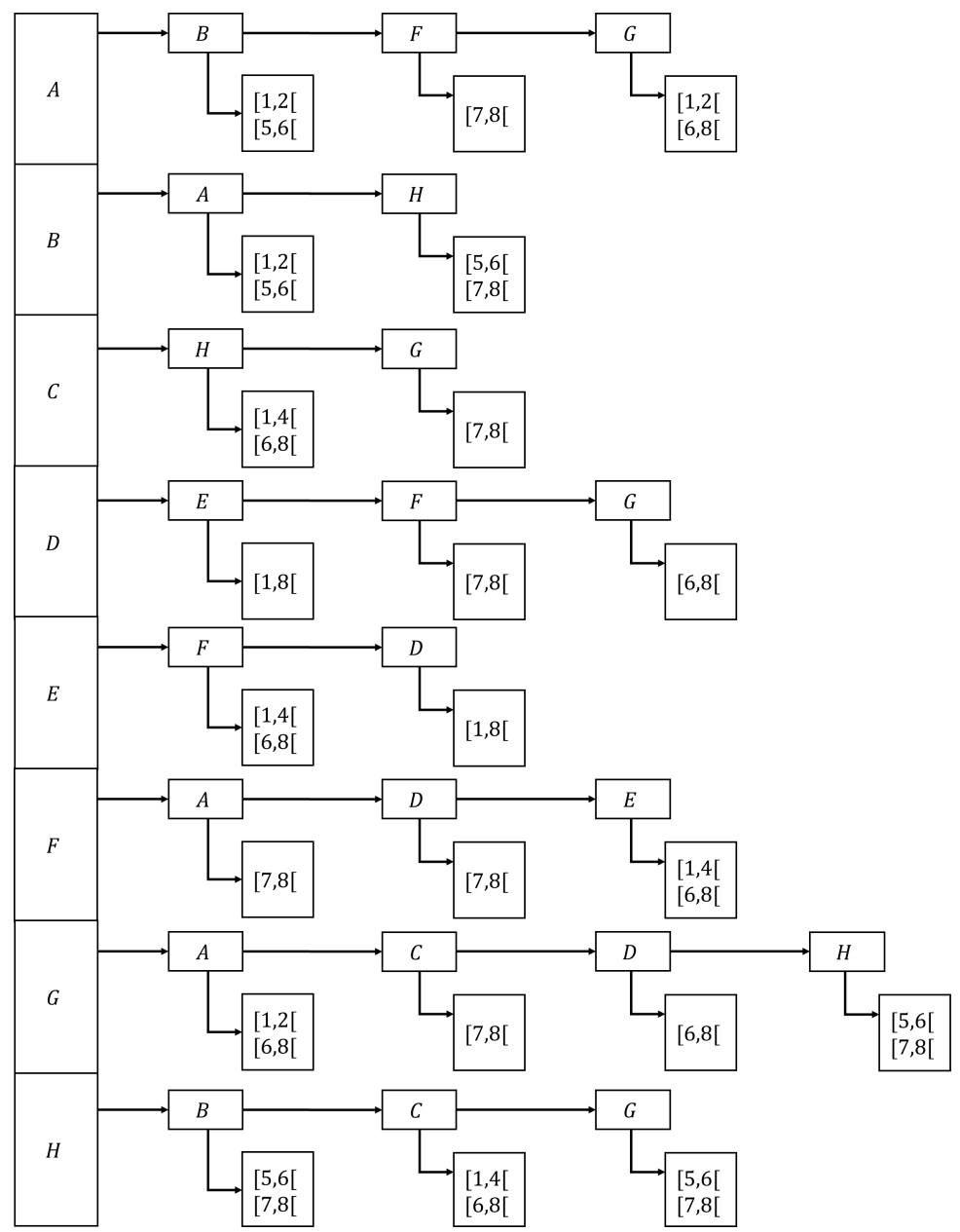

Figure 4: Evolving Graph data structure example.

Second, we assume instantaneous edge traversal. In other words, there is negligible delay or cost for $\zeta$. The assumption of negligible delay is justified for the FSDN that we consider such as bus networks and satellite networks. The two technologies used in vehicular networks are IEEE 802.11p and LTE. The authors of [37] define the mean delay to be the ratio between the summation of all end-to-end delays and the number of received packets. They show that the end-to-end delay with IEEE $802.11 \mathrm{p}$ is less than $100 \mathrm{~ms}$ for $90 \%$ of the beacons with 25 vehicles running on the road network while for 50 vehicles; $40 \%$ of the beacons have a delay less than 100ms. LTE technology is even better, achieving an end-to-end delay less than $60 \mathrm{~ms}$ depending on the beacon 
transmission frequency. Considering that satellite networks have optic links capable of transferring 2,50Gbps over a distance of $2500 \mathrm{~km}$ [10], and cover only about $3 \mathrm{~km}$ in one second, it is reasonable to consider that the duration of connections is longer than end-to-end transmission takes place. A similar calculation holds for buses.

\subsubsection{Connection}

A connection $c=\left(c_{\text {source }}, c_{\text {target }}, c_{\text {start }}, c_{\text {end }}\right)$ corresponds to a temporal edge in an evolving graph $\mathcal{G}$. Basically, a connection expresses the existence of a link between nodes $c_{\text {source }}$ and $c_{\text {target }}$ in the time interval $\left[c_{\text {start }}, c_{\text {end }}[\right.$. There are two fundamental logical restrictions for this definition regarding the endpoints and time. The first one is $c_{\text {source }} \neq c_{\text {target }}$ and the second one is $c_{\text {end }}>c_{\text {start }}$. We say a connection $c$ is active at time $t$ if $c_{\text {start }} \leq t<c_{\text {end }}$.

\subsubsection{Timetable}

A timetable $\mathcal{T}$ is another way to represent an evolving graph: it is a pair $(V, \mathcal{C})$ of a set of nodes $V$ and an ordered sequence of lists of connections $\mathcal{C}=\left\{\mathcal{C}_{0}, \mathcal{C}_{1}, \ldots, \mathcal{C}_{s}\right\}$, where $\mathcal{C}_{i}$ is the set of the connections that start at time $t_{i}$, as Figure 5 shows. We denote the first and last time step in the timetable as $\mathcal{T}_{\text {start }}=t_{0}$ and $\mathcal{T}_{\text {end }}=t_{s}$, respectively.

Figure 6 illustrates an example, and shows the timetable representation of the graph in Figure 3.

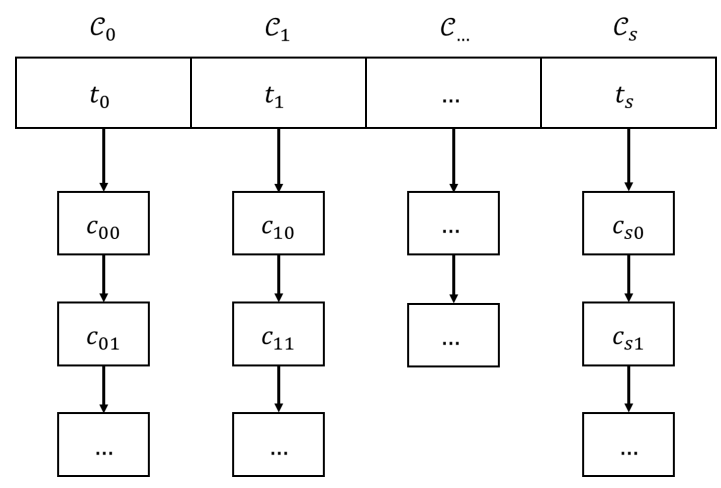

Figure 5: $\mathcal{C}$ data structure. 


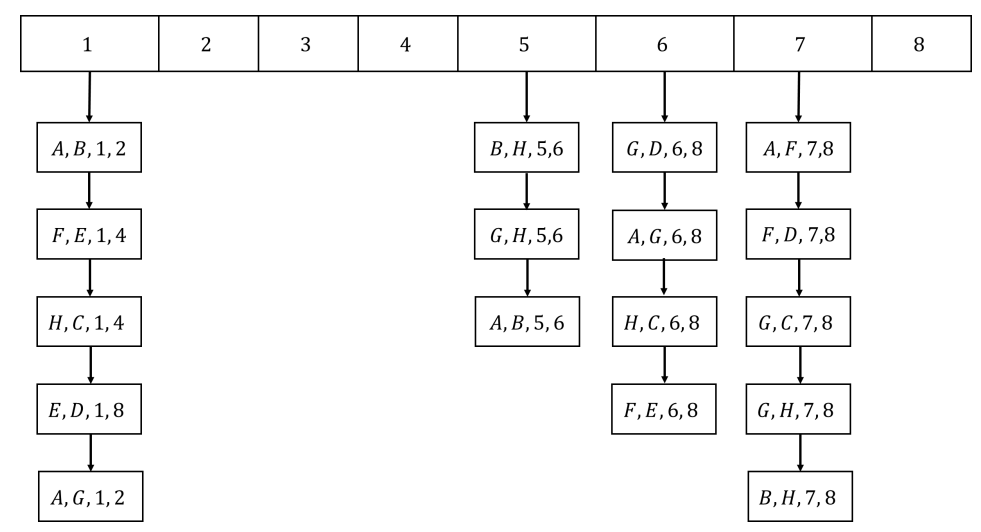

Figure 6: $\mathcal{C}$ data structure example.

\subsubsection{Duplicated timetable}

We define an operation using $\mathcal{T}$ which returns another timetable $\mathcal{T}_{d}$ that we call duplicated timetable. This operation $\mathcal{T}_{d}=\operatorname{duplicate}(\mathcal{T})$ is specified in Algorithm 3. The main particularity of $\mathcal{T}_{d}$ is that each $\mathcal{C}_{t}$ holds the connections that are active at time $t$. It is clear that the size of the duplicated timetable could be much bigger than the original timetable.

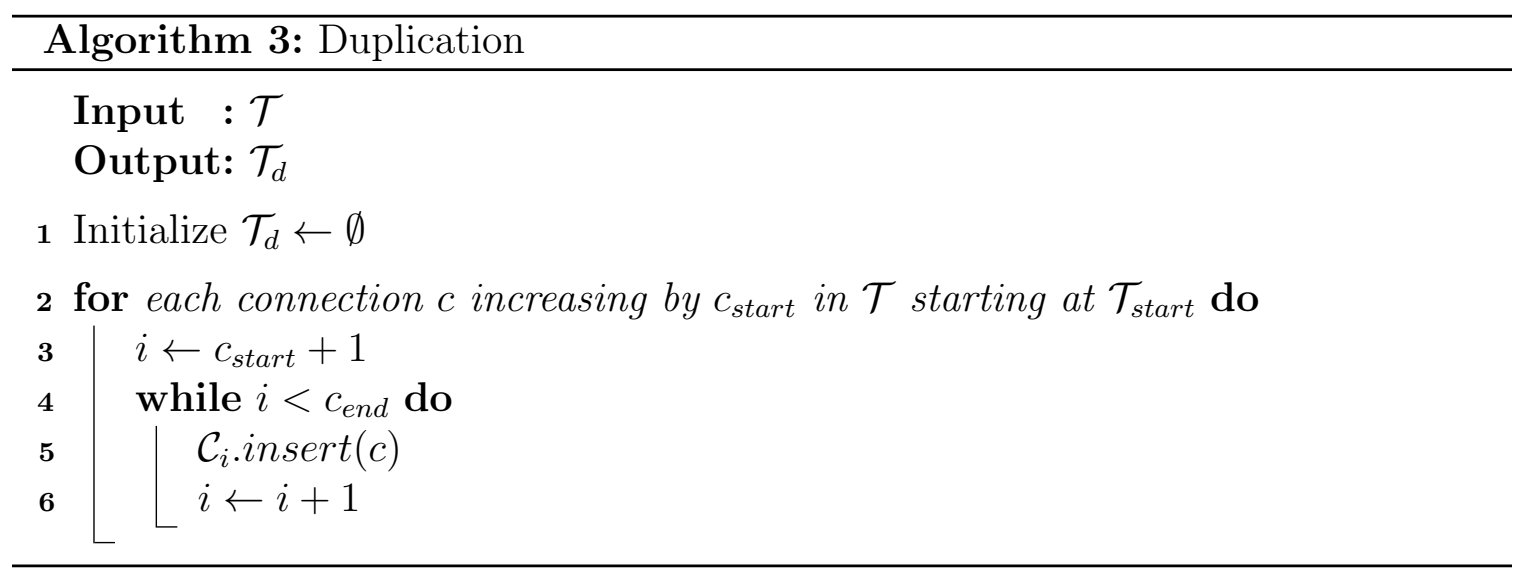

\subsubsection{Journey}

A journey $\mathcal{J}$ (source,target) in an evolving graph $\mathcal{G}$ is a temporal path from source to target, that is, it is a sequence of pairs of nodes $v_{i}$ and time steps $t_{i}$ :

$$
\mathcal{J}(\text { source, target })=\left\{\left(v_{0}, t_{0}\right), \ldots,\left(v_{j}, t_{j}\right)\right\}, v_{i} \in V
$$

where $v_{0}=$ source $\wedge v_{j}=$ target $\wedge t_{i} \leq t_{i+1} \forall 0 \leq i<j$ 
In terms of the timetable representation, note that in order for a packet to travel from node $v_{i}$ to $v_{i+1}$ at time $t_{i+1}$ there must be an active connection $c \in \mathcal{C}$ at time $t_{i+1}$ such that:

$$
c_{\text {source }}=v_{i} \wedge c_{\text {target }}=v_{i+1} \wedge c_{\text {start }} \leq t_{i+1}<c_{\text {end }}
$$

We define $\operatorname{dep}(\mathcal{J})=t_{0}$ and $\operatorname{arr}(\mathcal{J})=t_{j}$. Informally, we call these the departure time, and arrival time of the journey $\mathcal{J}$.

We say a journey $\mathcal{J}$ is foremost if $\operatorname{arr}(\mathcal{J})$ is as small as possible. As shown in [53], there exists a foremost journey such that every prefix of the journey is also a foremost journey; such a journey is called a ubiquitous foremost journey.

We denote by $\delta\left(s, v, t_{q}\right)$, the earliest time that $v$ can be reached departing from $s$ at time $t_{q}$. Then $\delta\left(s, v, t_{q}\right)=t$ if and only if there is a foremost journey $\mathcal{J}=\mathcal{J}(s, v)$ with $\operatorname{dep}(\mathcal{J})=t_{q}$ and $\operatorname{arr}(\mathcal{J})=t$.

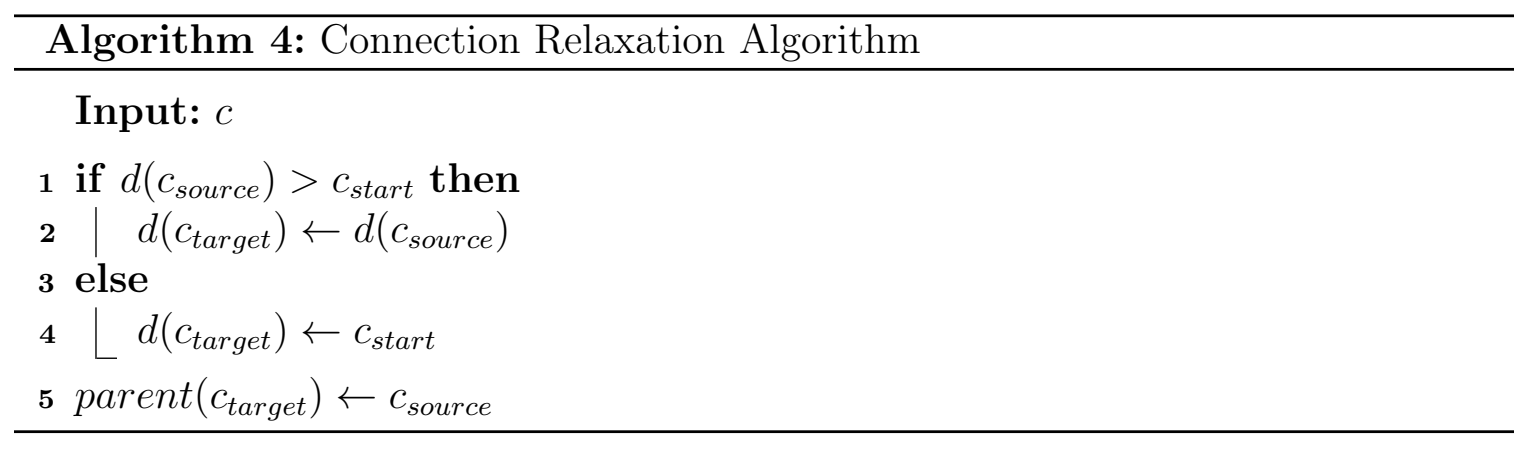

We now describe a fundamental primitive used in our algorithms, called connection relaxation. Recall that the process of edge relaxation in shortest path algorithms such as Dijkstra and Bellman-Ford for static graphs involves checking if going through an edge $(u, v)$ can lower the cost of reaching the vertex $v$, and accordingly updating the estimated cost of reaching $v$. In the evolving graphs that we are investigating, edges have zero weight, but are transient. Therefore, we examine a connection, and if source of the connection has already been reached while the target has not, then we can reset the arrival time at the target appropriately as described in Algorithm 4. The condition $d\left(c_{\text {source }}\right)>c_{\text {start }}$ noted in line 1 , tests if $c_{\text {source }}$ has been reached after the connection $c$ has already started. In this case, $d\left(c_{\text {target }}\right)$ is set to $d\left(c_{\text {source }}\right)$. Otherwise, $c_{\text {source }}$ holds the packet until $c$ starts at which time it can pass it to $c_{\text {target }}$. Thus $d\left(c_{\text {target }}\right)$ is set equal to $c_{\text {start }}$. Afterwards, parent $\left(c_{\text {target }}\right)$ is set to indicate $c_{\text {source }}$ as the predecessor of $c_{\text {target }}$. 
Notice that when Algorithm 4 relaxes a connection, it sets a parent reference from the target of the connection to the source of the connection. While in our description of algorithms, we generally are concerned with the arrival time of the foremost journey, it should be clear that by following the references that each node holds in parent $(v)$, we can extract the corresponding journey from source to target.

\subsection{Ferreira's algorithm with $t_{q}$ support}

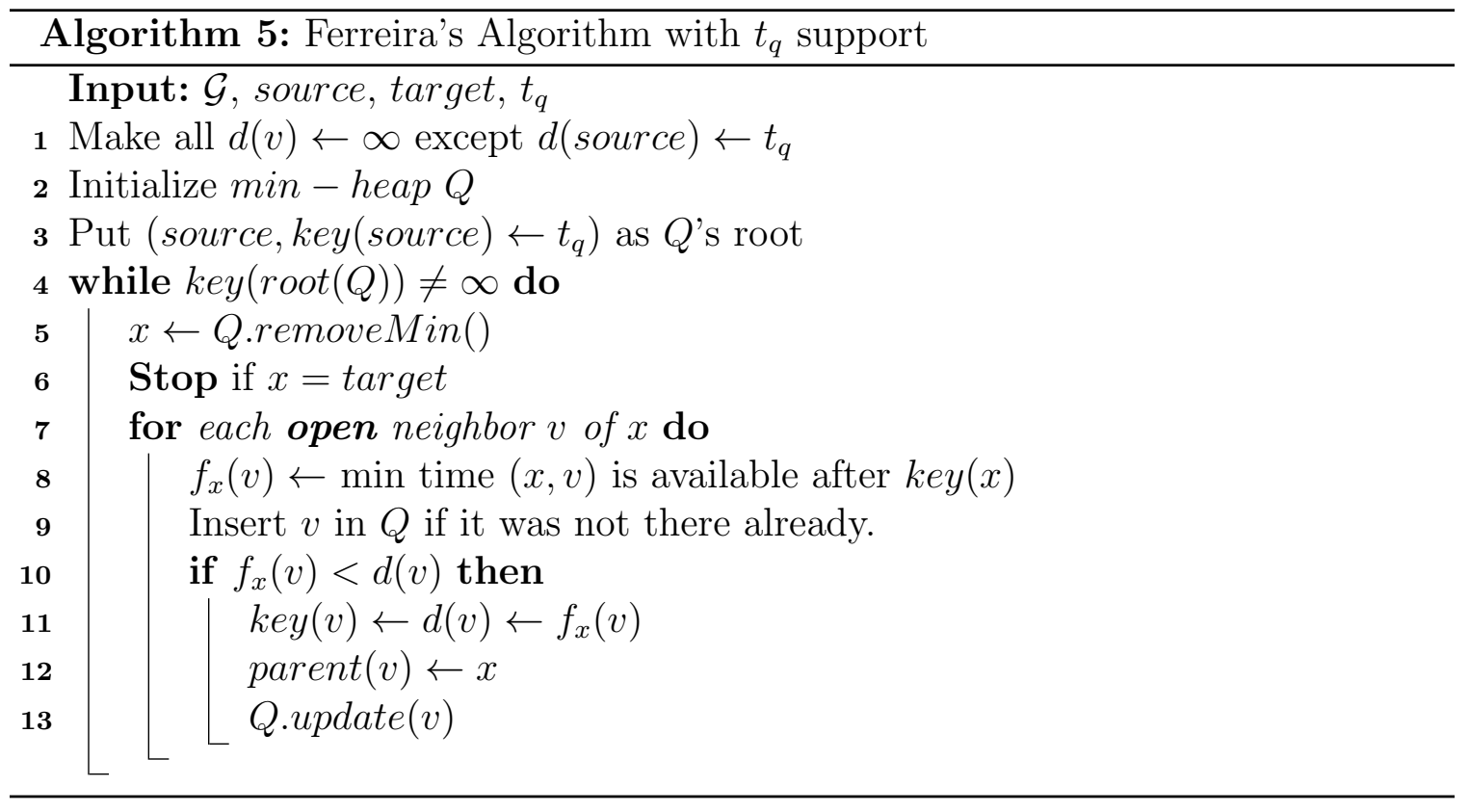

Algorithm 5 shows Ferreira's algorithm [21] that supports $t_{q}$ as input. The difference between Algorithm 1 and Algorithm 5 is that $d$ (source) is set to $t_{q}$ instead of zero. This is because the earliest time that source can be reached is not zero anymore but $t_{q}$. Since, we consider no delay for edge traversing the variables key(source) and $i$ are set to $t_{q}$ instead of one. Also, the no delay assumption is showed in line 13 once a node is relaxed it sets the value of $d(v)$ and $k e y(v)$ equal to $f_{x}(v)$.

Another added feature is the stop statement in line 6. If the node $x$ that is inserted in the shortest path tree is equal to target then it means that the algorithm found the foremost journey $\mathcal{J}$ (source,target). So, it is not necessary to continue running the algorithm.

The complexity of this algorithm which is $\mathcal{O}(|E|(\log I+\log |V|))$ remains the same 
as explained in the previous chapter since its complexity does not depend on $t_{q}$ but in the total number of processed nodes and table look-up for valid schedule times. Also, its correctness proof is the same since instead of starting at time zero, this version starts at time $t_{q}$ which is the earliest time during Algorithm 5 running.

\subsection{Timetable-Based Algorithms}

In this section, we describe a new timetable-based approach to find a foremost journey in an evolving graph. We then describe three new algorithms using this new approach which we call: Pure-Timetable, Hybrid Timetable-Evolving Graph, Timetable with Auxiliary Graph and prove their correctness and tight bounds on their space and time complexities.

The key advantage of our timetable-based approach over Ferreira's algorithm is that we use faster array-based operations on the timetable of connections, rather than priority-queue operations. As in Ferreira's algorithm, we maintain an array $d[]$ containing estimates of the earliest arrival time at every node in the network. The main idea is to process connections in sorted order of the start time of connections. When processing a connection, if one of its endpoints has already been reached, we can relax the connection to obtain the earliest arrival time at the other end of the connection. We will show that as soon as node is reached via a connection for the first time, we will have the earliest arrival time at the node.

The main difficulty that arises is that connections are not eligible to be relaxed when they start, but they could be useful later on. For example, suppose $c_{1}=$ $\left(a, b, t_{1}, t_{1}+2\right)$ and $c_{2}=\left(x, a, t_{1}+1, t_{1}+2\right)$. Then $c_{1}$ appears before $c_{2}$ in the timetable. It is possible that neither $a$ nor $b$ is reachable at time $t_{1}$. However, $x$ may be reachable at time $t_{1}+1$ when connection $c_{2}$ is processed. This implies that $a$ is also reachable at time $t_{1}+1$, and via connection $c_{1}$ which is still active at time $t_{1}+1$, the node $b$ is also reachable at $t_{1}+1$. Thus we cannot simply discard connection $c_{1}$ at time $t_{1}$ if it is not useful then; it may be useful later. It is not hard to see that even with connections starting at the same time, their order in the timetable can make a difference to whether or not they can be immediately useful.

A related difficulty arises from the fact that to find a journey starting at time $t$, we cannot simply start with connections starting at time $t$, as there may be pre-existing 
connections that are still alive at time $t$ that can be used.

Our three algorithms utilize different approaches to solve the two problems described above.

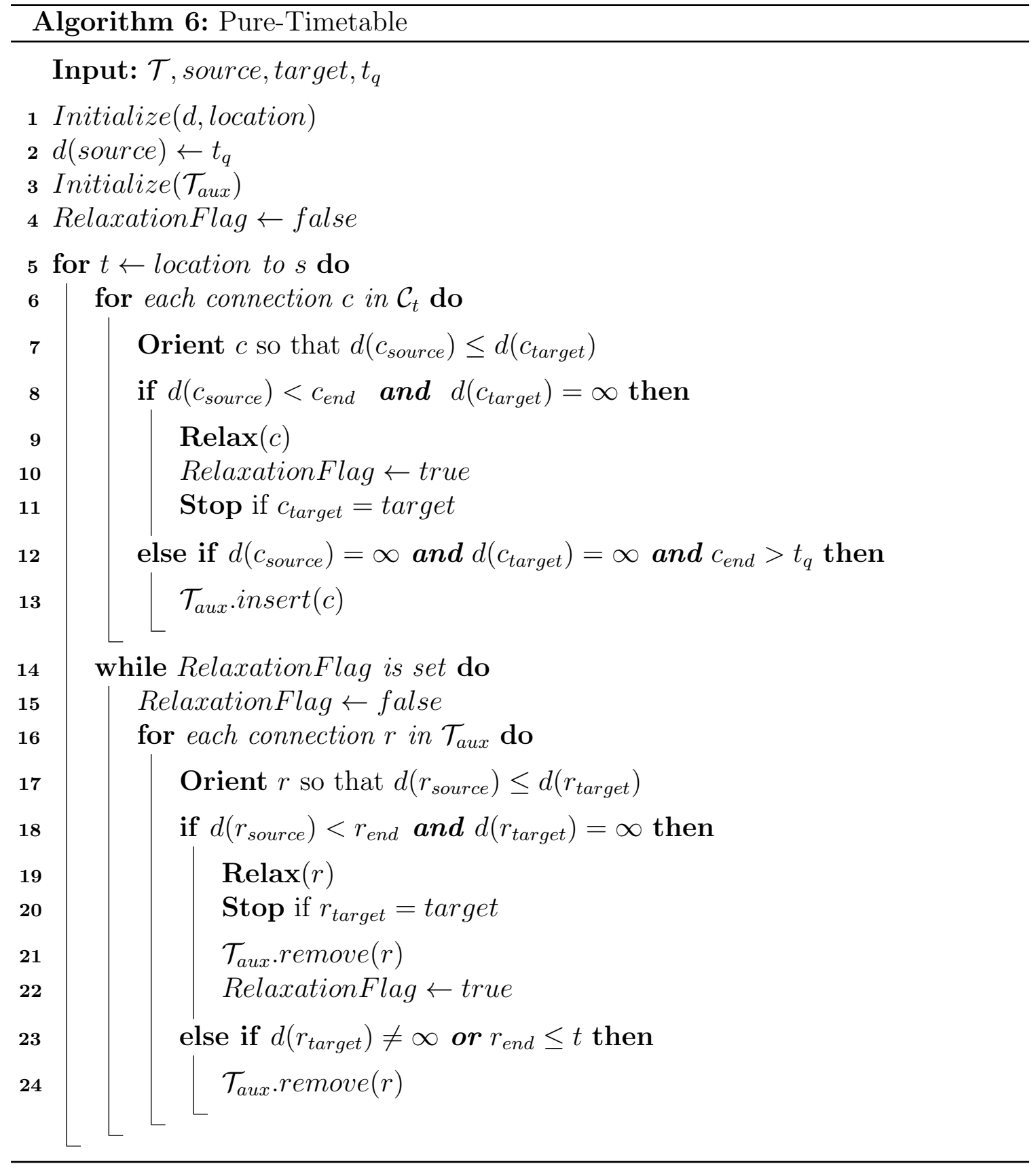




\subsubsection{Pure-Timetable}

The first timetable based algorithm that we describe is Algorithm 6 which we call Pure-Timetable. This approach uses an auxiliary timetable $\mathcal{T}_{\text {aux }}$ to store connections that were ineligible to be relaxed when first processed, but might still be eligible to be relaxed later.

The first step is Initialize(d,location). The initialization of $d$ involves setting $d(v)$ to infinity for every node $v$ element of $V$. The location variable is set to the smallest $t$ such that there is a connection starting at $t$ that is still active at time $t_{q}$. To do this, we use a the duplicated timetable generated with Algorithm 3, in which connections are duplicated in every time step that they are active; this table is used only for this purpose. Next we set $\mathcal{T}_{\text {aux }}$, the auxiliary timetable to empty, and set to false the boolean flag RelaxationFlag, which indicates if there was a relaxation of any edge while processing connections that are active at time $t$. This flag will be used to determine if $\mathcal{T}_{\text {aux }}$ is to be scanned again.

In the event of finding a connection $c$ that can not be relaxed, then there are some conditions to meet in order to save a connection in $\mathcal{T}_{\text {aux }}$. These conditions are $d\left(c_{\text {source }}\right)=\infty, d\left(c_{\text {target }}\right)=\infty$ and $c_{\text {end }}>t_{q}$. In other words, one connection is saved only if both ends; $c_{\text {source }}$ and $c_{\text {target }}$ are not being reached yet and the connection is still active after $t_{q}$. One thing to recall and notice is that connections in $\mathcal{T}$ are ordered by $c_{\text {start }}$ and connections are added to $\mathcal{T}_{\text {aux }}$ as $\mathcal{T}$ is scanned. Then, connections in $\mathcal{T}_{\text {aux }}$ keep the same order that they have in $\mathcal{T}$.

The proof of correctness of our Pure-Timetable algorithm involves the following lemmas that we prove as follows:

Lemma 3.3.1 For every $t$, at the end of the iteration of the outer loop corresponding to time $t$, the table $\mathcal{T}_{\text {aux }}$ contains all connections $r$ satisfying:

$$
d\left(r_{\text {source }}\right)=d\left(r_{\text {target }}\right)=\infty \wedge r_{\text {start }} \leq t \wedge r_{\text {end }} \geq \max \left(t, t_{q}\right)
$$

Proof. The conditions to meet in order to save a connection $r$ in $\mathcal{T}_{\text {aux }}$ are: $d\left(r_{\text {source }}\right)=\infty, d\left(r_{\text {target }}\right)=\infty$ and, since $r \in C_{t}$ we have $r_{\text {end }}>t \geq t_{q}$. In other words, a connection $r$ is saved only if both ends; $r_{\text {source }}$ and $r_{\text {target }}$ have not been reached yet and the connection is still active. Since connections that start after time $t$ have not yet been processed, it is clear that $r_{\text {start }} \leq t$. On the other hand, while 
scanning $\mathcal{T}_{\text {aux }}$, connections that are no longer active are removed. Therefore, at the end of iteration of the outer loop for time $t$, the connections that $\mathcal{T}_{\text {aux }}$ holds are the set of all still-active connections $r$ for which neither endpoint has yet been reached and that are still alive at time $t$.

Lemma 3.3.2 Once $d(v)$ is set to an integer value, it will never change again.

Proof. Note that the $d$-value of any node is set either in line 2 , or inside a call to a Relax procedure; such a procedure is only called in lines 9 and 19 of the algorithm. In both cases, the connection $c$ is relaxed only if its target $v$ satisfies $d(v)=\infty$. Once $d(v)$ is set to an integer value, no connection with $v$ as target can be relaxed again.

Lemma 3.3.3 During the iteration of the outer loop corresponding to time $t \geq t_{q}$, if we call Relax $(c)$, then $d\left(c_{\text {target }}\right)$ is set to $t$.

Proof. First note that when the Relax procedure is called for a connection $c$ in $\mathcal{T}$ (in line 9 ), we can assume inductively that $d\left(c_{\text {source }}\right) \leq t=c_{\text {start }}$, therefore when $c$ is relaxed, $d\left(c_{\text {target }}\right)$ is set to $t$. Now consider the case when the Relax procedure is called for a connection $r$ in $\mathcal{T}_{\text {aux }}$ in line 19. By Lemma 3.3.1, we know that when we started processing connections in $C_{t}$, for all connections in $\mathcal{T}_{\text {aux }}$, we must have had $d\left(r_{\text {source }}\right)=d\left(r_{\text {target }}\right)=\infty$. Since the connection $r$ is now eligible to be relaxed, it must be that some other connection $c$ in $\mathcal{T}$ with $c_{\text {start }}=t$ and $c_{\text {target }}=r_{\text {source }}$ was relaxed. As argued earlier, in this case, $d\left(c_{\text {target }}\right)=d\left(r_{\text {source }}\right)$ must have been set to $t$. Therefore, when $r$ is relaxed, $d\left(r_{\text {target }}\right)$ will be set to $t$.

Lemma 3.3.4 During the iteration of the outer loop corresponding to time $t<t_{q}$, any call to Relax $(c)$ leads to $d\left(c_{\text {target }}\right)=t_{q}$.

Proof. Consider the first iteration of the outer loop corresponding to a time $t<t_{q}$. This means there is a connection $c$ that started before $t_{q}$ but was still alive at time $t$. Since the only node for which the $d$ value is not $\infty$ is source, if neither endpoint of $c$ is source, then the condition for relaxation is not met, and $c$ is added to $\mathcal{T}_{\text {aux }}$. Therefore, the first connection $c$ which will be relaxed must have source as an endpoint, and since $d\left(c_{\text {source }}\right)=t_{q}>t=d\left(c_{\text {start }}\right)$, according to the logic of the relaxation algorithm, 
$d\left(c_{\text {target }}\right)$ is set to $d\left(c_{\text {source }}\right)=t_{q}$. A similar logic applies to subsequent connections that are relaxed in $C_{t}$ for $t<t_{q}$ (even if neither endpoint is source).

Now consider the case when the Relax procedure is called for a connection $r$ in $\mathcal{T}_{\text {aux }}$ in line 19. By Lemma 3.3.1, we know that when we started processing connections in $C_{t}$, for all connections in $\mathcal{T}_{\text {aux }}$, we must have had $d\left(r_{\text {source }}\right)=d\left(r_{\text {target }}\right)=\infty$. Since the connection $r$ is now eligible to be relaxed, it must be that some other connection $c$ in $\mathcal{T}$ with $c_{\text {start }}=t$ and $c_{\text {target }}=r_{\text {source }}$ was relaxed. As argued earlier, in this case, $d\left(c_{\text {target }}\right) \leftarrow d\left(r_{\text {source }}\right)$ must have been set to $t_{q}>t$. Therefore, when $r$ is relaxed, $d\left(r_{\text {target }}\right)$ will be set to $t_{q}$.

We now show that Algorithm 6 correctly sets $d(v)=\delta\left(s, v, t_{q}\right)$, recall that $\delta\left(s, v, t_{q}\right)$ is the earliest time that $v$ can be reached departing from $s$ at time $t_{q}$, for every vertex $v$ in the evolving graph.

To do this, we need some new notation. We define $T\left(s, t_{q}, t\right)$ to be the set of all nodes whose earliest arrival time is $t$, starting from $s$ at time $t_{q}$. That is,

$$
T\left(s, t_{q}, t\right)=\left\{u \mid t=\delta\left(s, u, t_{q}\right)\right\}
$$

Lemma 3.3.5 For every $t$ with $t_{q} \leq t \leq t_{s}$, at the end of the iteration corresponding to time $t$ in Algorithm 6, for every $u \in T\left(s, t_{q}, t\right)$, we have $d(u)=\delta\left(s, u, t_{q}\right)=t$.

Proof. We give a proof by contradiction. Let $t$ be the minimum start time such that there exists a $v \in T\left(s, t_{q}, t\right)$ such that after the iteration of the outer loop corresponding to time $t$, we have $d(v) \neq t$. We claim that then $d(v)=\infty$. First note that the value of $d(v)$ can only be set according to connections being relaxed along a journey from $s$ to $v$, and therefore, it is impossible that $d(v)<t$. By Lemma 3.3.2, it cannot be that $d(v)$ was set to an integer value $>t$. We conclude that $d(v)=\infty$.

If there are many such vertices, we pick $v$ so that it has a foremost journey $\mathcal{J}$ starting from $s$ at time $t_{q}$ such that $\mathcal{J}$ has the minimum number of hops among all such vertices. Note that there may be many such min-hop foremost journeys, we pick an arbitrary one of them. Now consider the previous vertex $u$ on this journey. It follows from the definition of $v$ as a node with a min-hop foremost journey with arrival time $t$ that $d(u)$ must be set correctly to $\delta\left(s, u, t_{q}\right)$. Note that there must be 
such a previous vertex, as $v \neq$ source, since $d$ (source) is correctly set to $t_{q}$ by the algorithm. We consider the following two cases for $u$.

1. $\delta\left(s, u, t_{q}\right)=t$ : Then there must be a connection $c$ from $u$ to $v$ that is active at time $t$, in other words, $c_{\text {end }}>t$.

If $c_{\text {start }}=t$, then $c$ would be processed while scanning connections in $\mathcal{T}$. If $d(u)=t$ at the time $c$ is processed, then the connection $c$ would be relaxed and $d(v)$ would be set to $t$, a contradiction. If $d(u) \neq t$ at the time $c$ is processed, then it must be that $d(u)=\infty$. Therefore $c$ would be added to $\mathcal{T}_{\text {aux }}$. By assumption $d(u)$ is set to $t$ during some iteration of the while-loop while $\mathcal{T}_{\text {aux }}$ is processed. In the next iteration of the while loop, the connection $c$ would be relaxed and $d(v)$ would be set to $t$, a contradiction.

If instead $c_{\text {start }}<t$ then since $d(u)=d(v)=\infty$ and $c_{\text {end }} \geq t$, when the outer loop iteration corresponding to time $t$ starts, $c$ would be part of $\mathcal{T}_{\text {aux }}$ by Lemma 3.3.1. As in the previous case, by assumption $d(u)$ is set to $t$ during some iteration of the while loop in which $\mathcal{T}_{\text {aux }}$ is processed. In the next iteration of the loop, the connection $c$ is relaxed and $d(v)$ would be set to $t$.

2. $\delta\left(s, u, t_{q}\right)=t^{\prime}<t$ : By the minimality of $t$, we infer that the value of $d(u)$ is set to $t^{\prime}$. Also, there is an active connection $c$ from $u$ to $v$ at time $t$. This connection must start at time $t$, otherwise $v$ would be reachable before time $t$. Therefore $c$ would be found and relaxed during $\mathcal{C}_{t}$ scanning since $d(u)$ is already set to $t^{\prime}$ which is less than $t$. According to the relaxation logic shown in Algorithm 4 $d(v)$ is set to $t$, a contradiction.

Observing that the size of $\mathcal{T}_{\text {aux }}$ never exceeds that of $\mathcal{T}$, we are ready to prove the main theorem of this section:

Theorem 3.3.1 Algorithm 6 finds the foremost journey from any source node to any target node in an evolving graph $G=(V, E)$ with timetable $\mathcal{T}$ in time $\mathcal{O}(|V||E|+|\mathcal{T}|)$, using space $\mathcal{O}(|\mathcal{T}|)$. 
Proof. The correctness of Algorithm 6 follows from Lemmas 3.3.1 to 3.3.5. We now prove an upper bound on the time complexity. First we note that $\mathcal{T}_{\text {aux }}$ can be implemented as a doubly linked list so that insertion and deletion operations can be performed in $\mathcal{O}(1)$ time. Also, we can ensure that its size is always at most $|E|$, by checking for every edge, before inserting a connection corresponding to the edge, if such a connection already exists, and if it does, simply updating with the new connection. It is clear that every instruction in the inner for loop can be implemented in $\mathcal{O}(1)$ time, for a total cost of $\mathcal{O}(|\mathcal{T}|)$.

It remains to analyze the complexity of the while loop. Notice that the while loop is executed only when an edge is relaxed, that is, when the $d$ value of a node is changed. By Lemma 3.3.2, once the $d(v)$ value is set it will never be set again. So the condition for the while loop to execute can be satisfied at most $|V|-1$ times. Together with the fact that the size of $\mathcal{T}_{\text {aux }}$ is at most $|E|$, and each instruction in the for-loop scanning $\mathcal{T}_{\text {aux }}$ can be executed in $\mathcal{O}(1)$ time, we conclude that the total time taken to execute the while loop over all iterations of the outer loop is $\mathcal{O}(|V||E|)=\mathcal{O}\left(|V|^{3}\right)$. We have shown that the total time taken by Algorithm 6 is $\mathcal{O}(|V||E|+|\mathcal{T}|)$.

We now give in Figure 7 an example of an evolving graph on which Algorithm 6 takes $\Omega(|V||E|+|\mathcal{T}|)$ demonstrating that the above analysis is tight. The base graph consists of a path of $n / 2$ nodes connected to a clique of $n / 2$ nodes (i.e. $K_{n / 2}$ ). The schedule of edges is described as follows: the edges in the clique are always alive, while on the path, the connection between node $i$ and node $i+1$ starts only in time step $i$. In this example we start at time step zero, so at the end of processing connections starting at time step 0 , the size of $\mathcal{T}_{\text {aux }}$ is equal to number of edges in $K_{n / 2}$, that is, there are $\Theta\left(n^{2}\right)$ connections in $\mathcal{T}_{\text {aux }}$. In each of the subsequent $n / 2$ time steps, exactly one connection is processed and relaxed, which necessitates scanning the entire $\mathcal{T}_{\text {aux }}$. However, none of these iterations causes any connection to be removed from $\mathcal{T}_{\text {aux }}$, which therefore has size $n / 2(n / 2-1)$ for $n / 2$ steps. The total cost paid is lower bounded by $n / 2(n / 2)(n / 2-1)=\Theta\left(n^{3}\right)=\Theta(|V||E|)$.

In this context the total cost of the previous example is $\Theta\left(V^{3}\right)$.

\subsubsection{Hybrid Timetable-Evolving Graph}

In this section, we describe a hybrid algorithm that uses both a timetable and the evolving graph representation of a dynamic network. The pseudocode is given in 


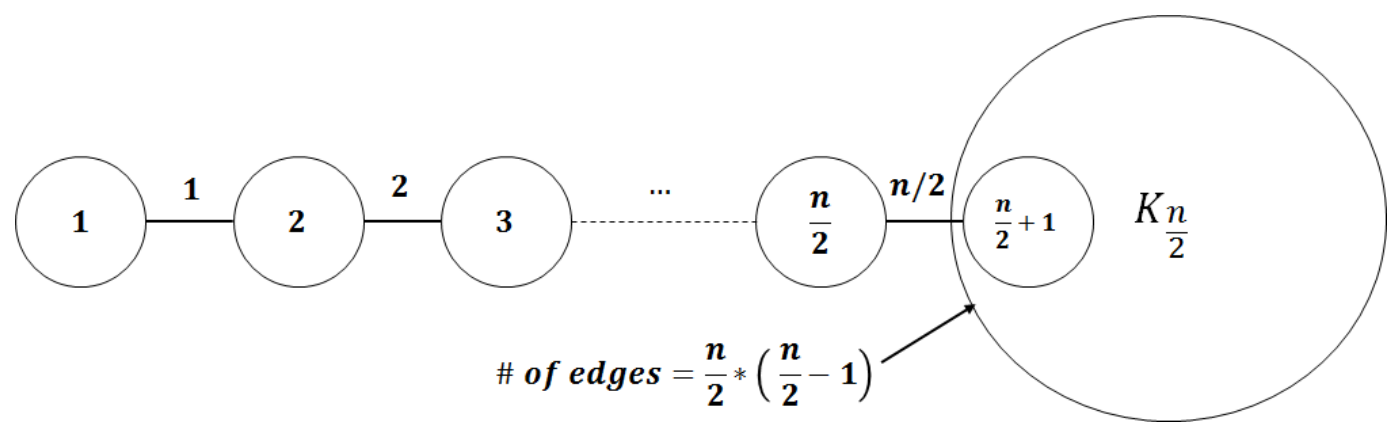

Figure 7: Complexity analysis example.

Algorithm 7. Like Algorithm 6, we process connections in order of start time from a timetable. Instead of storing potentially useful connections in an auxiliary timetable, in this new algorithm, while we process all connections starting at time $t$, we store in a queue any vertices whose $d$-values are changed. Subsequently, we explore the connected components containing these vertices in the graph created by connections that are alive at time $t$, by doing a breadth-first search starting at these nodes. To find the connections that are alive at time $t$, we use the evolving graph data structure of Ferreira [53]. Note that unlike in Pure-Timetable, here we start scanning the timetable at the first time there is a connection starting after time $t_{q}$.

We proceed to prove the correctness of the algorithm.

Lemma 3.3.6 Algorithm 7 sets $d(u)=\delta\left(s, u, t_{q}\right)=t_{q}$ for every node $u \in T\left(s, t_{q}, t_{q}\right)$ after the execution of line 6 .

Proof. The only node in $Q$ when ExploreConnectedComponent is called in line 6 is source where $d($ source $)=t_{q}$. It is easy to see that ExploreConnectedComponent simply performs a BFS-like procedure, only considering connections that are alive at time $t_{q}$. Thus, every node $u$ for which there is a journey from source at time $t_{q}$ is explored, and $d(u)$ is set to $t$.

Lemma 3.3.7 For every $t>t_{q}$, at the end of the iteration of the main loop corresponding to time $t$, Algorithm 7 sets $d(u)=\delta\left(s, u, t_{q}\right)=t$ for every node $u \in$ $T\left(s, t_{q}, t\right)$.

Proof. We give a proof by induction. Assume inductively that when we start the iteration of the loop for some $t>t_{q}$, we have $d(u)=\delta\left(s, u, t_{q}\right)=t^{\prime}$ for every node 


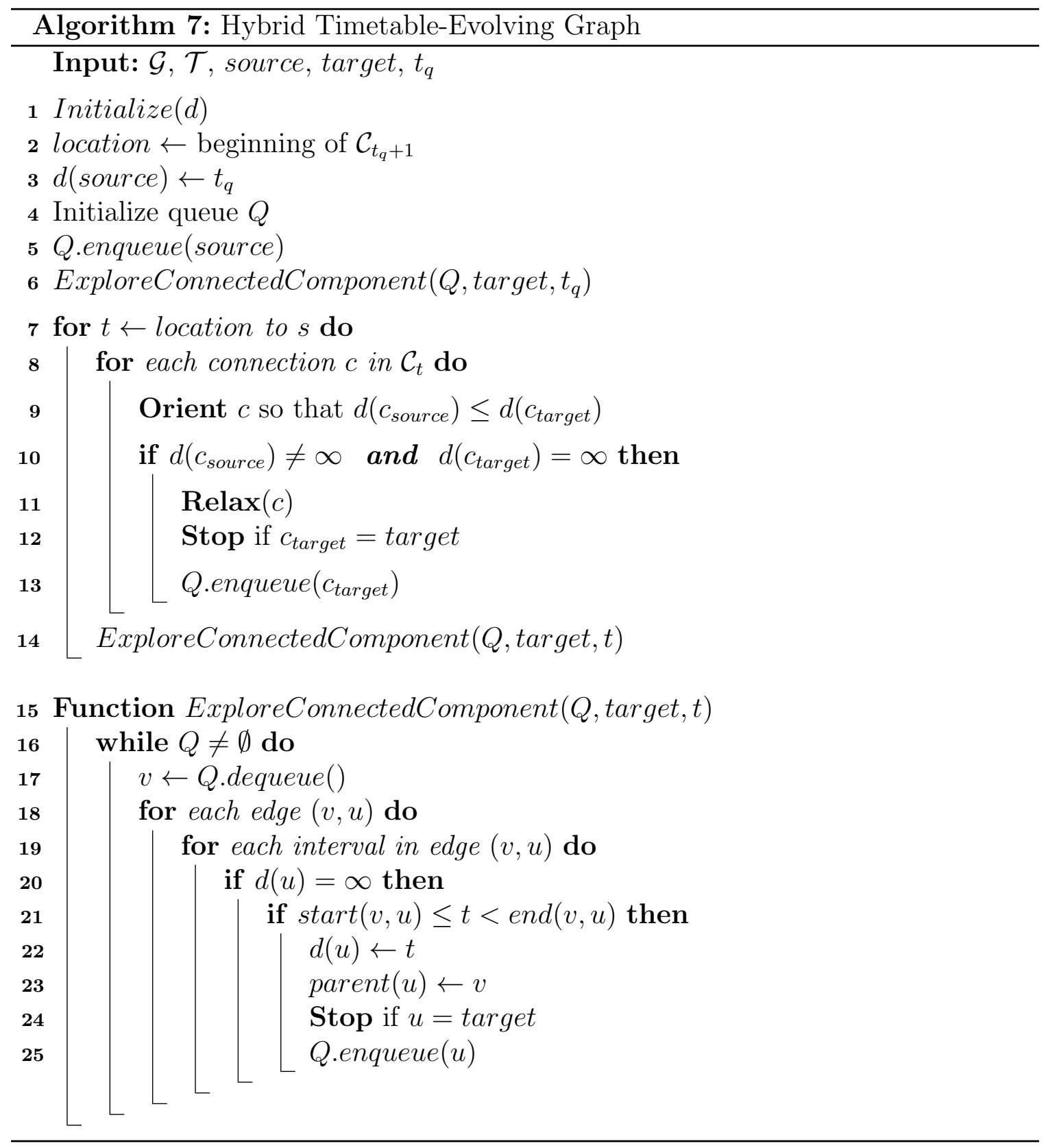

$u \in T\left(s, t_{q}, t^{\prime}\right)$ for every $t^{\prime}<t$. Note that the base case follows from Lemma 3.3.6. Observe that $Q$ is empty at this point, since the termination condition of the while loop in ExploreConnectedComponent is that $Q$ is empty. Consider a vertex $v \in$ $T\left(s, t_{q}, t\right)$ which has a predecessor $u \in T\left(s, t_{q}, t^{\prime}\right)$ with $t^{\prime}<t$ in a foremost journey from $s$. Then there must be a connection $c$ from $u$ to $v$ starting at time $t$. By the inductive assumption, we have $d(u)=t^{\prime}$, and after relaxing the connection $c$, $d(v)=t$, and $v$ will be inserted into the queue. It is clear that any edge relaxed while 
scanning connections in $C_{t}$ results in $d(v)$ being set to $t$ for some vertex $v$. Therefore all vertices in $Q$ have $d$-value equal to $t$ after all connections in $C_{t}$ are scanned. When ExploreConnectedComponent is called, once again, a BFS is performed using connections that are alive at time $t$ and any vertex $u$ that is reachable from a vertex $v$ in $Q$ will have $d(u)=t$. This finishes the proof.

Theorem 3.3.2 Algorithm 7 finds the foremost journey from any source node to any target node in an evolving graph $G=(V, E)$ with timetable $\mathcal{T}$ in time $\mathcal{O}(|E| \log I+$ $|V|+|\mathcal{T}|)$ using space $\mathcal{O}(|\mathcal{T}|)$.

Proof. The correctness of the algorithm follows from Lemmas 3.3.6 to 3.3.7. We proceed to analyze Algorithm 7 in terms of complexity. The main challenge is to analyze the total time taken by ExploreConnectedComponent; it is easy to see that all other operations take at most $\mathcal{O}(|\mathcal{T}|+|V|)$ time. Observe that nodes are enqueued only one time because lines $5,13,25$ enqueue node $v$ only if $d(v)$ is set. Thus the total time dedicated to queue operations over the entire algorithm is equal to $\mathcal{O}(|V|)$. For each dequeued node, we explore the schedule of each neighbor using binary search. So, in total there are $|E|$ edges and the maximum number of time intervals that an edge is active is $I$. Thus, the algorithm spends in the worst case $\mathcal{O}(|E| \log I+|V|+|\mathcal{T}|))$. Finally, observe that the evolving data structure uses constant space for every entry in the timetable, and has size $\mathcal{O}(|\mathcal{T}|)$.

The previous analysis is tight: take an evolving graph whose base graph is a path, which has only one edge active per time step. Then there is one connection that is found in each iteration of the main loop, and only one node whose $d$-value is set, and which is added to the queue. Afterwards, that node is dequeued and its neighbors have to be explored, which takes $\Omega(|E| \log I)$ time. Clearly we also need $\mathcal{O}(|V|+|\mathcal{T}|)$ time to queue the vertices and scan the timetable.

\subsubsection{Timetable with Auxiliary Graph}

Our last algorithm, called Timetable with Auxiliary Graph, combines the merits of the previous algorithms. Instead of storing connections that may be useful later in a separate table as in Pure-Timetable, we create and maintain a graph of currently active connections, and explore this graph, which is of size $|E|$, rather than exploring 


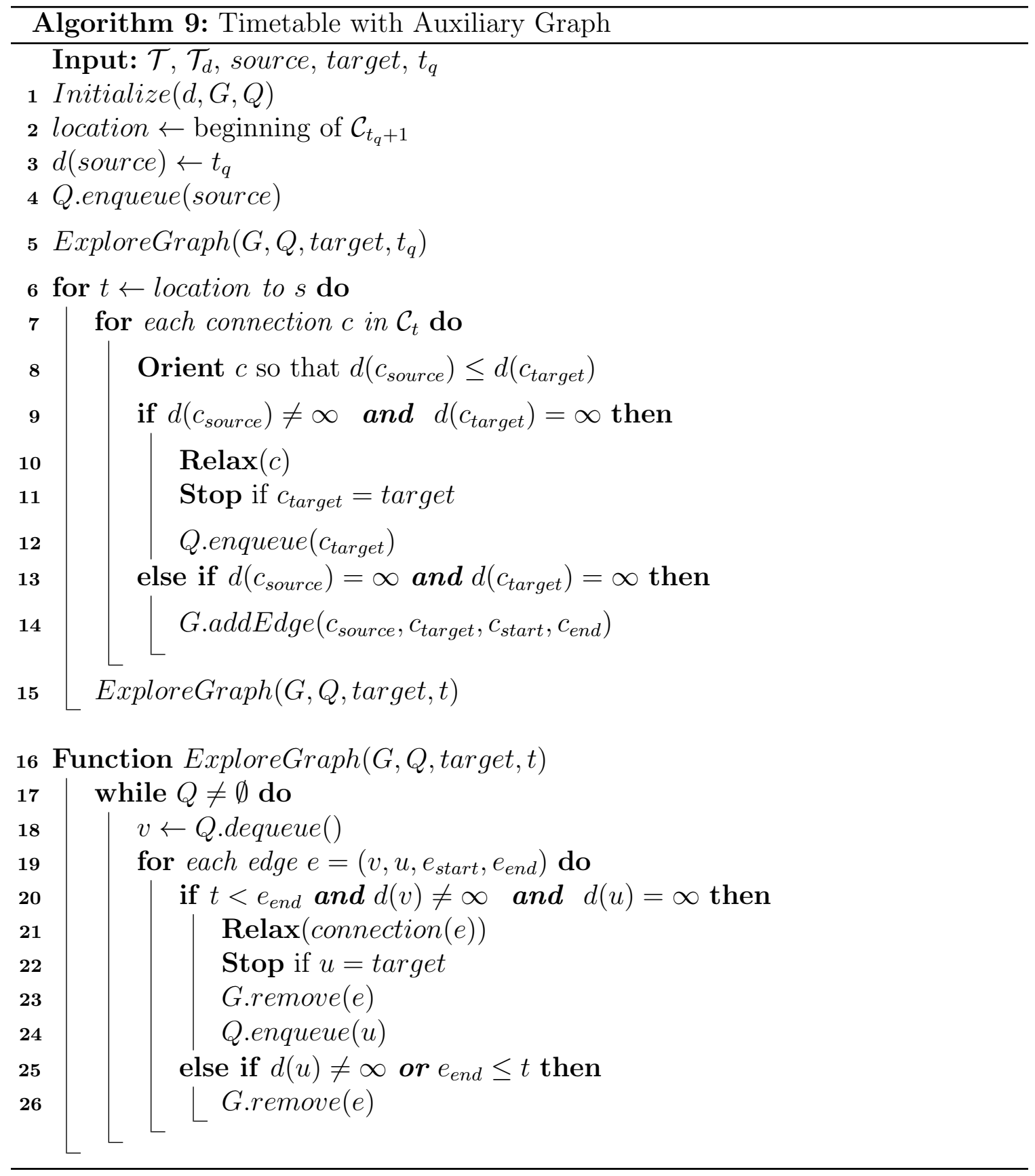

the entire evolving graph as in Hybrid Timetable-Evolving Graph. The pseudocode is given in Algorithm 9. The correctness can be proved in a very similar way to the previous algorithms, and the upper bound on the running time follows from the fact that exploration occurs in a graph of size $|E|$; the complexity analysis is otherwise identical to Algorithm 7. The auxiliary graph uses space $\mathcal{O}(\min (|E|,|\mathcal{T}|))$. We obtain: 
Theorem 3.3.3 Algorithm 9 finds the foremost journey from any source node to any target node in an evolving graph $G=(V, E)$ with timetable $\mathcal{T}$ in time $\mathcal{O}(|E|+|V|+$ $|\mathcal{T}|)$, using space $\mathcal{O}(|\mathcal{T}|)$.

\subsection{Journey extraction}

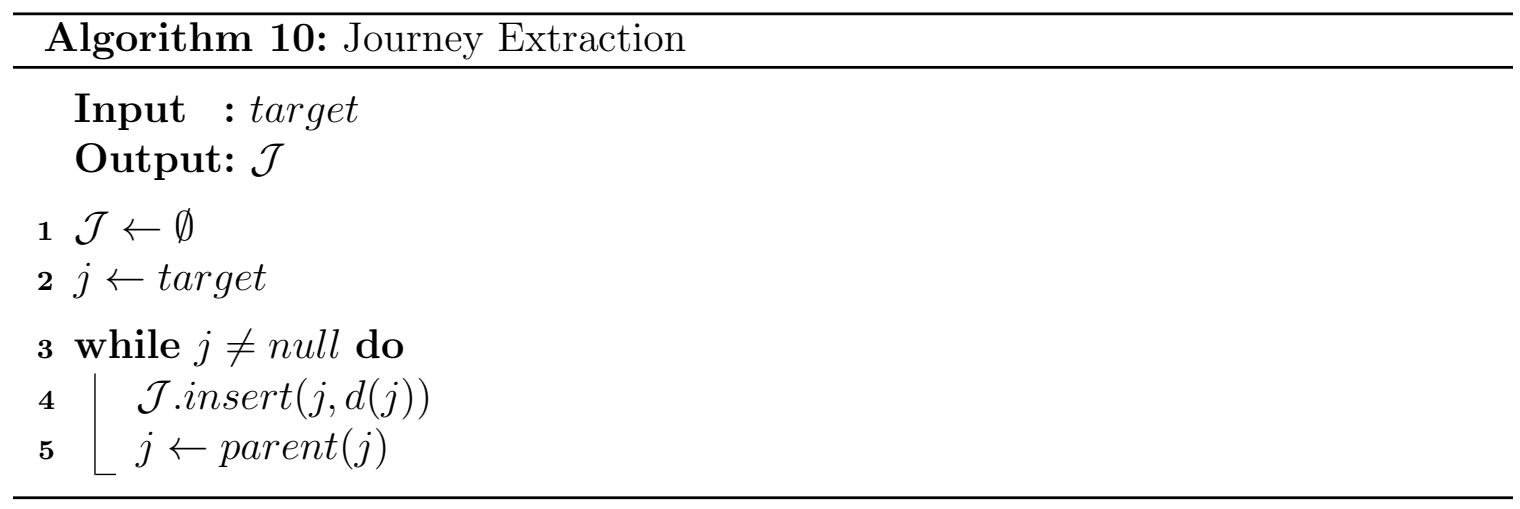

Algorithm 10 shows how we extract a journey after any presented algorithm in this work finds the target node. Each node has a reference to its predecessor which is set during the journey calculation. The reference is saved as parent, so it is enough to follow those references until there is an null value, as line 3 tests. The output is a linked list $\mathcal{J}$ that describes how to reach the target node starting from the source node. 


\section{Chapter 4}

\section{Data sets}

In this chapter we describe the procedure that we follow to generate evolving graphs and timetables, the data sets we use, and their characteristics. In Section 4.1, we present three different data sets which provide information about the position of nodes, and describe how we generate evolving graphs from them. In Section 4.2 once the data structures are generated for each data set; we describe some of their characteristics such as time slots, number of connections, presence ratio, dynamic ratio.

\subsection{Evolving graph and timetable generation}

We use three different data sets in our experiments. The first one is SUMO; it stands for Simulation of Urban Mobility [1], and refers to a well-known simulator that simulates the mobility of vehicles in a given road network. The second one is STK which stands for Systems Tool Kit [11]. This provides information about the position of real satellites all over the world. The last one is Société de Transport de Montréal (STM) [14]. It gives the real-time position of buses in the public transportation system of Montréal, Canada. Thus, we have three different type of nodes: vehicles, satellites and buses. Given a set of nodes and their positions, and a transmission range, we calculate edges and connections based on the euclidean distance between each pair of nodes. If this distance is within the transmission range at a given time, then there is a connection between the pair of nodes. The nodes are said to have an edge between them if there is ever a connection between them. 
We decided to include these three data sources for the following reasons: mobility nature, type and number of nodes. Regarding the mobility nature, SUMO provides random mobility while STK and STM have real mobility. We consider different numbers of buses in SUMO and STK networks, but the number of buses in STM is fixed; we always use the entire set of buses in our experiments.

We now give more details on each of these data sets.

\subsubsection{SUMO}

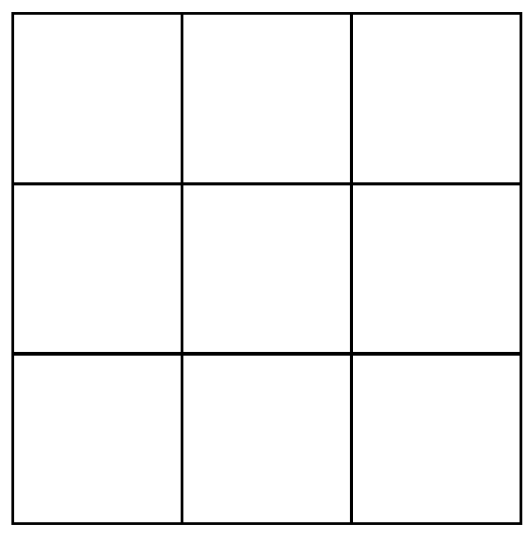

Figure 8: Road network for SUMO simulation.

SUMO is an open source vehicle traffic simulator that includes tools such as netgenerate, jtrrouter, randomTrips.py to model and simulate traffic [1].

Figure 9 shows the schema to obtain the position of each vehicle and parse it into a Evolving Graph and Timetable. The tool netgenerate outputs road networks represented as graphs where intersections are nodes and streets are edges. We use netgenerate to have the road network shown in Figure 8 saved as a xml file called net.net.xml. This road network is a grid, each cell is a square of $2000 \mathrm{~m}$ length.

A trip is a pair of source and destination edges in the road network. We use randomTrips.py which is part of SUMO simulation suite to generate random trips for each vehicle. The trips are saved in file called flows.xml. A route in SUMO is a description of the edges that a vehicle has to take to depart from a given source edge and arrive to a given destination. To generate the route for each vehicle we use jtrrouter which is a SUMO tool to calculate the routes for each vehicle. The file jtrrouter.jtrrcfg provides configuration parameters like turning ratios, loop allowance and all destinations acceptance. We provide 25/50/25 as turning ratio. It 
means a vehicle turns right with probability $25 \%$, goes straight with probability $50 \%$ and turns left with probability 25\%. Also, we allow loops in route calculation. Finally, we have routes. $x \mathrm{ml}$ which encodes the edges that each vehicle has to take during its trip.

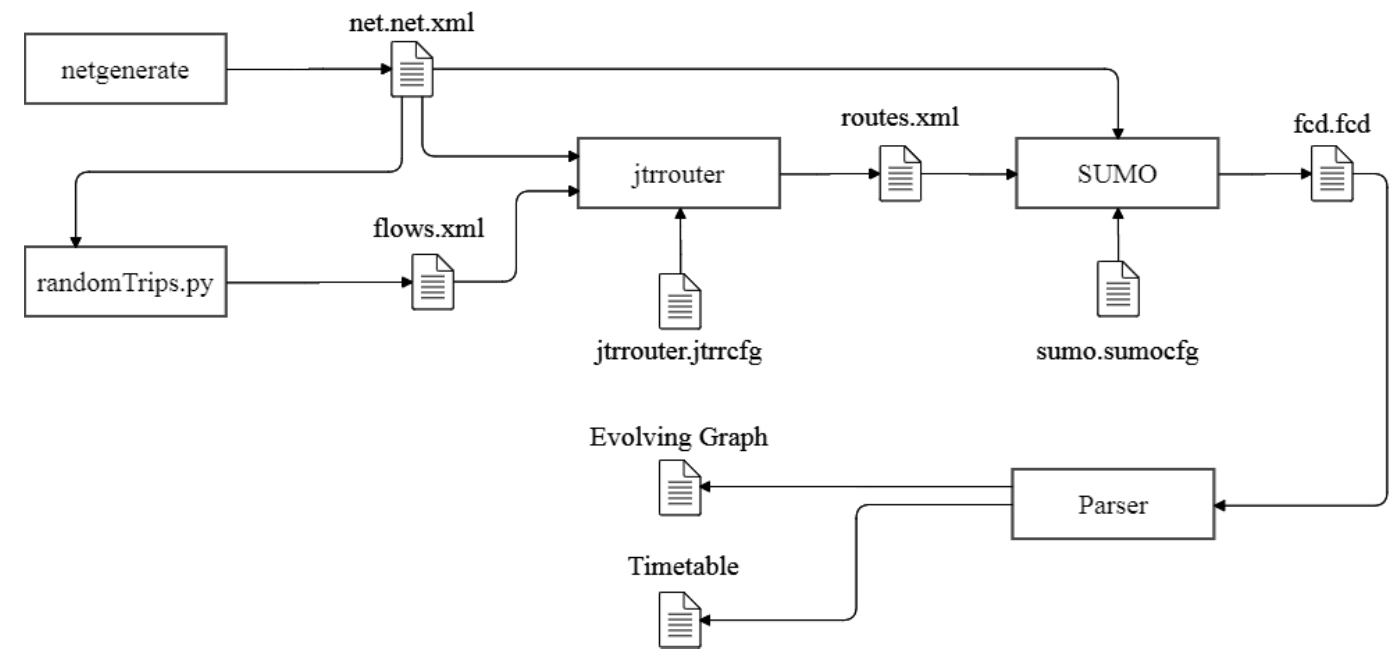

Figure 9: SUMO data parsing.

The way we provide the input to SUMO is shown in Figure 9. The sumo.sumocfg file is a configuration file where we specify the simulation time. It starts at time 0 and ends at time 120, each time step represents one second in SUMO. The output is $f c d . f c d$ which has the position of each vehicle at every time step. So, our Parser module calculates the edges between each vehicle given a transmission range at every time step and outputs a Evolving Graph and Timetable files.

We have 10 different densities of nodes and 14 transmission ranges. The first set of nodes has 100 nodes and the last one has 1000 nodes, in between there are set of nodes in 100 steps. The transmission range starts with $200 \mathrm{~m}$ up to $1500 \mathrm{~m}$ in $100 \mathrm{~m}$ steps. Each density of nodes has 100 different random mobility SUMO simulations running for 120 seconds on the road network shown in Figure 8 whose side is $6000 \mathrm{~m}$ long. For instance, Figure 10 shows different density of nodes with different transmission ranges at time step 60 .

As a result, if we combine every density of nodes with every transmission range then we have 14000 different Evolving Graphs and Timetables to run our experiments. Each experiment chooses 100 random pairs of source - target nodes and a 
random $t_{q}$. Therefore, overall we have 1400000 time measurements for each algorithm. An important point to note is that a source-target pair is only included in the calculation of averages if the target is reachable from the source node; if not, it is simply discarded, and another pair is chosen. To determine reachability, we simply run Ferriera's algorithm.

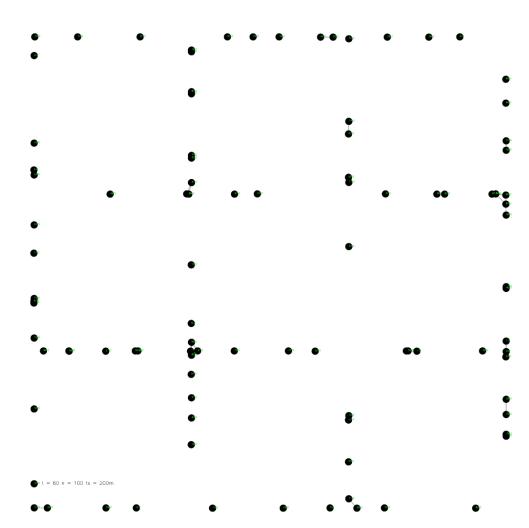

(a) 100 nodes $-200 \mathrm{~m}$.

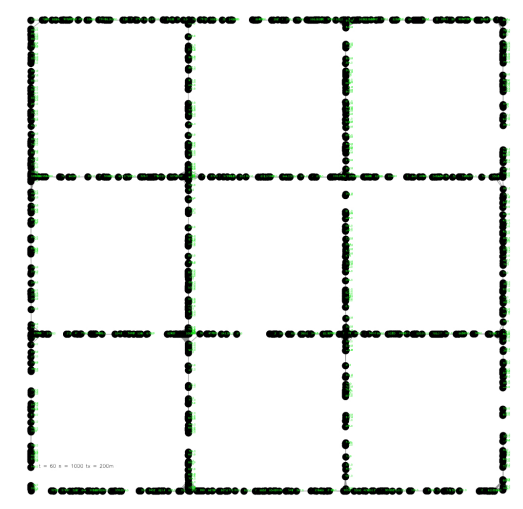

(c) 1000 nodes $-200 \mathrm{~m}$.

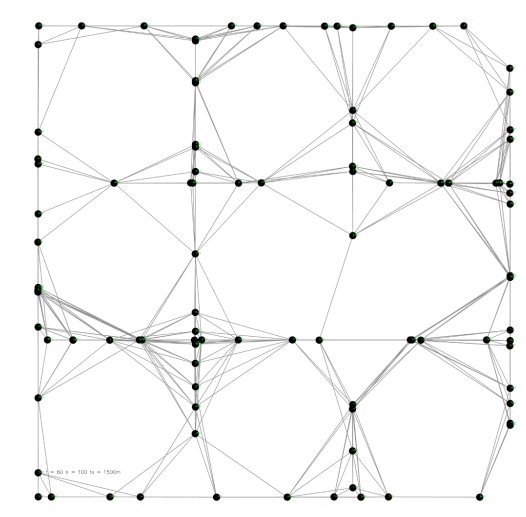

(b) 100 nodes $-1500 \mathrm{~m}$.

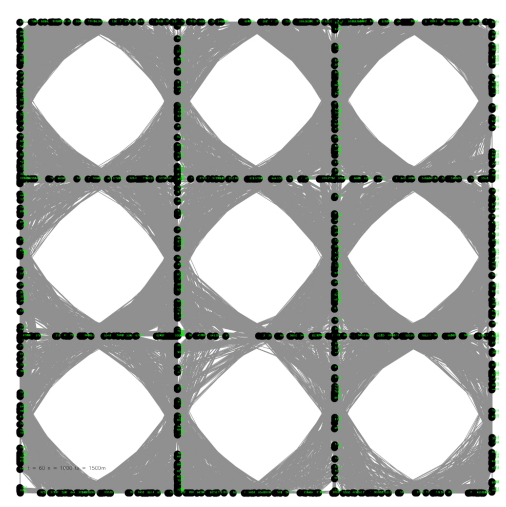

(d) 1000 nodes $-1500 \mathrm{~m}$.

Figure 10: SUMO different density of nodes and transmission ranges.

\subsubsection{STK}

The evolving graph and timetable generation for the STK data source is simple. Figure 11 shows that STK outputs a file called positions.csv. It has the position of each satellite at every time step as a three-dimensional coordinate. Then, to generate the evolving graph and timetable we calculate the euclidean distance at every time step between every pair of satellites. If the distance is within a given transmission range then we consider the existence of a connection between them. 


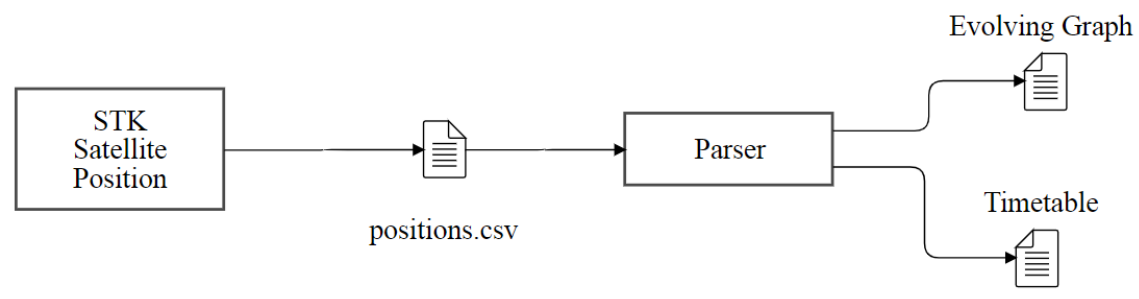

Figure 11: Satellite STK data parsing.

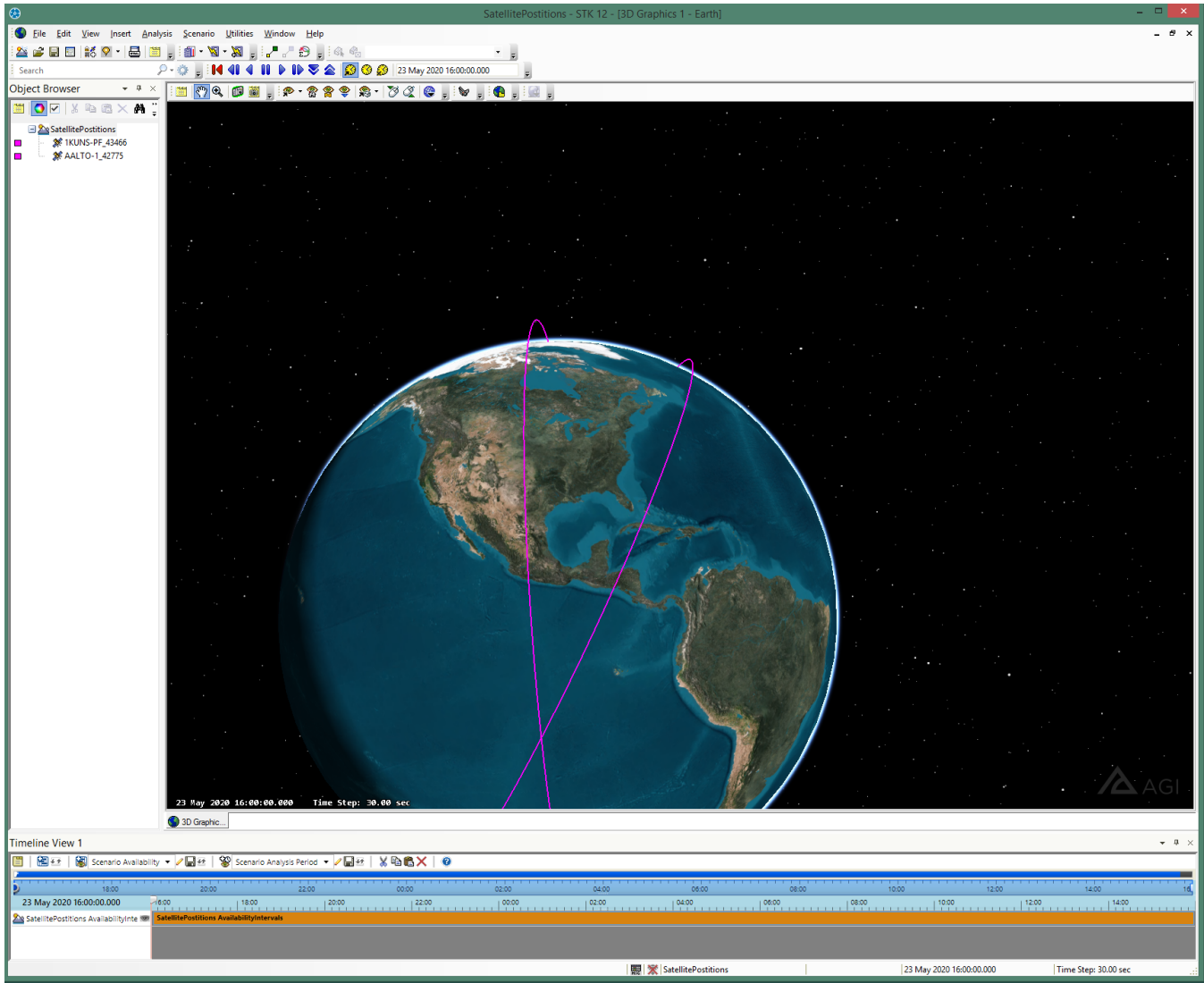

Figure 12: STK screenshot. 
Figure 12 shows an example of how two satellites and their trajectory around the world look like in STK.

We start with 100 satellites and end up with 1000 satellites in 100 steps. One important feature to highlight of this data set is that it has a built-up approach. In other words, the mobility is the same for every set of satellites except for the 100 new added satellites. Regarding the transmission range, it starts with $500 \mathrm{~km}$ and ends up with $6000 \mathrm{~km}$ in $500 \mathrm{~km}$ steps. If we combine each density of nodes and transmission ranges then we have 120 different Evolving Graphs and Timetables to run our experiments. Each experiment chooses 10000 random pairs of source-target satellites and a random $t_{q}$. Thus, they provide 1200000 time measurements for each algorithm. Note that once again, we only include a (source,target) pair, if target is reachable from source.

\subsubsection{STM}

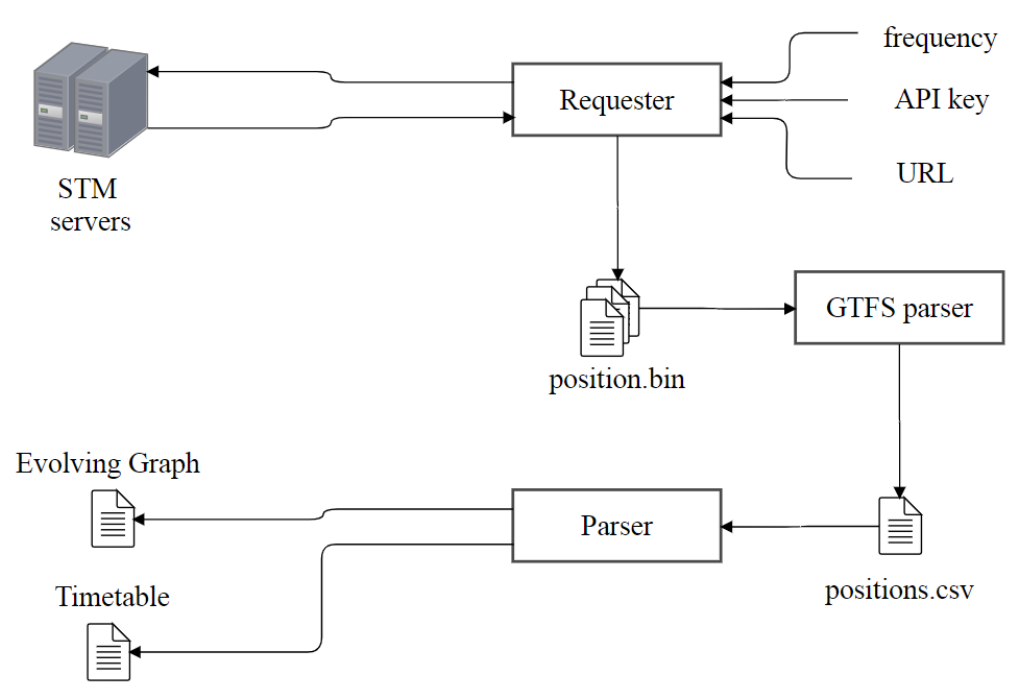

Figure 13: STM data parsing.

STM has a developer API available at https://api.stm.info which makes the bus real-time position publicly available. They use the GTFS Realtime [12] specification to define the position of each bus. GTFS Realtime stands for General Transit Feed Specification that allows public transportation systems to provide real-time updates regarding their transit services. 
We have developed an automatic Requester, as Figure 13 shows, which requires the URL, an API developer key provided by STM, and the frequency. The frequency tells how often the automatic Requester requests the position of the buses to the STM servers. Each response is saved as a position.bin file. After a period of time many position.bin files are collected, we decode the GTFS binary data contained in each file and save all in one single plain-text file called positions.csv. Finally, we calculate the euclidean distance between each pair of buses and create a connection between a pair of nodes in a time step if they are within a given transmission range.

The main characteristic of this data source is that the number of nodes is constant equal to 1152 buses, that is, we use all buses. So, the transmission range is the only variable that we can modify. We start with $150 \mathrm{~m}$ and end up with $2000 \mathrm{~m}$ in $50 \mathrm{~m}$ steps. Thus, we have 38 different Evolving Graphs and Timetables to run our algorithms. Similarly as SUMO and STK data set, each experiment chooses 10000 random pairs of source - target buses and a random $t_{q}$. Therefore, each algorithm has 380000 samples to compare their performance. We only include a (source,target) pair, if target is reachable from source.

\subsection{Characteristics of data sets}

This section describes some characteristics of our data sets, namely: time slot characteristics, number of connections, presence ratio, and dynamic ratio. The time slot characteristics lets us know how many time slots the evolving graph or the timetable has and how long each time slot lasts in real time. The number of connections as its name suggest tells the size of the timetable. This attribute is useful in terms of order of magnitude.

We define two metrics in the following sections called presence ratio and dynamic ratio. Basically, presence ratio is a comparison between the dynamic graph and its static graph equivalent. Dynamic ratio is a comparison between the actual number of on-off transitions of each edge and its maximum possible value of on-off transitions.

\subsubsection{Time slots}

Table 1 summarizes the main characteristics regarding time slots. The slot duration means the real elapsed time between each time step. The total duration refers to the 


\begin{tabular}{|cccccc|}
\hline $\begin{array}{c}\text { Data } \\
\text { Set }\end{array}$ & $\begin{array}{c}\text { Mobility } \\
\text { Nature }\end{array}$ & $\begin{array}{c}\text { Max-Min } \\
\text { Connections }\end{array}$ & $\begin{array}{c}\text { Number of } \\
\text { Time Slots }\end{array}$ & $\begin{array}{c}\text { Slot } \\
\text { Duration }\end{array}$ & $\begin{array}{c}\text { Total } \\
\text { Duration }\end{array}$ \\
\hline SUMO & random & $92007-209$ & 120 & 1 second & 120 seconds \\
\hline STK & real & $2278948-1048$ & 194 & 7.5 minutes & 24 hours \\
\hline STM & real & $317302-51300$ & 1684 & 12.8 seconds & 6 hours \\
\hline
\end{tabular}

Table 1: Time slots.

whole duration of simulation or data collection. In other words, the total duration is equal to slot duration times the number of time slots. STM data set is the one with the greatest number of time steps while STK has the biggest slot duration around 7.5 minutes.

\subsubsection{Number of connections}

Figures 14, 16 and 17 show the average number of connections for every data set. As expected, the number of connections grows as we increase either the number of nodes or the transmission range.

The slope for SUMO and STM data sets in Figures 17b and 14b is constant as we increase the transmission range, however in Figure 16b the slope of STK data set grows faster at the beginning but then it tends to decrease as the transmission range increases. This is because the transmission range has a limit. In other words, there is a point where the transmission is so big that the network is completely connected. Figure 15 shows an evidence of our previous statement. It is an experiment in SUMO data set which its transmission range limit is around $6500 \mathrm{~m}$. As we can see, after $6000 \mathrm{~m}$ there is no more new connections since every node is connected to every other node in the network.

Figures 14a, 16a and 17a show how the number of connections increases as we increase the number of nodes.

\subsubsection{Presence ratio}

In order to know how present the edges are in an Evolving Graph $\mathcal{G}$ we compare the average degree of every node during the lifespan of $\mathcal{G}$ with the number of neighbors. Equation 6 shows how we calculate the presence ratio; $s$ is the number of time steps, 


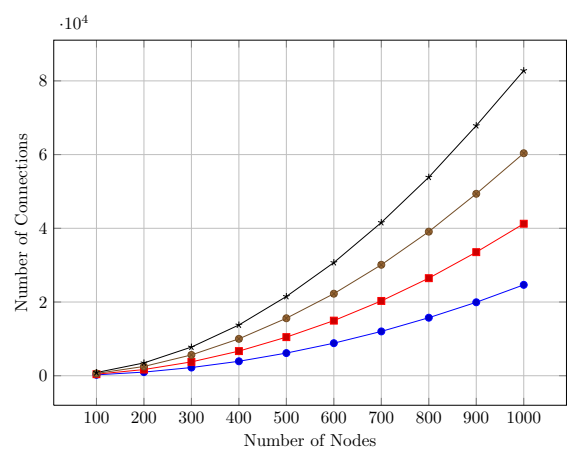

(a) Varying the number of nodes.

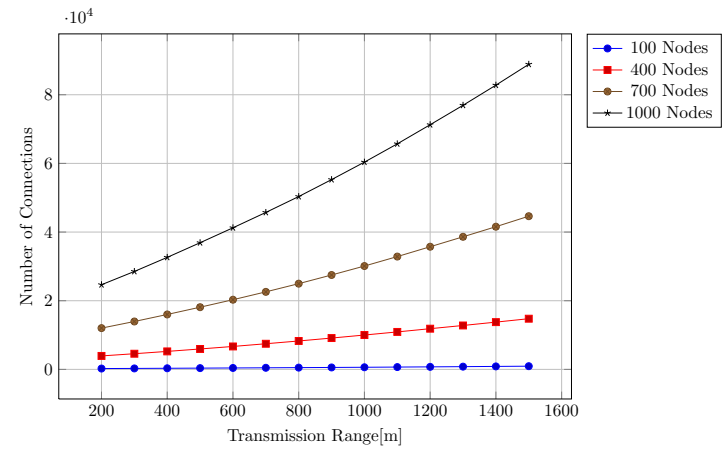

(b) Varying the transmission range

Figure 14: SUMO number of connections.

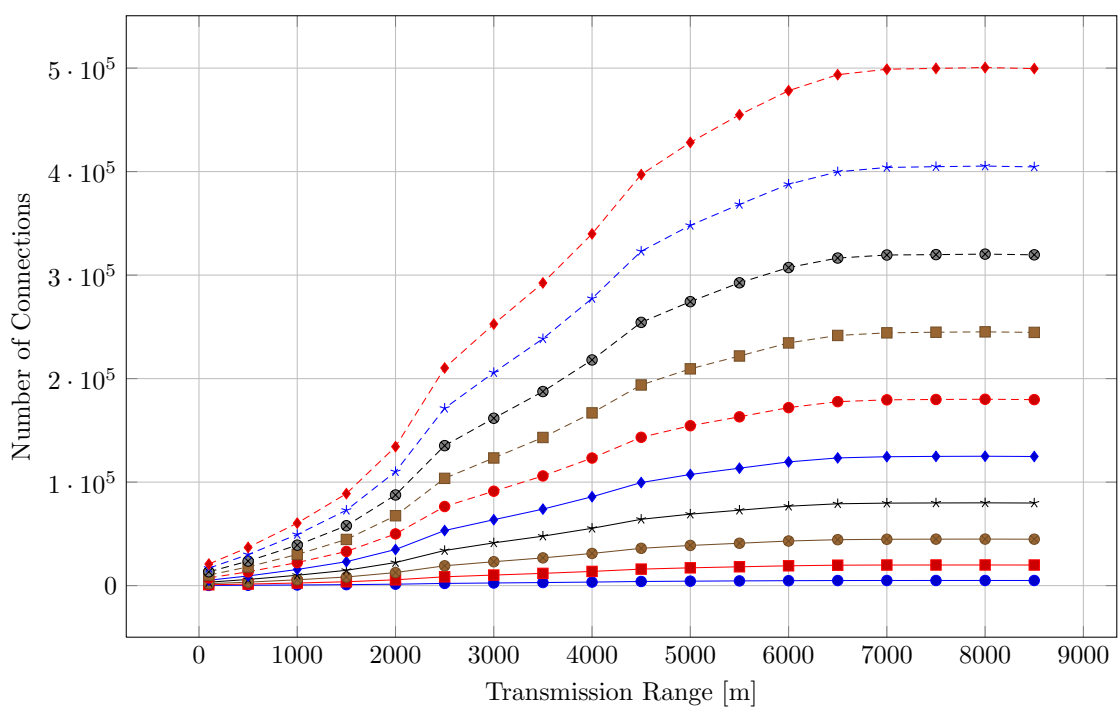

$$
\begin{aligned}
& \text { - } 100 \text { Nodes } \\
& \text { - } 200 \text { Nodes } \\
& \text { - } 300 \text { Nodes } \\
& \text { - } 400 \text { Nodes } \\
& \text { - } 500 \text { Nodes } \\
& \text { - - } 600 \text { Nodes } \\
& \text { - } 700 \text { Nodes } \\
& \text { - }-800 \text { Nodes } \\
& \text { - - } 900 \text { Nodes } \\
& -\leftarrow 1000 \text { Nodes }
\end{aligned}
$$

Figure 15: Number of connections in SUMO data set as the transmission range reaches its limit.

degree $(v, t)$ is the degree of node $v$ at time $t$ while degree $(v)$ is the number of neighbors. For example, lets consider the dynamic graph of Figure 18 where every edge is present for two time steps. If we calculate the presence ratio we have Equation 7 which shows that it is equal to 0.20 . So in average the edges are present $20 \%$ of the time. So, if the presence ratio is equal to one then it means that every edge is present 


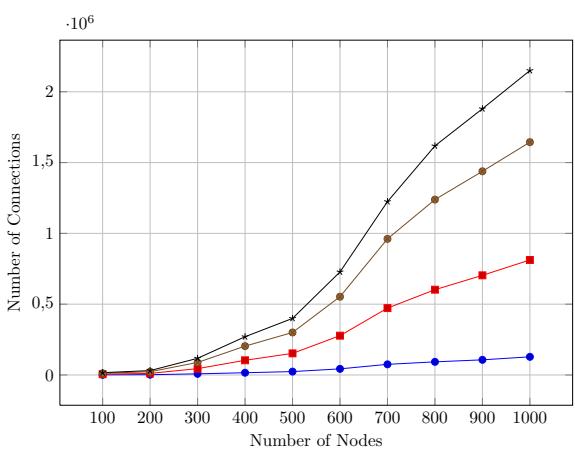

(a) Varying the number of nodes.

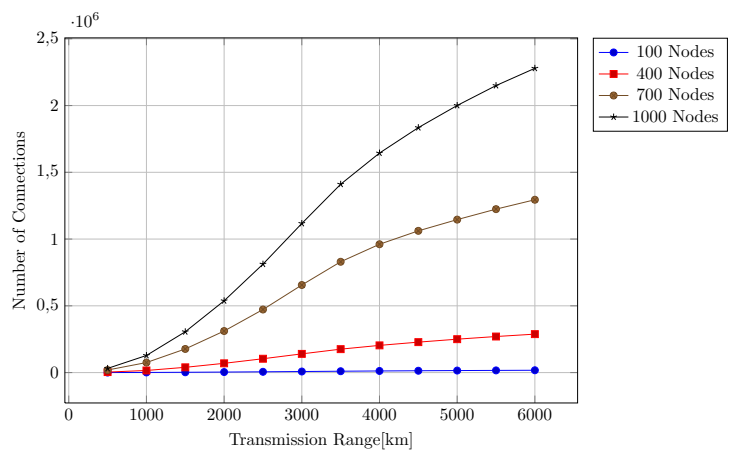

(b) Varying the transmission range

Figure 16: STK number of connections.

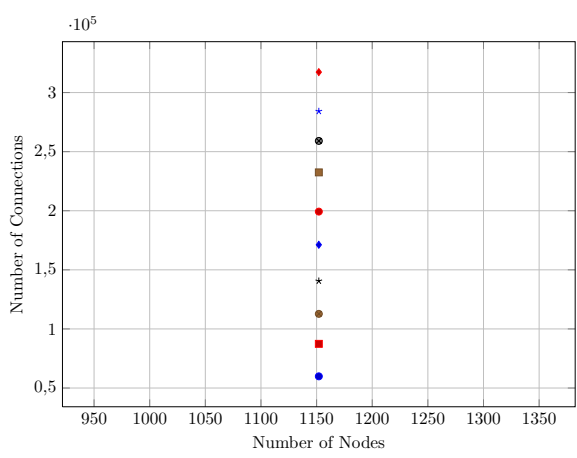

(a) 1152 nodes

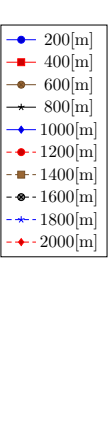

$400[\mathrm{~m}]$

$-600[\mathrm{~m}]$
$-800[\mathrm{~m}]$

$-1000[\mathrm{~m}]$

$-1400[\mathrm{~m}]$

$-1600[\mathrm{~m}]$

$-2000[\mathrm{~m}]$

Figure 17: STM number of connections.

all the time in $\mathcal{G}$.

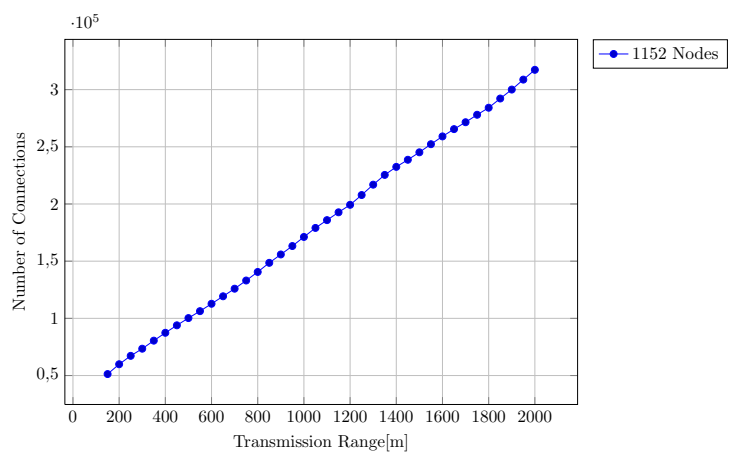

(b) Varying the transmission range

$$
\begin{aligned}
\text { presenceRatio }(\mathcal{G})=\frac{\sum_{v \in V} \sum_{i=0}^{s} \operatorname{degree}\left(v, t_{i}\right)}{s * \sum_{v \in V} \operatorname{degree}(v)} \\
=\frac{\sum_{i=0}^{10} \operatorname{degree}(A, i)+\sum_{i=0}^{10} \operatorname{degree}(B, i)+\sum_{i=0}^{10} \operatorname{degree}(C, i)+\sum_{i=0}^{10} \operatorname{degree}(D, i)}{10 *(\text { degree }(A)+\operatorname{degree}(B)+\operatorname{degree}(C)+\operatorname{degree}(D))} \\
=\frac{20}{10 *(2+3+3+2)} \\
=\frac{1}{10 * 10}
\end{aligned}
$$




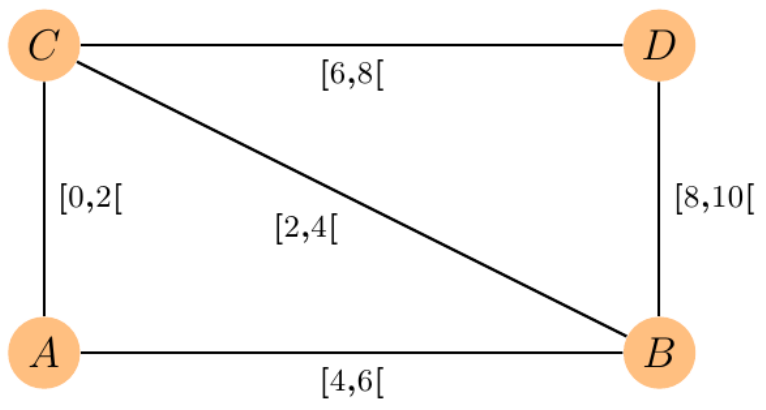

Figure 18: Presence ratio example.

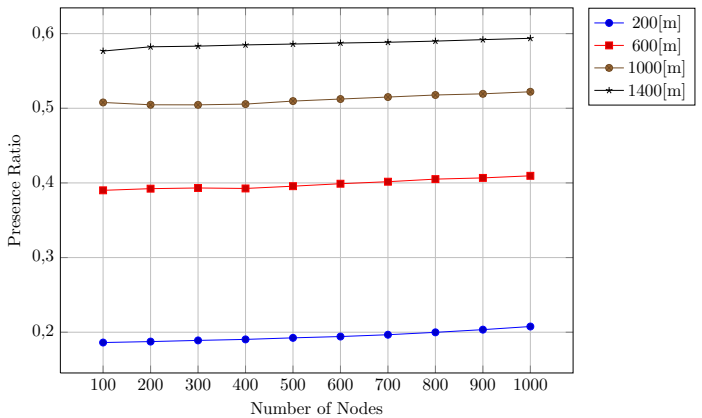

(a) Varying the number of nodes

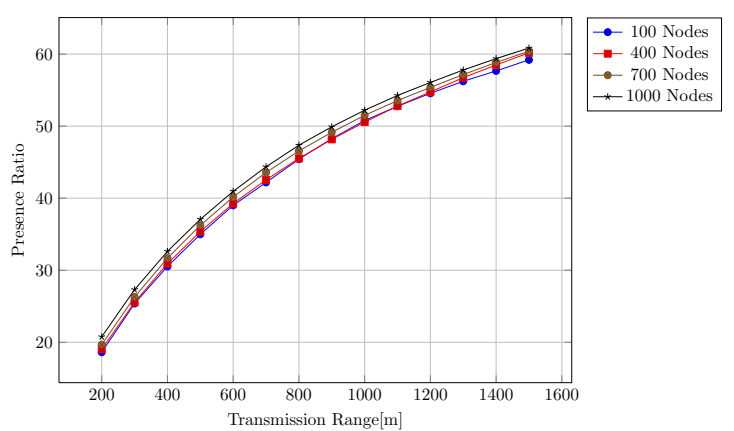

(b) Varying the transmission range

Figure 19: SUMO presence ratio.

Figures 19, 20 and 21 show how the presence ratio varies as we increase the transmission range or the number of nodes. For the SUMO data set, increasing the number of nodes for a given transmission range does not impact the presence ratio as increasing the transmission range for a given density of nodes does. Figure 19b shows that for smaller transmission ranges the slop is high and it tends to decrease as the transmission range increases. This behavior can be described as some sort of saturation since the slope grows slower as we are getting close to presence ratio equal one. The maximum values in Figures 19a and 19b are around 0.6 which means that the edges are present on average sixty percent of the time.

Figure 20a shows that STK data set has the maximum presence ratio at 200 nodes for every transmission range. After the curve reaches this peak; it decreases and then tends to be constant as we increase the number of nodes. If we take a closer look at Figure 20a then we realize that if we increase the number of nodes for a given transmission range then the presence ratio does not change. In Figures 20b and 21b, we see constant slope; as transmission range increases, the presence ratio increases 


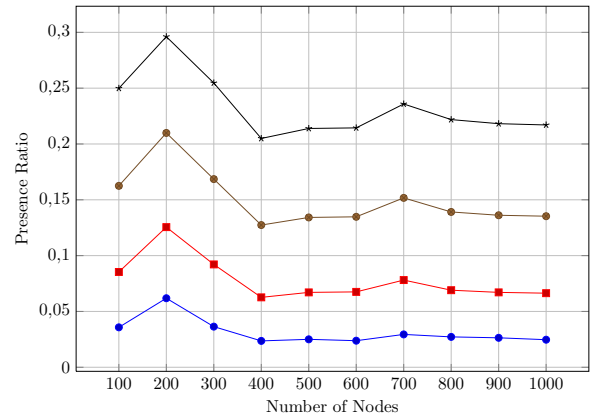

(a) Varying the number of nodes

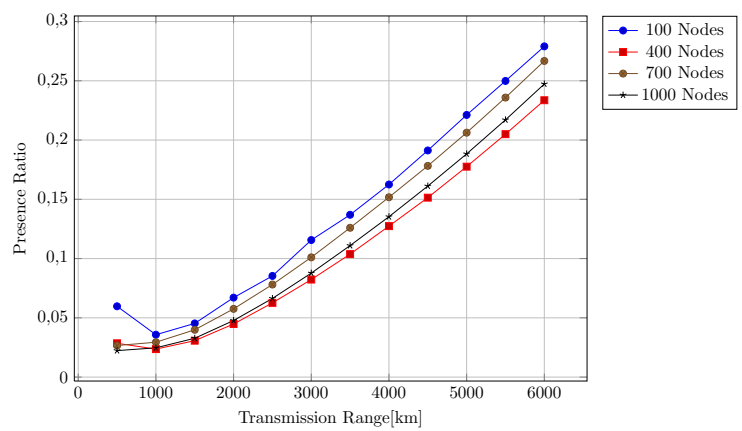

(b) Varying the transmission range

Figure 20: STK presence ratio.

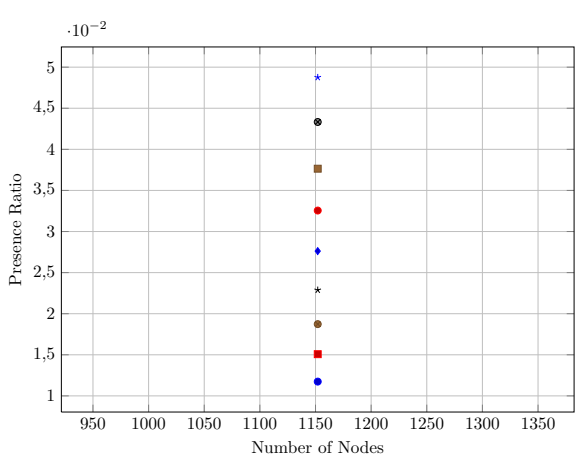

(a) 1152 nodes

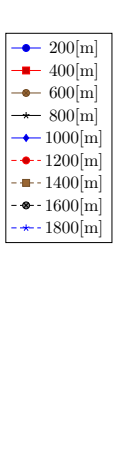

$-200[\mathrm{~m}]$
$-400[\mathrm{~m}]$
$-600[\mathrm{~m}]$
$-800[\mathrm{~m}]$
$-1000[\mathrm{~m}]$
$-1200[\mathrm{~m}]$
$-1400[\mathrm{~m}]$
$-1600[\mathrm{~m}]$
$-1800[\mathrm{~m}]$

$1800[\mathrm{~m}]$

Figure 21: STM presence ratio.

linearly. Since the space where STK and STM are running is big compared to SUMO we do not saturate the presence ratio as fast as we do for SUMO.

\subsubsection{Dynamic ratio}

The presence ratio metric gives us an overall measure on how present the edges are in $\mathcal{G}$ but there is no information about their dynamicity. In other words, we want to know in average how many intervals the edges have. For example, let's consider an edge between node $v$ and node $a$ in $\mathcal{G}$ with the following schedule: [0, $1[,[2,3[,[4,5[$. Figure 22 shows the plot of the previous schedule. This type of schedule is considered as highly dynamic because it has the highest frequency switching between active and non-active at every time step. In the other hand, the lowest frequency is shown in Figure 23 which has only one interval.

If we compare the plots of Figures 22 and 24; we find out that they have the same 


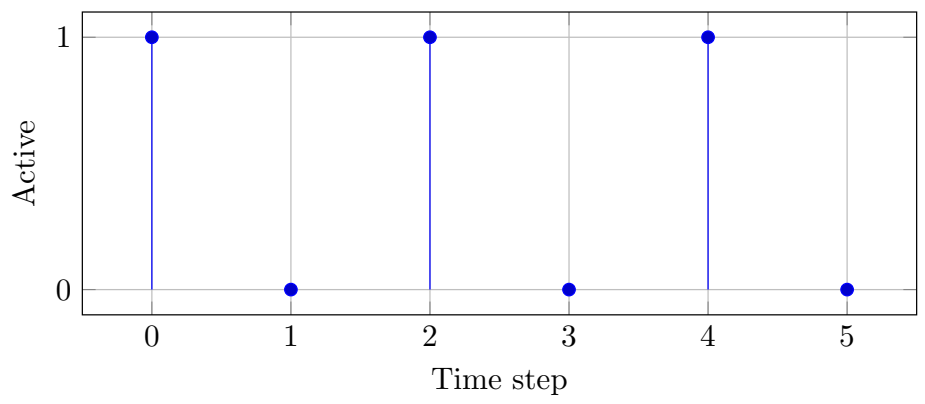

Figure 22: Schedule with intervals switching between active and non-active.

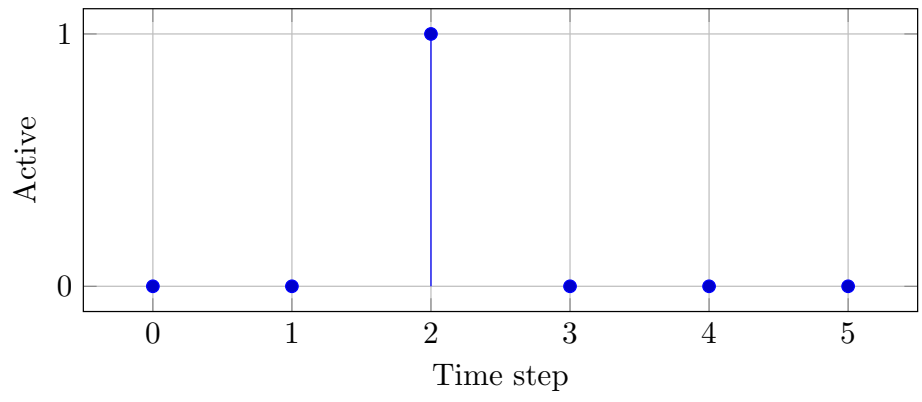

Figure 23: Schedule with only one interval.

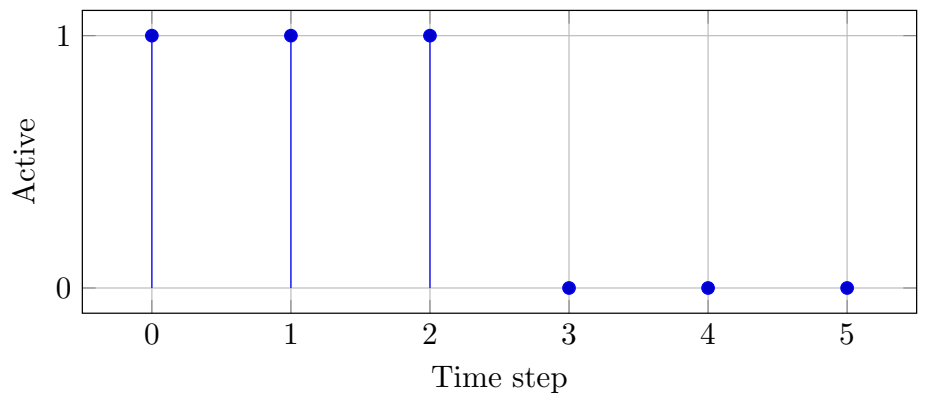

Figure 24: Schedule with one interval with three consecutive time steps. 


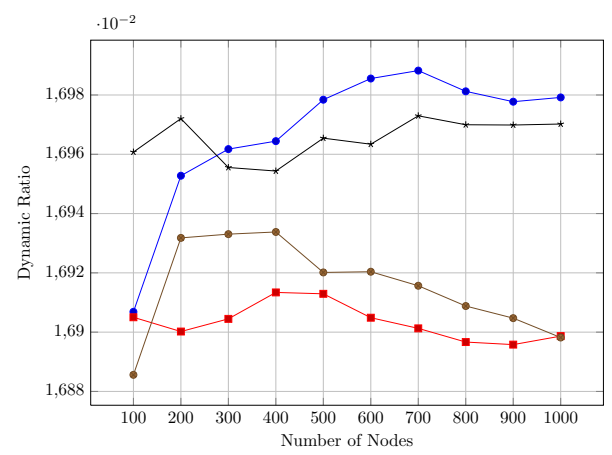

(a) Varying the number of nodes

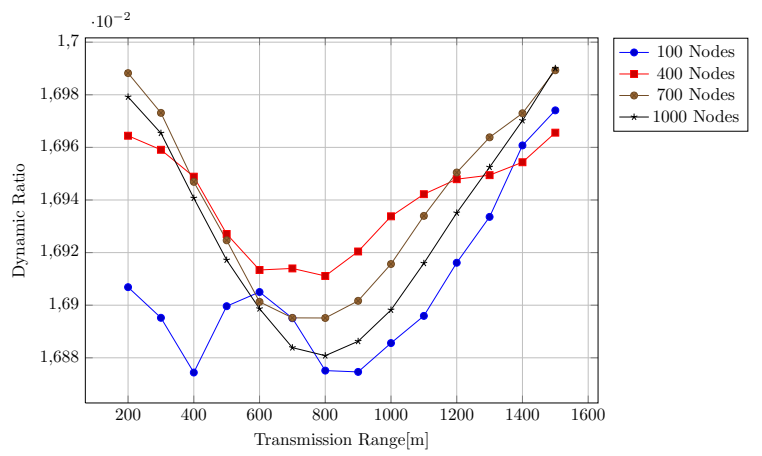

(b) Varying the transmission range

Figure 25: SUMO dynamic ratio.

presence ratio equal to 0.5 but Figure 24 is less dynamic because it has only one transition between active and non-active.

$$
\begin{aligned}
\operatorname{dynamicRatio}(\mathcal{G}) & =\frac{\sum_{v \in V} \frac{\sum_{a \in \text { neighbors }(v)} \operatorname{scheduleSize}(v, a)}{\operatorname{degree}(v)}}{|V| * \frac{s+1}{2}} \\
& =\frac{2 * \sum_{v \in V} \frac{\sum_{a \in \text { neighbors }(v)} \operatorname{scheduleSize}(v, a)}{\operatorname{degree}(v)}}{|V| *(s+1)}
\end{aligned}
$$

Equation 8 shows the summation of the average number of intervals of every node in $\mathcal{G}$ divided by the maximum frequency of every schedule. scheduleSize $(v, a)$ returns the number of intervals in the edge $(v, a)$. degree $(v)$ is the number of neighbors of $v$. $|V|$ is the number of nodes in $V$ and $s$ is the number of time steps.

The numerator of Equation 8 can be equal to the denominator only if every neighbor of every node has a schedule with $(s+1) / 2$ intervals which is the maximum possible frequency. Therefore, dynamicRatio $(\mathcal{G})=1$ means that $\mathcal{G}$ has nodes with neighbors highly dynamic switching at every time step.

Figures 25, 27, 28 show how the dynamic ratio behaves according to the variation of the number of nodes or transmission range. SUMO data set displays in Figure 25 a very tiny variations as the number of nodes increases for a given transmission range. This behaviour shows that an increment in the number of nodes does not have 


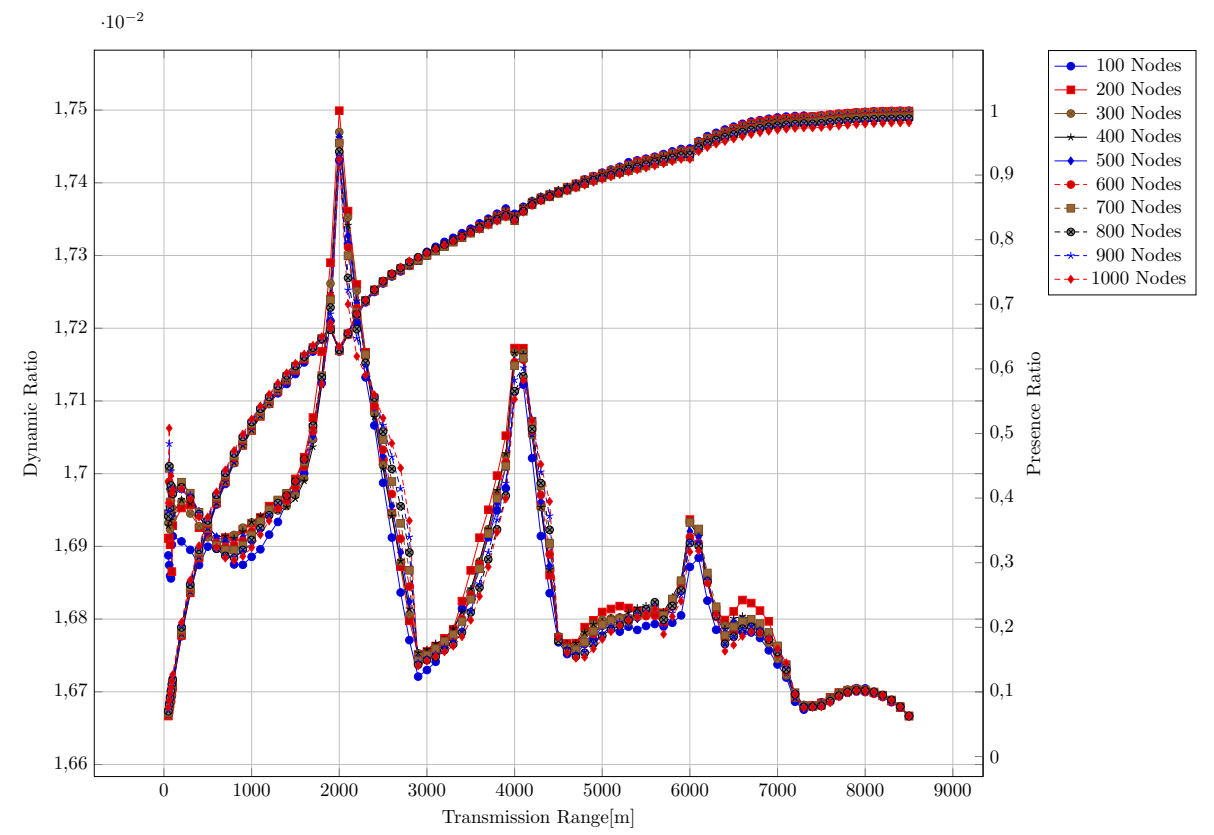

Figure 26: SUMO comparison between presence ratio and dynamic ratio.

a significant impact in the dynamic ratio since it measures the number of intervals in each schedule. In other words, it is not about the number of neighbors but the times they connect and disconnect. The transmission range provides more stable connections if we increment its value as we can see in Figure 25b. But, around 800m, nodes tend to have more intervals again. Thus, an increment in the transmission range causes more stable connections but if the increment is high enough it will add new neighbors that connect and disconnect more often. To show an evidence of our previous reasoning, for SUMO data set we increase the transmission range up to $8500 \mathrm{~m}$ to see how the dynamic ratio behaves.

Figure 26 shows two plots; the first one is the dynamic ratio at the left axis while the second plot shows the presence ratio at the right axis. The presence ratio seems to have a logarithmic behaviour as we increase the transmission range. We also see the same concave curve that we see in Figure 25b for the first transmission ranges. At $2000 \mathrm{~m}$ the network experiences a significant increment in its dynamic ratio meaning that there are more neighbors that connect and disconnect more often. Then, at 3000m the edges become stable with less intervals. Further transmission ranges provokes more peaks but less pronounced until it fades reaching its possible minimum value around 0.0166 . Notice that at $2000 \mathrm{~m}, 4000 \mathrm{~m}$ and $6000 \mathrm{~m}$ when the 


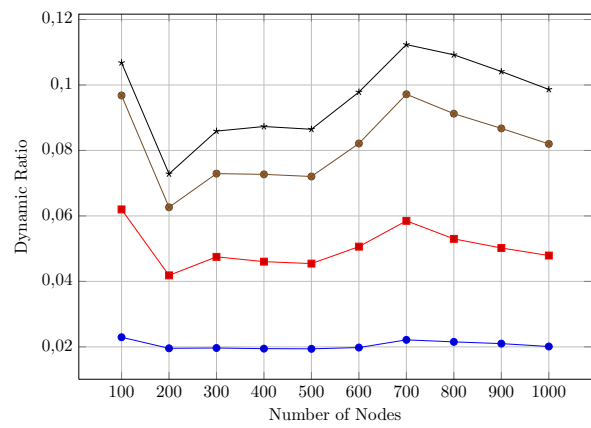

(a) Varying the number of nodes

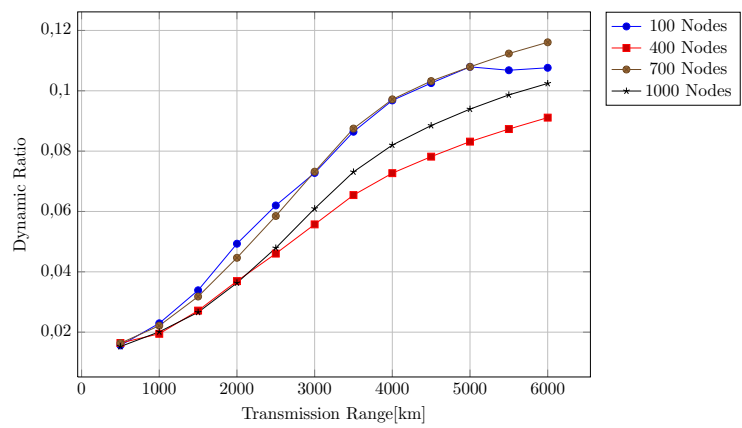

(b) Varying the transmission range

Figure 27: STK dynamic ratio.

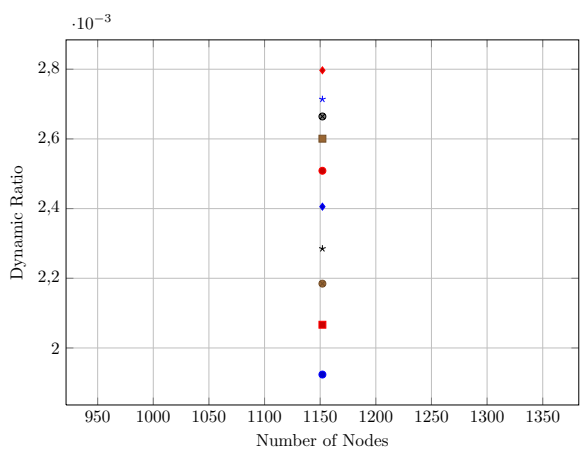

(a) 1152 nodes

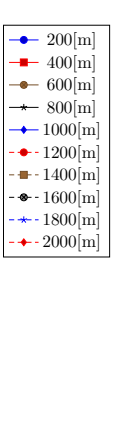

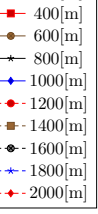

Figure 28: STM dynamic ratio.

network has a peak in the dynamic ratio the network experiences a small interruption in the presence ratio along its smooth tendency.

Figure 27a shows that for STK data set the dynamic ratio is constant for the first transmission ranges. For larger transmission ranges, the curves tend to have a low peak at 200 nodes and a high peak at 700 nodes. If we look closely to the same density of nodes in Figure 20a; we find out that the network at 200 Nodes is experiencing the same phenomenon that we described earlier for SUMO. For the same density of nodes when there is a peak in the dynamic ratio there is low point in the presence ratio. But, for 700 nodes we have peaks in both ratios. This means that the new added edges because of the increment of the transmission range are not disconnecting that often.

The dynamic ratio for STM data set in Figure 28b has the same shape of presence ratio in Figure 21b. It is showing that an increment in the transmission range provokes more present intervals. 


\section{Chapter 5}

\section{Experimental results}

In this chapter we analyse the performance of the algorithms but first in Section 5.1 we analyse the characteristics of the computed journeys considering the average journey duration and the average of distinct journey durations. Finally, in Section 5.2, we examine and compare in terms of running time and number of hops the performance of our timetable-based algorithms against Ferreira's algorithm. We study how the algorithms behave by modifying either the transmission range or the number of nodes. We use the Timer Boost $\mathrm{C}++[46]$ library to measure the running time.

\subsection{Computed journey characteristics analysis}

We subtract the arrival time or $d($ target $)$ from the query time $t_{q}$ to have the travel time or journey duration. If the duration is equal to zero then the target node is reachable instantaneously from the source node. The number of distinct journey durations/arrival times is computed by finding the number of different $T\left(s, t_{q}, t\right)$ sets. This gives us a more qualitative idea of how much time is spent waiting. For example, suppose there are only two distinct journey durations, 0 and $\mathcal{T}_{\text {end }}-t_{q}$, then clearly at least for one of the possible destinations, a packet has to wait a long time before being transmitted. On the other hand, if there are $\mathcal{T}_{\text {end }}-t_{q}$ distinct journey durations possible, then it is likely that for many journeys, packets don't spend too much time waiting to be transmitted. The previous variables gives us a sense about the temporal properties of the journey but there is no information regarding its physical properties. This is why we consider and plot the number of hops in the next section. For instance, 


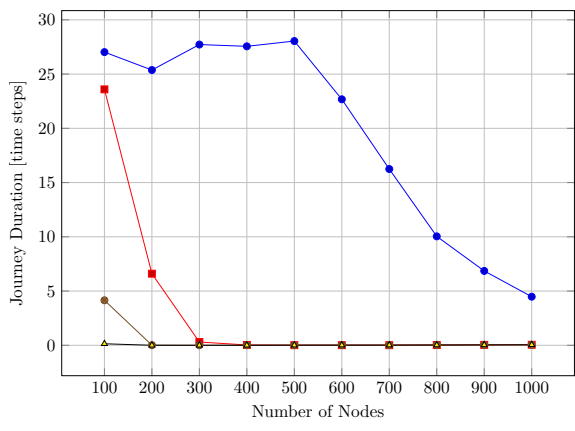

(a) Journey duration.

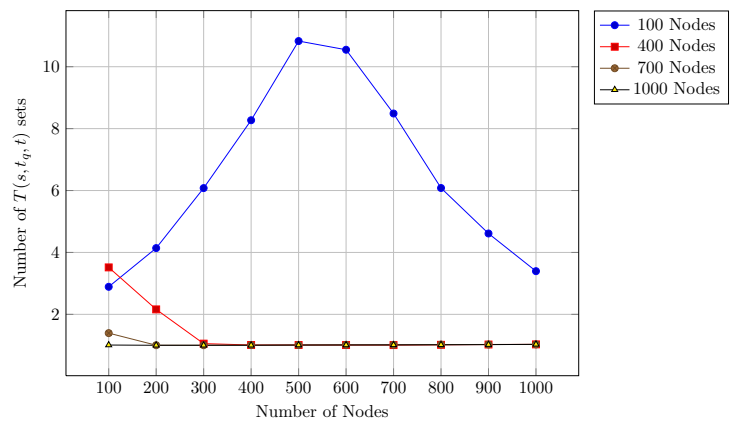

(b) Number of journey durations.

Figure 29: SUMO varying the number of nodes.

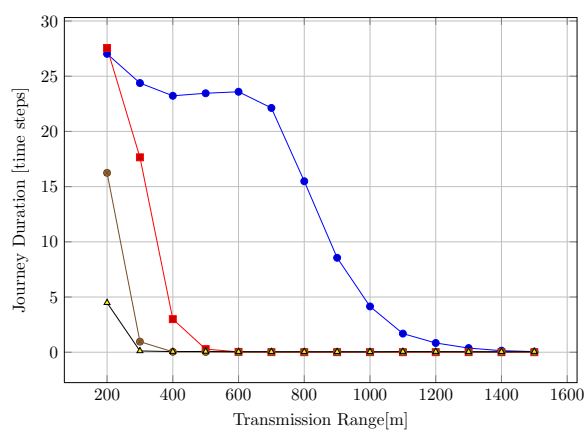

(a) Journey duration.

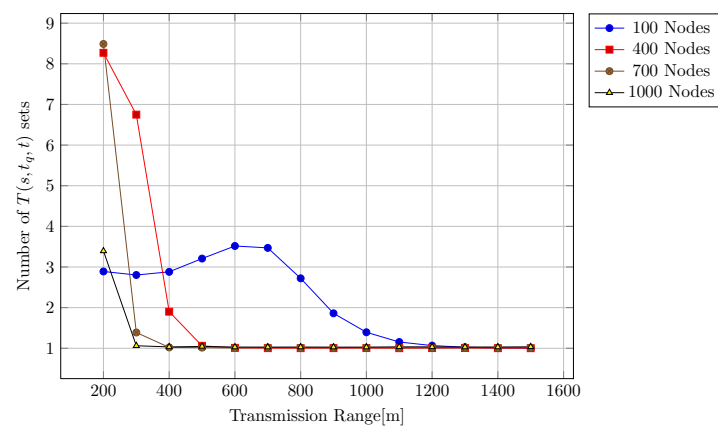

(b) Number of journey durations.

Figure 30: SUMO varying the transmission range.

it may be possible to have an instantaneous journey $d($ target $)-t_{q}=0$ but with $|V|-1$ hops.

\subsubsection{SUMO}

We proceed to analyze SUMO journeys data set by fixing the transmission range and modifying the number of nodes. Figures 29a and 29b, show the average journey duration, and number of distinct journey durations respectively. As we can see, there is a maximum value for the average journey duration around 27 time steps for $200 \mathrm{~m}$ transmission range with 500 nodes. We also have the maximum number of $T$ sets at the same coordinate. We appreciate in Figure 29b the same number of $T$ sets for different density of nodes, for instance 100 and 1000 nodes. But comparing it with Figure 29a then we find out that for 100 nodes the packet is on hold for many time steps while for 100 nodes the journey tends to be instantaneous.

Figures 30a and 30b show the variables described before but fixing the number of 
nodes and varying the transmission range. There is longer journey duration for lower transmission ranges which means that the packet is on hold at some nodes while for longer transmission ranges the journey tends to be instantaneous. The number of $T$ sets is high for lower transmission ranges and it tends to decrease as the transmission range increases.

Overall, we conclude that for the SUMO data set, the journey duration decreases as the number of nodes (i.e. density of nodes) increases, or as the transmission range (i.e. density of edges) increases.

\subsubsection{STK}

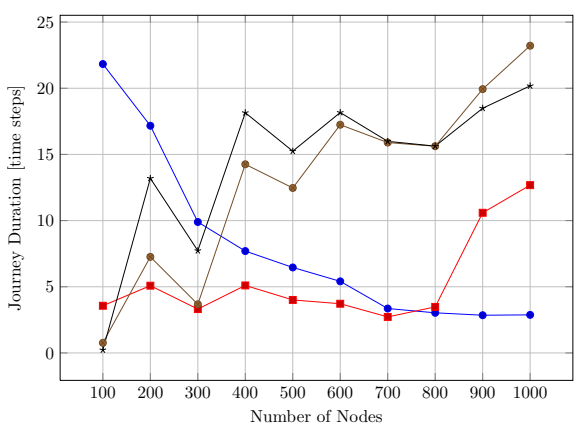

(a) Journey duration.

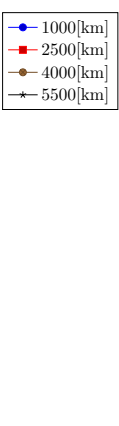

Figure 31: STK varying the number of nodes.

We perform the same analysis for STK data set. If we compare Figures 31a and $31 \mathrm{~b}$ the we find out that both plots tend to have the same shape. For instance, for a fixed transmission range of $1000 \mathrm{~km}$ the journey duration and the number of $T$ sets

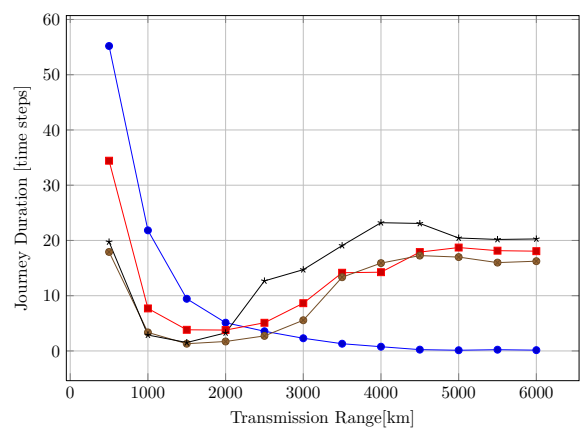

(a) Journey duration.

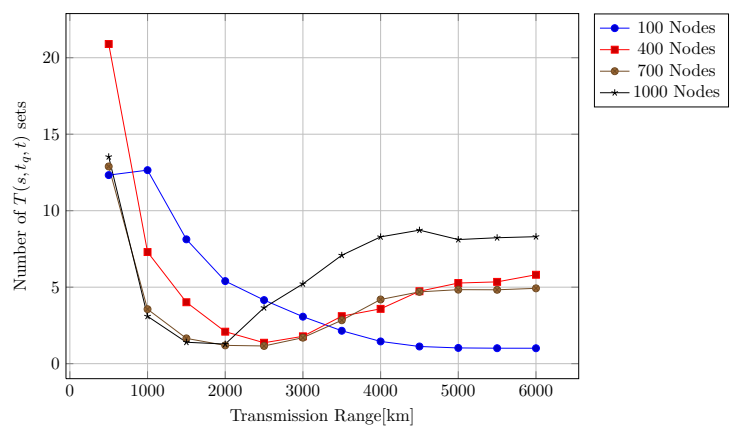

(b) Number of journey durations.

Figure 32: STK varying the transission range. 
have the same decreasing tendency. This means that the packet is not on hold for many time steps. The same happens for $4000 \mathrm{~km}$ and $5500 \mathrm{~km}$ but with the opposite tendency; the packet tends to travel more but without holding much.

Figures $32 \mathrm{a}, 32 \mathrm{~b}$ show the effect of varying the transmission range with a constant number of nodes in the journey characteristics. An increment in the transmission range for lower number of nodes provokes less journey duration while for higher number of nodes the journey duration reaches a lower point and tends to increase.

\subsubsection{STM}

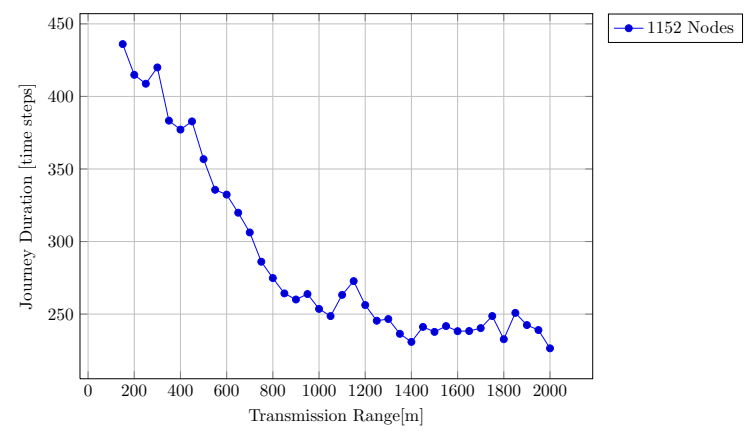

(a) Journey duration.

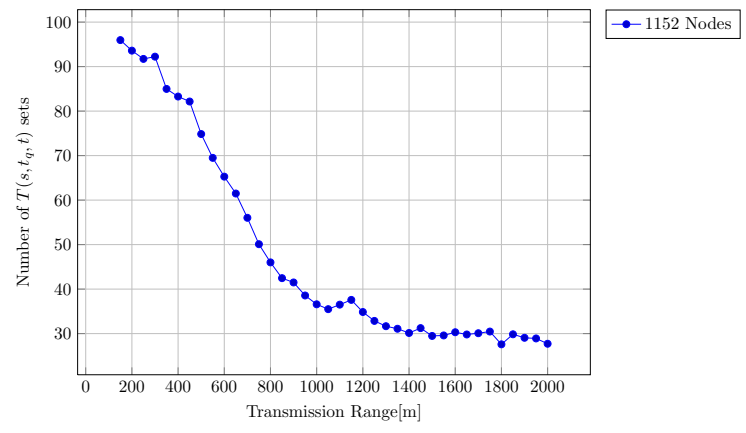

(b) Number of journey durations.

Figure 33: STM varying the transmission range.

Figures 33a, 33b show the behavior of the journey durations. The range for the transmission range that we have for STM data set is wider. This data set has a lot more time slots than the previous data sets; this is why for the first transmission range $150 \mathrm{~m}$ we have a journey duration around 450 time steps along with almost one hundred $T$ sets. An increment in the transmission range originates a reduction in both variables.

\subsection{Performance analysis}

This section studies the performance of our algorithms in terms of running time. Since a sequence of connections can be traversed instantaneously, different journeys with the same duration can have different numbers of hops. So we also compare the number of hops in journeys produced by the different algorithms. As we did 
previously, we fix either the number of nodes or the transmission range and vary the non-fixed variable to measure the running time.

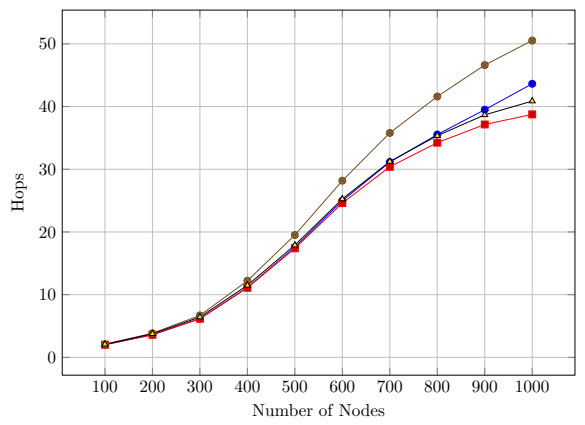

(a) $200[\mathrm{~m}]$

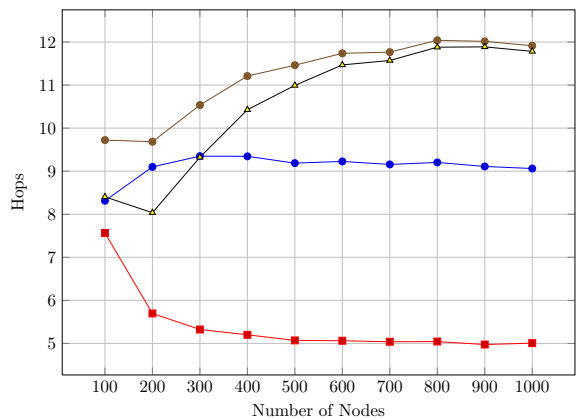

(c) $1000[\mathrm{~m}]$
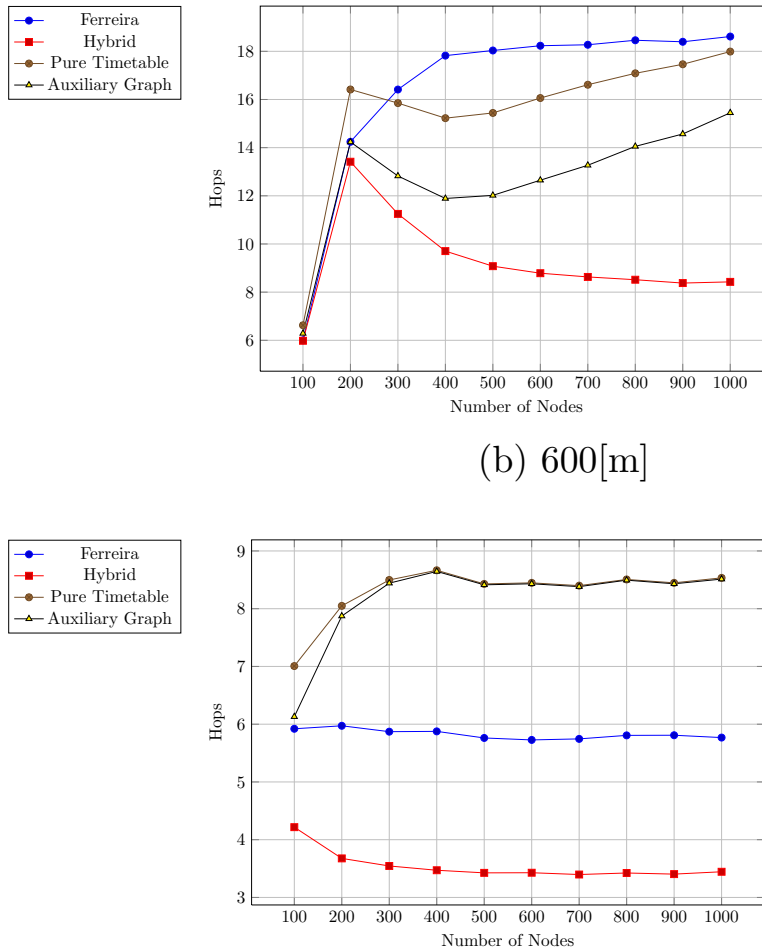

(d) $1400[\mathrm{~m}]$

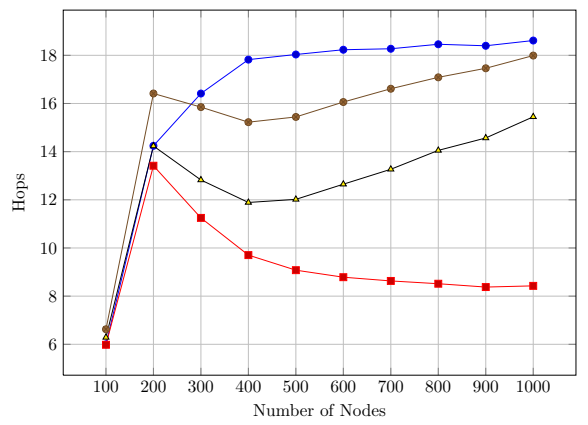

(b) $600[\mathrm{~m}]$
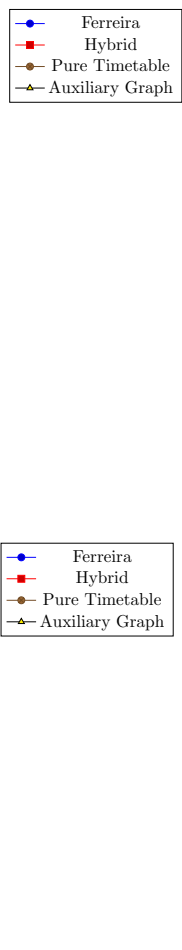

Figure 34: SUMO number of hops varying the number of nodes.

\subsubsection{Effect of varying the number of nodes}

In this section, we keep the transmission range fixed, and study the effect of varying the number of nodes. As stated earlier, for the STM data set, we only have one number of nodes, so the experiments in this section are only for the SUMO and STK data sets.

\section{SUMO: Number of hops}

Figures 34a to 34d show the effect of varying the number of nodes for fixed transmission range on the number of hops in the foremost journeys produced by the four algorithms we study. There are two things to note here. The first is that even though all algorithms compute journeys with the same arrival time for a given input, their behavior in terms of number of hops in the journey is quite different, especially at 
higher transmission ranges. We see that Hybrid Timetable-Evolving Graph always finds paths with the fewest number of hops, while Pure-Timetable appears to find the journey with the highest number hops.

The second thing to note is that for low transmission range, the average number of hops increases with increasing number of nodes for all algorithms, while for higher transmission ranges, the number of hops does not appear to be affected by the number of nodes in the network, once there are enough nodes in the network. A likely explanation is that at low transmission ranges, nodes that are far apart are unreachable, and therefore discarded, so when the number of nodes is small, then they are close by in the network, and the foremost journey has few hops. As the number of nodes grows, we are able to reach nodes at greater distance, and greater number of hops. For higher transmission ranges, as seen in Figure 29, the journey duration is zero or close to zero, and all nodes are essentially reachable immediately, so that the number of hops does not change with increased number of nodes.

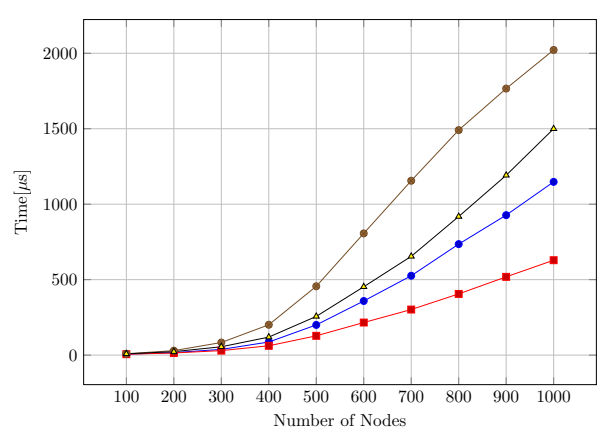

(a) $200[\mathrm{~m}]$

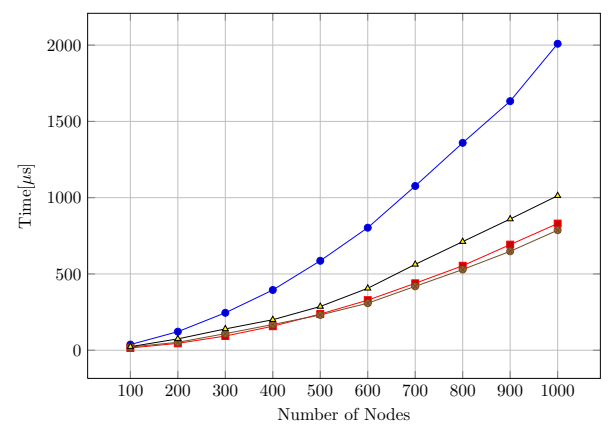

(c) $1000[\mathrm{~m}]$
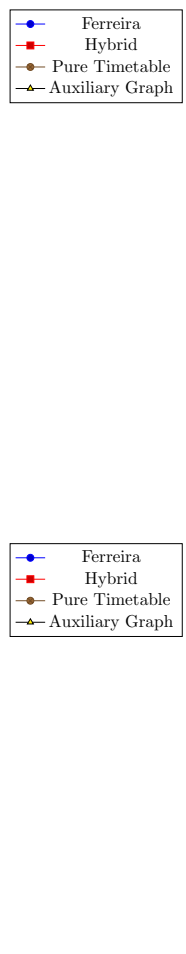

Figure 35: SUMO running time varying the number of nodes.

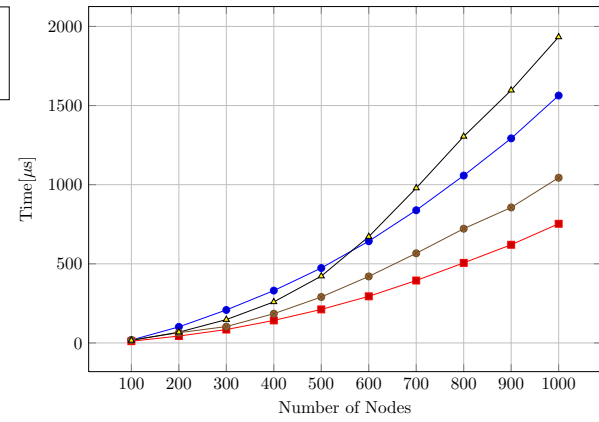

(b) $600[\mathrm{~m}]$
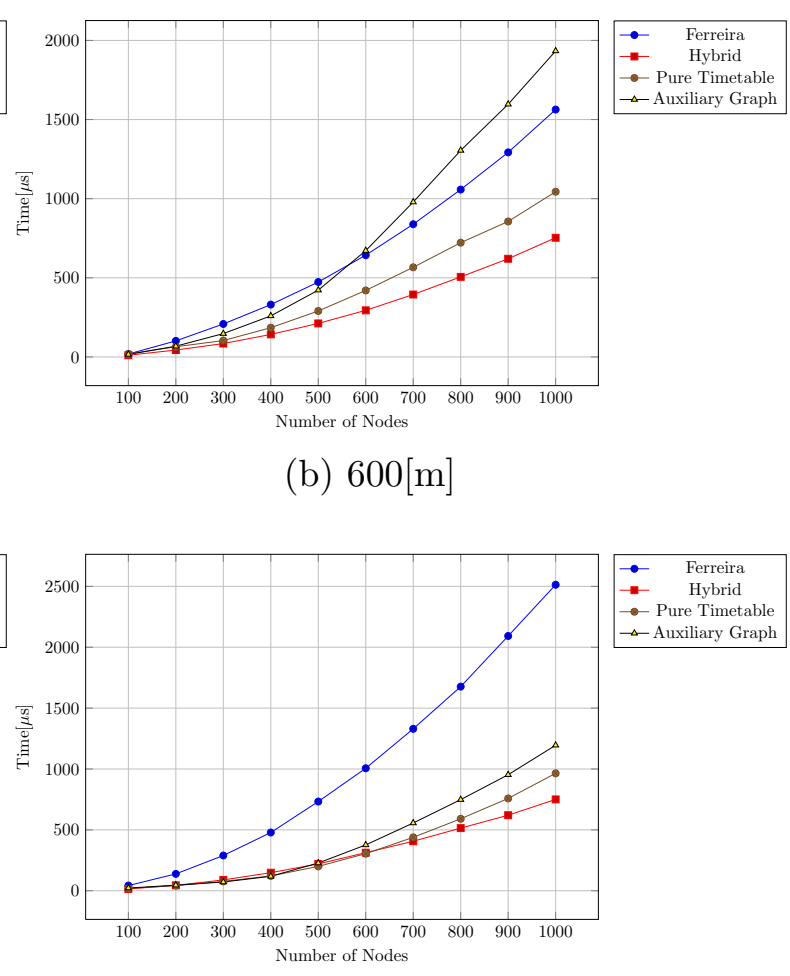

(d) $1400[\mathrm{~m}]$ 


\section{SUMO: Running time}

Figures 35 a to $35 \mathrm{~d}$ show the running time of all 4 algorithms for networks with transmission range $200 \mathrm{~m}, 600 \mathrm{~m}, 1000 \mathrm{~m}$ and $1400 \mathrm{~m}$. As expected, for each of those transmission ranges, the running time of all the algorithms increases with increasing number of nodes. We see that in all cases, and nearly all densities, Hybrid TimetableEvolving Graph has the best running time. While for smaller transmission range, the Pure-Timetable and Timetable with Auxiliary Graph algorithms are worse than Ferriera's algorithm, for larger transmission ranges, the running time of Ferreira is the worst, and grows faster with the number of nodes than the other algorithms.

\section{STK: Number of hops}

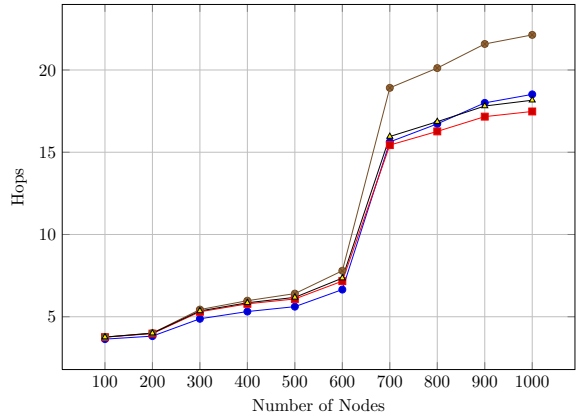

(a) $1000[\mathrm{~km}]$

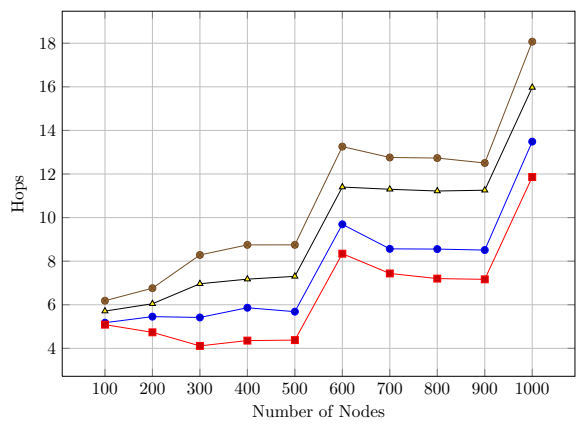

(c) $4000[\mathrm{~km}]$

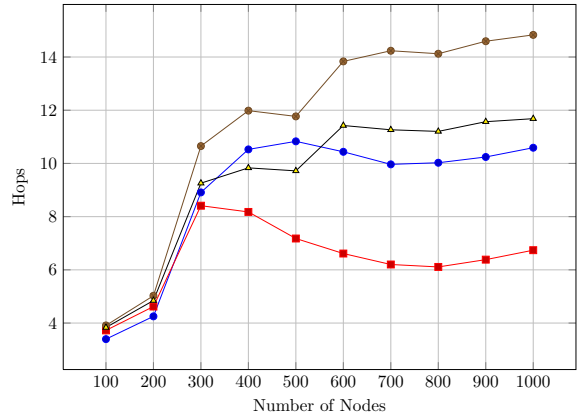

(b) $2500[\mathrm{~km}]$
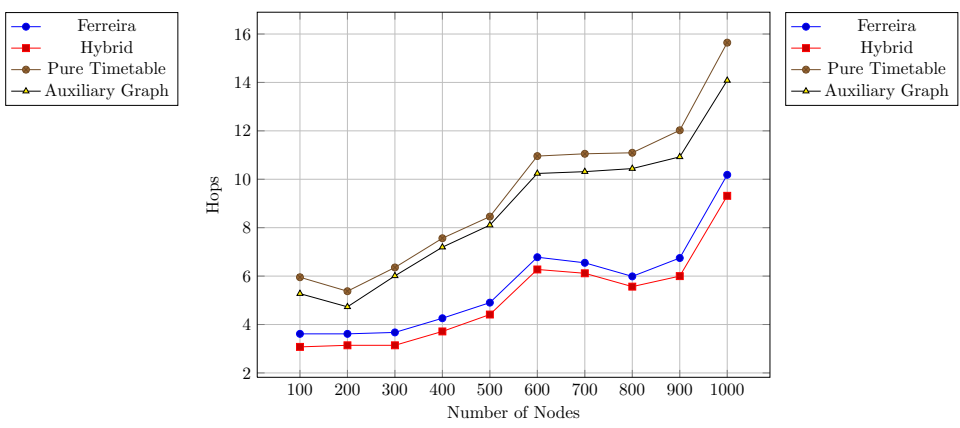

(d) $5500[\mathrm{~km}]$

Figure 36: STK number of hops varying the number of nodes.

We see a sudden increment in the number of hops in Figure 36a around 600-700 nodes for the STK data set. Figure 36b also has a sudden increment but it occurs around 200-300 nodes and it is less pronounced. Figure 36c has two sudden changes; Figure 36d has the same shape but its slope tends to be more constant. It seems that 
the built-up approach for this data set provokes this effect; after a certain number of nodes more nodes are reachable with almost the same number of hops. Note that Hybrid Timetable-Evolving Graph tends to output journeys with fewer hops while Pure-Timetable outputs journeys with more hops.

\section{STK: Running time}

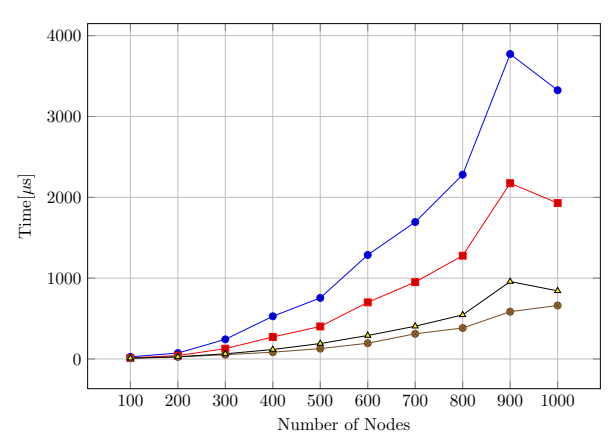

(a) $1000[\mathrm{~km}]$

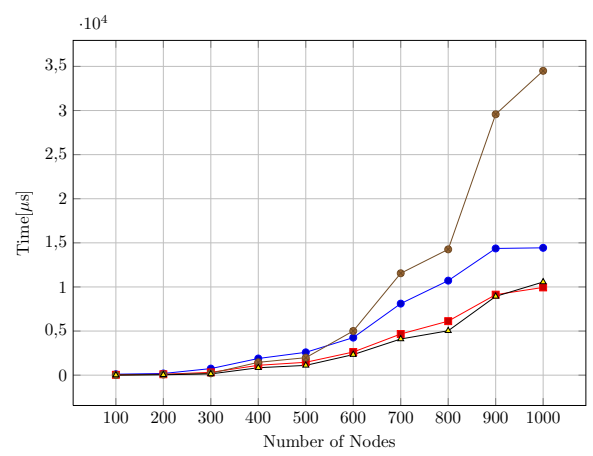

(c) $4000[\mathrm{~km}]$
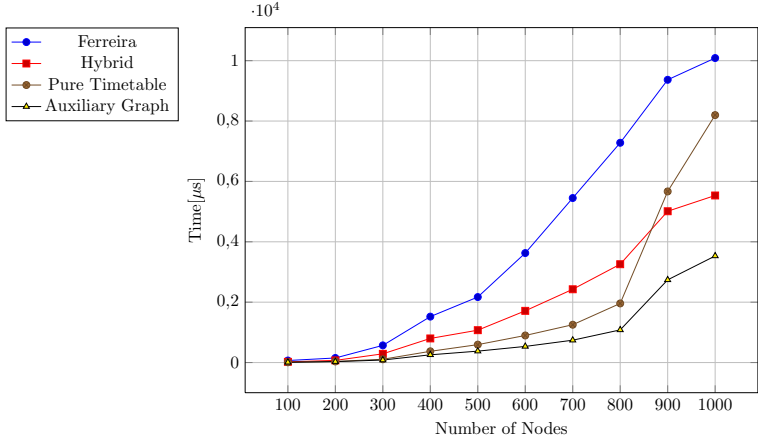

(b) $2500[\mathrm{~km}]$

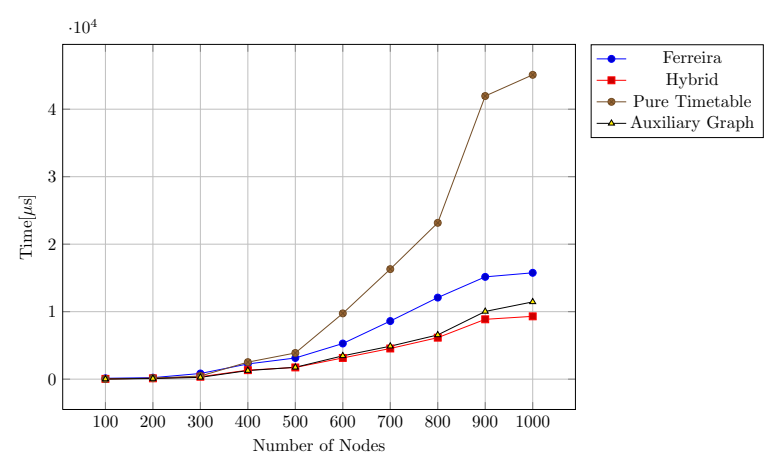

(d) $5500[\mathrm{~km}]$
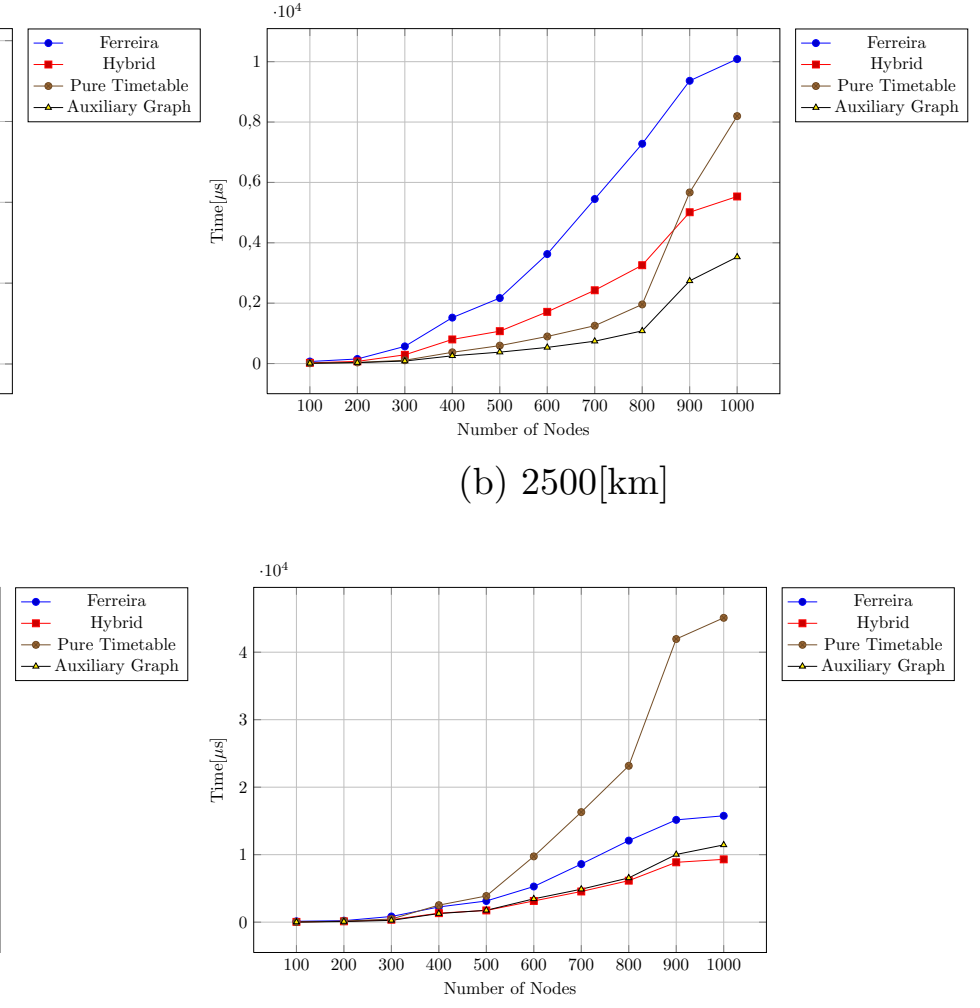

Figure 37: STK running time varying the number of nodes.

Figures $37 \mathrm{a}$ to $37 \mathrm{~d}$ show the running time of all 4 algorithms for networks with transmission range $1000 \mathrm{~km}, 2500 \mathrm{~km}, 4000 \mathrm{~km}$ and $5500 \mathrm{~km}$. We see that in all cases, and nearly all densities, Timetable with Auxiliary Graph has the best or close to the best running time. While for smaller transmission range, Pure-Timetable has the best running time no matter the node density, for larger transmission range, PureTimetable becomes the worst algorithm, and its running time increases sharply with the number of nodes. 


\subsubsection{Effect of varying the transmission range}

In this section, we keep the number of nodes fixed, and study the effect of varying the transmission range for each of our three data sets.

\section{SUMO: Number of hops}

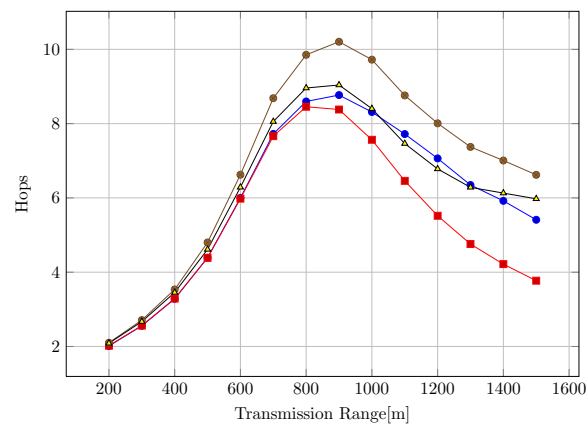

(a) 100 Nodes

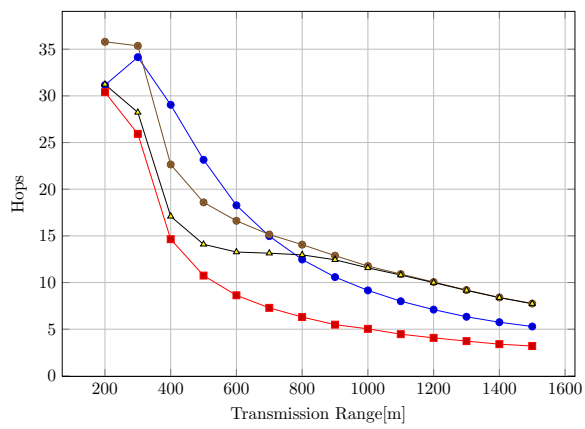

(c) 700 Nodes
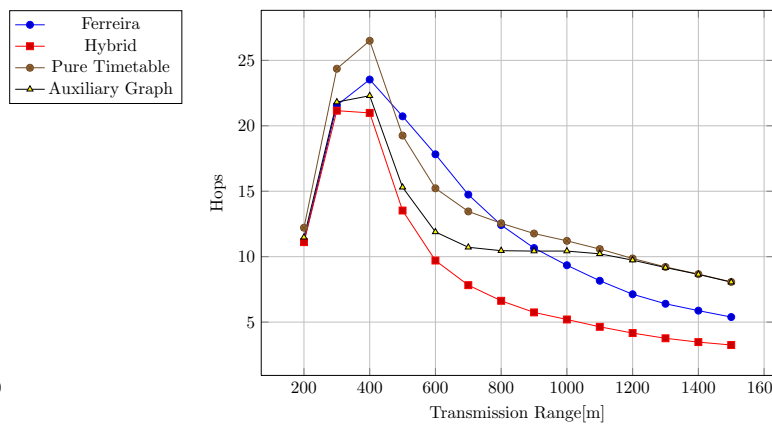

(b) 400 Nodes

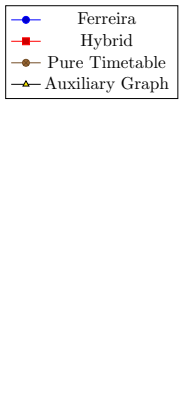

Figure 38: SUMO number of hops varying the transmission range.

Figures 38a to 40d show the effect of increasing the transmission range on the number of hops in journeys produced by the 4 algorithms. We see once again that Hybrid Timetable-Evolving Graph produces journeys with the fewest hops while PureTimetable generally produces journeys with more hops. An interesting observation is that for higher number of nodes, the number of hops decreases with transmission range for all algorithms, as one might expect. For 100 nodes and 400 nodes however, the number of hops in the journey first increases with increasing transmission range, and then decreases. This is likely because, for small number of nodes and small transmission range, many pairs of nodes are unreachable, As the transmission range grows, more pairs of nodes become reachable, with multi-hop paths. But as the range 
grows even more, all nodes become reachable, but the number of hops in the journeys starts decreasing.

\section{SUMO: Running time}

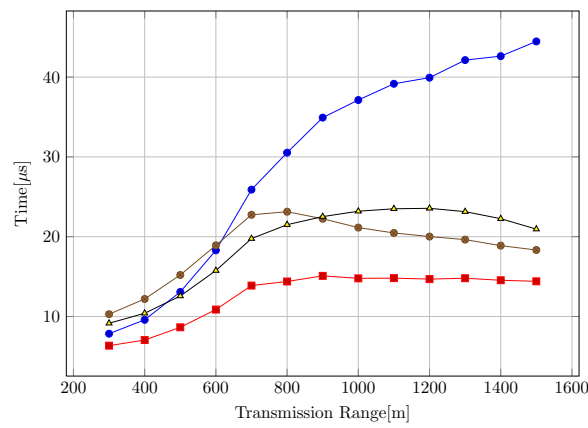

(a) 100 Nodes

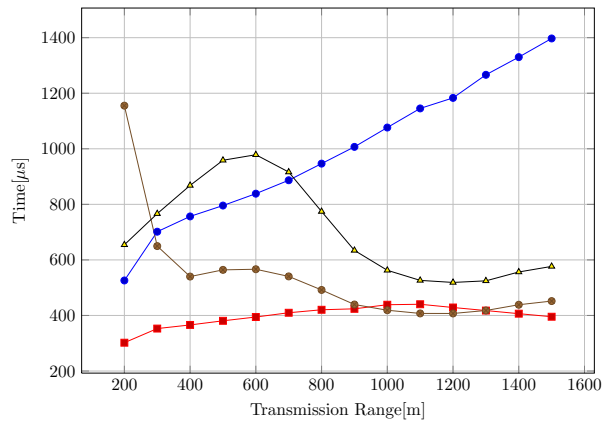

(c) 700 Nodes
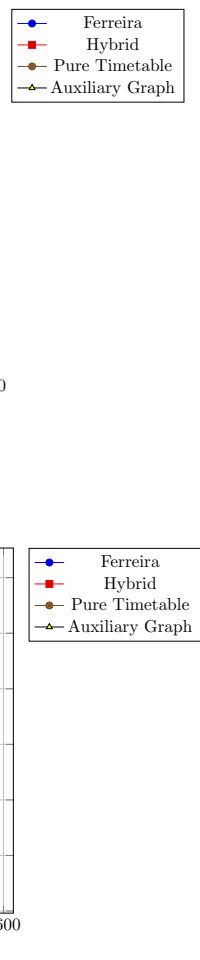

Figure 39: SUMO running time varying the transmission range.

Figures 39a to 39d show the running time of all 4 algorithms for networks of 100, 400, 700, and 1000 nodes respectively. We see that in all cases, as transmission range grows, all our algorithms outperform Ferreira's algorithm. In general, Hybrid Timetable-Evolving Graph has the best time for all transmission ranges and for all numbers of nodes studied, and the running time of all timetable-based algorithms appears to stabilise with transmission range as it increases, while Ferreira's algorithms takes more running time with increasing transmission range.

\section{STK: Number of hops}

Figure 40 shows the number of hops varying the transmission range in STK data set. As in the case of the SUMO data set, the number of hops first increases as we 


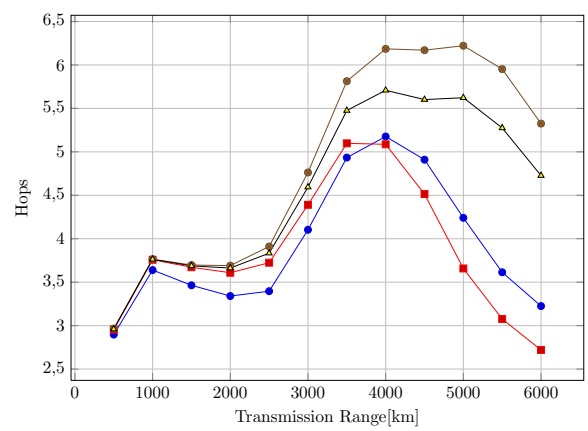

(a) 100 Nodes

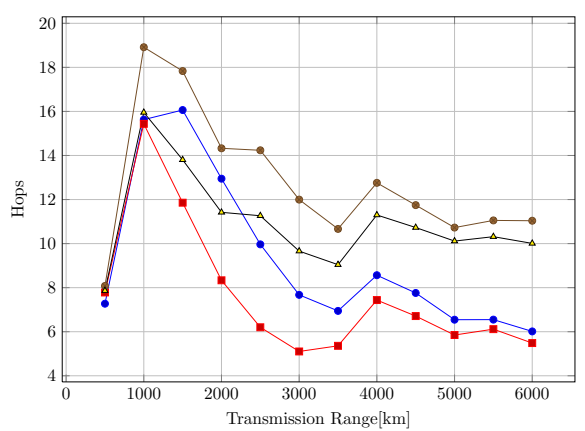

(c) 700 Nodes
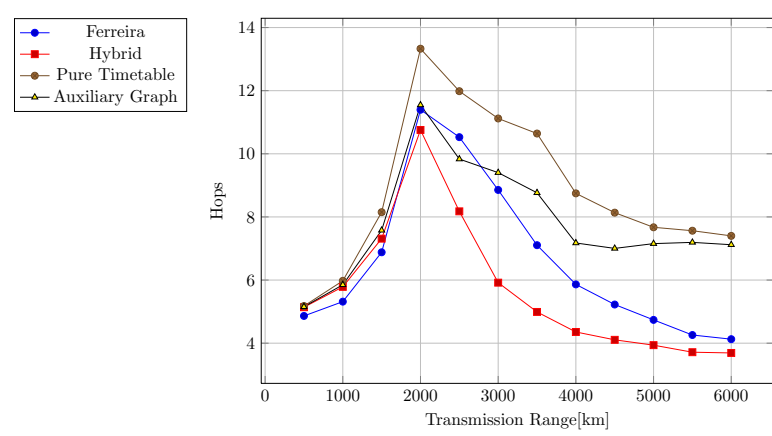

(b) 400 Nodes
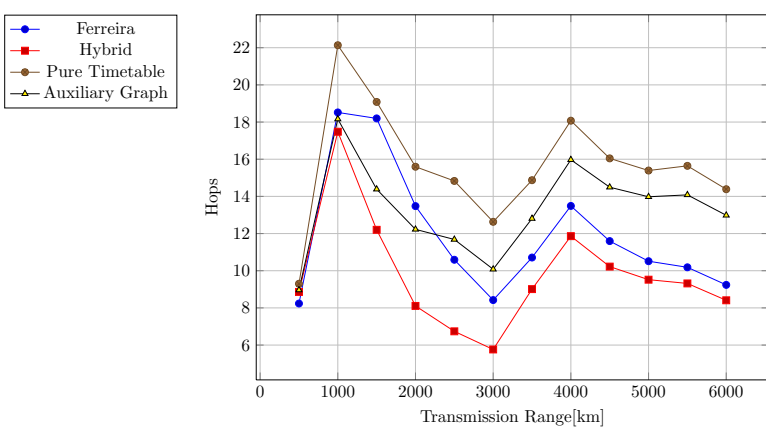

(d) 1000 Nodes
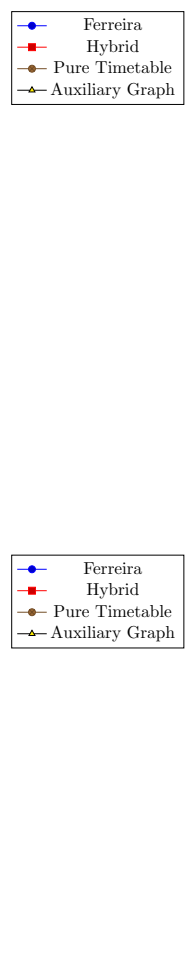

Figure 40: STK number of hops varying the transmission range.

increment the transmission range, and then later generally decreases. The peak is shifted left for higher number of nodes. A similar explanation holds as in the case of the SUMO data set; the curves are less smooth because this is not a random data set. Once again Hybrid Timetable-Evolving Graph produces journeys with the lowest number of hops.

\section{STK: Running time}

Figures 41a to 41d show the running time of all 4 algorithms for networks of 100, 400, 700, and 1000 nodes. We see that in all cases, and for nearly all transmission ranges, Timetable with Auxiliary Graph has the best running time. While PureTimetable works well for 100-node networks, as the number of nodes increases, the running time of Pure-Timetable degrades, especially for higher transmission ranges, becoming worse than Ferriera's approach. Hybrid Timetable-Evolving Graph also consistently has very good running time, becoming close to the time taken by Timetable with Auxiliary Graph for larger numbers of nodes. 


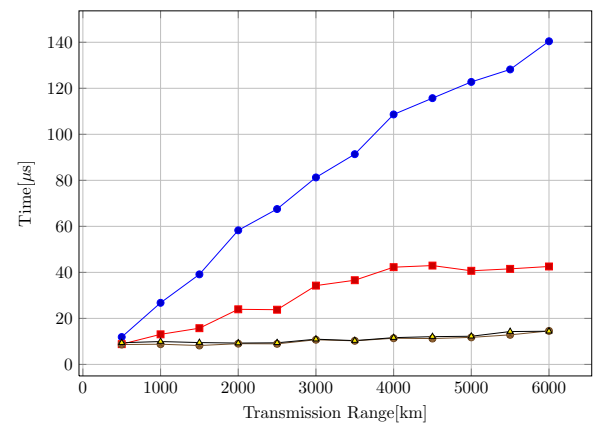

(a) 100 Nodes

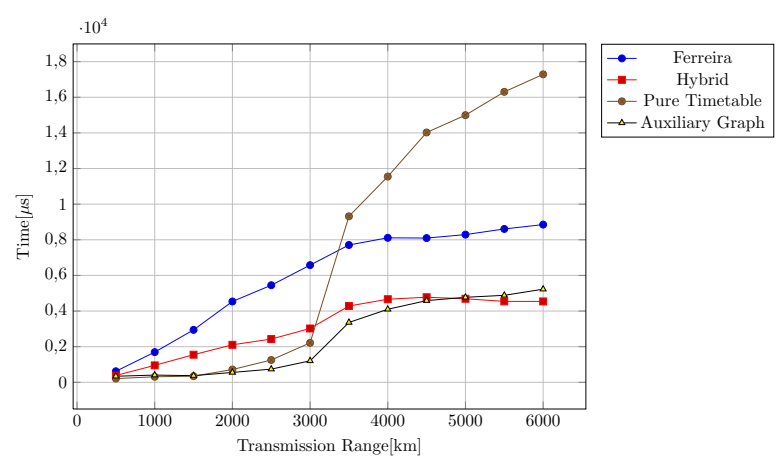

(c) 700 Nodes

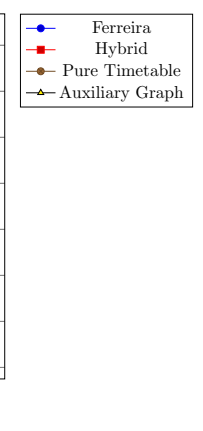

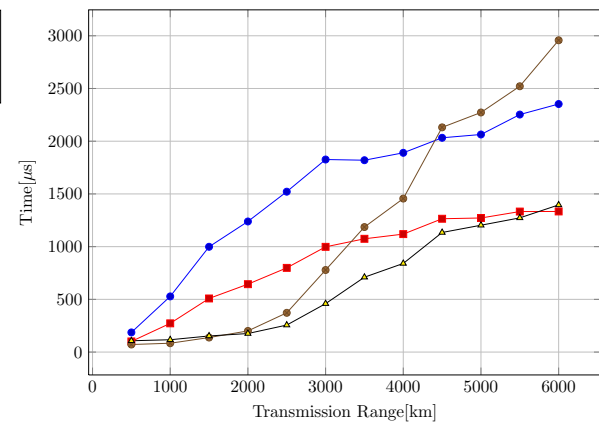

(b) 400 Nodes

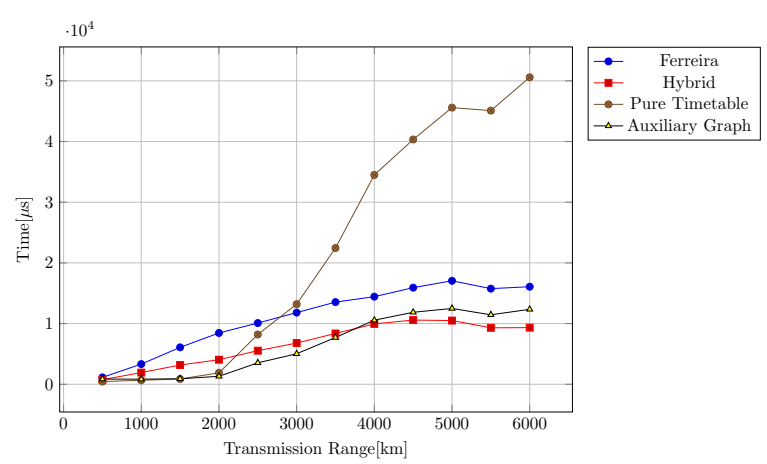

(d) 1000 Nodes

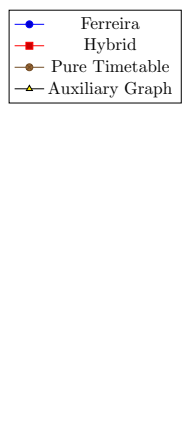




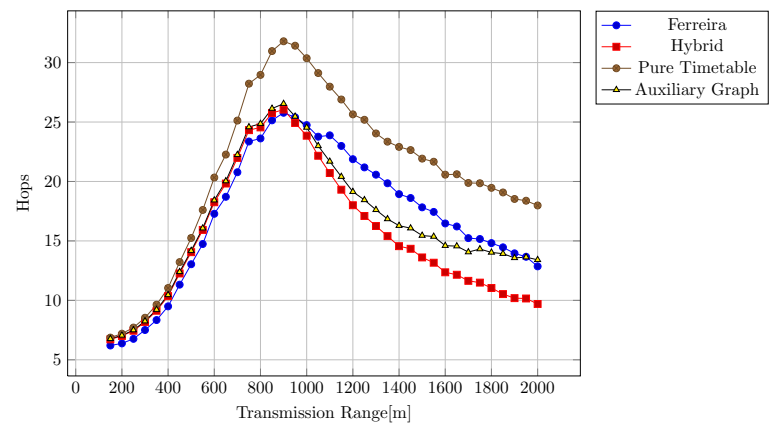

Figure 42: STM number of hops.

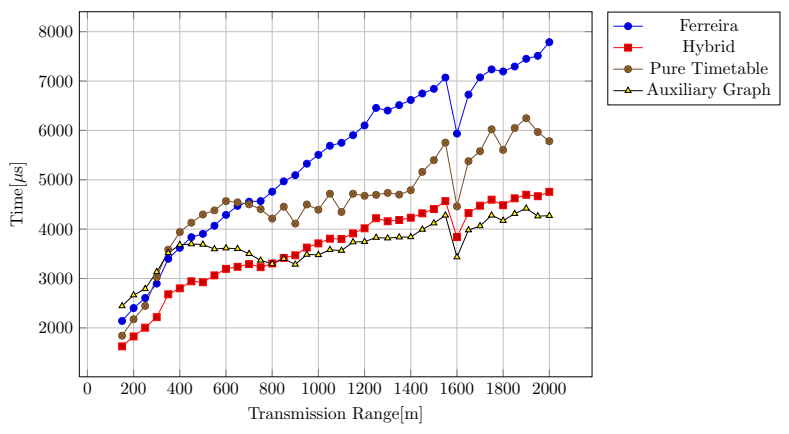

Figure 43: STM running time varying the transmission range.

than Ferreira's algorithm. For lower transmission ranges, Hybrid Timetable-Evolving Graph has the best running time, while for higher transmission ranges, Timetable with Auxiliary Graph has the best running time. 


\section{Chapter 6}

\section{Conclusions}

In this thesis, we studied the problem of finding the foremost journey between a source and target node in a FSDN. We proposed a new timetable-based approach for routing in FSDN, and described three variants of this approach. We proved the correctness of our algorithms and gave tight bounds on their complexity.

Our first algorithm, called Pure-Timetable, uses only a timetable data structure, and takes time $\mathcal{O}(|V||E|+|\mathcal{T}|)$. Our second algorithm, called Hybrid TimetableEvolving Graph, uses both a timetable data structure and the evolving graph data structure of [21], and takes time $\mathcal{O}(|E| \log I+|\mathcal{T}|+|V|)$. Our last algorithm, called Timetable with Auxiliary Graph, uses both a timetable data structure and a static graph data structure containing all edges currently active in the dynamic graph. It takes time $\mathcal{O}(|E|+|\mathcal{T}|+|V|)$. Here $|\mathcal{T}|$ is the total number of connections, and $I$ is the maximum number of connections corresponding to an edge.

We also validated the performance of our algorithms using extensive simulations run on SUMO, the STK satellite data set, and the STM bus network data set. Our results showed that our algorithms, particularly, Hybrid Timetable-Evolving Graph and Timetable with Auxiliary Graph are faster than the previous algorithm given by Ferreira [21] for almost all sizes of network and all transmission ranges. We also showed that Hybrid Timetable-Evolving Graph produces journeys with fewer hops than the other algorithms.

We considered no delay for edge traversal since the minimum duration of a connection is big enough to consider propagation delay negligible in our setting. In future work, it would be interesting to modify our algorithms for the case when a connection 
takes a certain number of time steps to be traversed. Also, there was no propagation model [2] for the wireless communication between vehicles, buses and satellites. It would be interesting to experiment with the speed and type of the vehicles and pick one propagation model in SUMO simulations to see if they impact the journey characteristics. 


\section{Bibliography}

[1] P. Alvarez, M. Behrisch, L. Bieker-Walz, J. Erdmann, Y. Flötteröd, R. Hilbrich, L. Lücken, J. Rummel, P. Wagner, and E. Wießner. Microscopic traffic simulation using SUMO. In The 21st IEEE International Conference on Intelligent Transportation Systems. IEEE, 2018.

[2] V. S. Anusha, G. Nithya, and S. Rao. A comprehensive survey of electromagnetic propagation models. In 2017 International Conference on Communication and Signal Processing (ICCSP), pages 1457-1462. IEEE, 2017.

[3] M. Y. Arafat and S. Moh. Routing protocols for unmanned aerial vehicle networks: A survey. IEEE Access, 7:99694-99720, 2019.

[4] S. Bakhtiari. ACATS project final report. Technical report, Canadian Urban Transit Research and Innovation Consortium, 2020.

[5] M. Besta, M. Fischer, V. Kalavri, M. Kapralov, and T. Hoefler. Practice of streaming and dynamic graphs: Concepts, models, systems, and parallelism. Computing Research Repository (CoRR), 2020.

[6] M. Bläser. Fast matrix multiplication. Theory of Computing, pages 1-60, 2013.

[7] F. Busato, O. Green, N. Bombieri, and D. Bader. Hornet: An efficient data structure for dynamic sparse graphs and matrices on GPUs. In 2018 IEEE High Performance extreme Computing Conference (HPEC), pages 1-7. IEEE, 2018.

[8] J. Byun, S. Woo, and D. Kim. Chronograph: Enabling temporal graph traversals for efficient information diffusion analysis over time. IEEE Transactions on Knowledge and Data Engineering, 32(3):424-437, 2020. 
[9] A. Casteigts, P. Flocchini, W. Quattrociocchi, and N. Santoro. Time-varying graphs and dynamic networks. Intl. Journal of Parallel, Emergent and Distributed Systems, 27(5):387-408, 2012.

[10] S. Chaudhary, N. Chaudhary, S. Sharma, and B Choudhary. High speed intersatellite communication system by incorporating hybrid polarization-wavelength division multiplexing scheme. Journal of Optical Communications, 39(1):87-92, 2017.

[11] Ansys Company. Systems tool kit (STK). https://www.agi.com, 2020. Windows version 12.0 .

[12] Google Technology Company. GTFS realtime overview. https://developers. google.com/transit/gtfs-realtime, Jul 2019. [Online] August 12, 2020.

[13] F. Cunha, L. Villas, A. Boukerche, G. Maia, A. Viana, R. Mini, and A. Loureiro. Data communication in VANETs: Protocols, applications and challenges. Ad Hoc Networks, 44:90-103, 2016.

[14] Société de transport de Montréal. Developers. http://www.stm.info/en/ about/developers. [Online] accessed August 12, 2020.

[15] J. Dibbelt, T. Pajor, B. Strasser, and D. Wagner. Connection scan algorithm. Journal of Experimental Algorithmics (JEA), 23:1-56, 2018.

[16] E. Dijkstra et al. A note on two problems in connexion with graphs. Numerische mathematik, 1(1):269-271, 1959.

[17] EasyMile. Ez10 passenger shuttle. https://easymile.com/vehicle-solutions/ez10passenger-shuttle. [Online] accessed March 1, 2021.

[18] D. Ediger, R. McColl, J. Riedy, and D. Bader. Stinger: High performance data structure for streaming graphs. In 2012 IEEE Conference on High Performance Extreme Computing, pages 1-5. IEEE, 2012.

[19] E. Ekici, I. F. Akyildiz, and M. D. Bender. Datagram routing algorithm for LEO satellite networks. In Proceedings IEEE INFOCOM 2000. Conference on Computer Communications. Nineteenth Annual Joint Conference of the IEEE 
Computer and Communications Societies (Cat. No.00CH37064), volume 2, pages 500-508 vol.2, 2000.

[20] W. Fang, M. Mukherjee, L. Shu, Z. Zhou, and G. P. Hancke. Energy utilization concerned sleep scheduling in wireless powered communication networks. In 2017 IEEE International Conference on Communications Workshops (ICC Workshops), pages 558-563, 2017.

[21] A. Ferreira. On models and algorithms for dynamic communication networks: The case for evolving graphs. In Proc. ALGOTEL, 2002.

[22] O. Green. High performance computing for irregular algorithms and applications with an emphasis on big data analytics. $\mathrm{PhD}$ thesis, Georgia Institute of Technology, 2014.

[23] O. Green and D. Bader. custinger: Supporting dynamic graph algorithms for GPUs. In 2016 IEEE High Performance Extreme Computing Conference (HPEC), pages 1-6. IEEE, 2016.

[24] O. Green, R. McColl, and D. Bader. A fast algorithm for streaming betweenness centrality. In 2012 International Conference on Privacy, Security, Risk and Trust and 2012 International Confernece on Social Computing, pages 11-20. IEEE, 2012.

[25] W. Huo. Query processing on temporally evolving social data. PhD thesis, UC Riverside, 2013.

[26] W. Huo and V. Tsotras. Efficient temporal shortest path queries on evolving social graphs. In Proceedings of the 26th International Conference on Scientific and Statistical Database Management, pages 1-4, 2014.

[27] Transdev Canada Inc. Autonomous mobility. https://www.transdev.ca/en/oursolutions/autonomous-mobility/, Jul 2020. [Online] accessed March 1, 2021.

[28] G. Khanna, S. K. Chaturvedi, and S. Soh. On computing the reliability of opportunistic multihop networks with mobile relays. Quality and Reliability Engineering International, 35(4):870-888, 2019. 
[29] G. Khanna, S. Soh, S. K. Chaturvedi, and K. Chin. On enumeration of spanning arborescences and reliability for network broadcast in fixed-schedule dynamic networks. IEEE Transactions on Network Science and Engineering, 2020.

[30] U. Khurana and A. Deshpande. Efficient snapshot retrieval over historical graph data. In 2013 IEEE 29th International Conference on Data Engineering (ICDE), pages 997-1008. IEEE, 2013.

[31] K. Kiela, V. Barzdenas, M. Jurgo, V. Macaitis, J. Rafanavicius, A. Vasjanov, L. Kladovscikov, and R. Navickas. Review of V2X-IoT standards and frameworks for its applications. Applied Sciences, 10(12):4314, 2020.

[32] G. Koloniari and K. Stefanidis. Social search queries in time. In Proc. 7th Workshop Personalized Access, Profile Manage., Context Awareness Databases, pages 1-4, 2013.

[33] A. Kosmatopoulos, A. Gounaris, and K. Tsichlas. Hinode: implementing a vertex-centric modelling approach to maintaining historical graph data. Computing, 101(12):1885-1908, 2019.

[34] A. G. Labouseur, J. Birnbaum, P. W. Olsen, S. R. Spillane, J. Vijayan, J. Hwang, and W. Han. The $\mathrm{G}^{*}$ graph database: efficiently managing large distributed dynamic graphs. Distributed and Parallel Databases, 33(4):479-514, 2015.

[35] I. Maduako and M. Wachowicz. A space-time varying graph for modelling places and events in a network. International Journal of Geographical Information Science, 33(10):1915-1935, 2019.

[36] Y. Miao, W. Han, K. Li, M. Wu, F. Yang, L. Zhou, V. Prabhakaran, E. Chen, and W. Chen. Immortalgraph: A system for storage and analysis of temporal graphs. ACM Transactions on Storage (TOS), 11(3):1-34, 2015.

[37] Z. H. Mir and F. Filali. LTE and IEEE 802.11p for vehicular networking: a performance evaluation. EURASIP Journal on Wireless Communications and Networking, 2014(1):1-15, 2014.

[38] V. Z. Moffitt and J. Stoyanovich. Towards sequenced semantics for evolving graphs. In EDBT, pages 446-449, 2017. 
[39] M. Nasimi, M. A. Habibi, and H. D. Schotten. Platoon-assisted vehicular cloud in VANET: Vision and challenges. arXiv preprint arXiv:2008.10928, 2020.

[40] R. A. Nazib and S. Moh. Routing protocols for unmanned aerial vehicle-aided vehicular Ad Hoc networks: A survey. IEEE Access, 8:77535-77560, 2020.

[41] P. Ni, M. Hanai, W. J. Tan, and W. Cai. Efficient closeness centrality computation in time-evolving graphs. In Proceedings of the 2019 IEEE/ACM International Conference on Advances in Social Networks Analysis and Mining, pages 378-385, 2019.

[42] A. Pentland, R. Fletcher, and A. Hasson. DakNet: rethinking connectivity in developing nations. Computer, 37(1):78-83, 2004.

[43] C. Ren, E. Lo, B. Kao, X. Zhu, and R. Cheng. On querying historical evolving graph sequences. Proceedings of the VLDB Endowment, 4(11):726-737, 2011.

[44] C. Ren, E. Lo, B. Kao, X. Zhu, R. Cheng, and D. W. Cheung. Efficient processing of shortest path queries in evolving graph sequences. Information Systems, 70:18$31,2017$.

[45] R. A. Rossi, B. Gallagher, J. Neville, and K. Henderson. Modeling dynamic behavior in large evolving graphs. In Proceedings of the sixth ACM international conference on Web search and data mining, pages 667-676, 2013.

[46] B. Schäling. The boost $C++$ libraries, chapter 38: Boost.Timer. Boris Schäling, 2011.

[47] D. Sengupta and S. L. Song. Evograph: On-the-fly efficient mining of evolving graphs on GPU. In International Supercomputing Conference, pages 97-119. Springer, 2017.

[48] V. R. Syrotiuk and C. J. Colbourn. Routing in mobile aerial networks. In WiOpt'03: Modeling and Optimization in Mobile, Ad Hoc and Wireless Networks, pages 9-pages, 2003.

[49] A. Tripathy and O. Green. Scaling betweenness centrality in dynamic graphs. In 2018 IEEE High Performance extreme Computing Conference (HPEC), pages 1-7. IEEE, 2018. 
[50] K. Wehmuth, A. Ziviani, and E. Fleury. A unifying model for representing timevarying graphs. In 2015 IEEE International Conference on Data Science and Advanced Analytics (DSAA), pages 1-10. IEEE, 2015.

[51] M. Winter, R. Zayer, and M. Steinberger. Autonomous, independent management of dynamic graphs on GPUs. In 2017 IEEE High Performance Extreme Computing Conference (HPEC), pages 1-7. IEEE, 2017.

[52] L. Xiangyu, L. Yingxiao, G. Xiaolin, and Y. Zhenhua. An efficient snapshot strategy for dynamic graph storage systems to support historical queries. IEEE Access, 8:90838-90846, 2020.

[53] B. Xuan, A. Ferreira, and A. Jarry. Computing shortest, fastest, and foremost journeys in dynamic networks. International Journal of Foundations of Computer Science, 14(02):267-285, 2003.

[54] Y. Yang, J. X. Yu, H. Gao, J. Pei, and J. Li. Mining most frequently changing component in evolving graphs. World Wide Web, 17(3):351-376, 2014.

[55] A. Zaki, M. Attia, D. Hegazy, and S. Amin. Efficient distributed dynamic graph system. In 2015 IEEE Seventh International Conference on Intelligent Computing and Information Systems (ICICIS), pages 465-471. IEEE, 2015.

[56] A. Zaki, M. Attia, D. Hegazy, and S. Amin. Comprehensive survey on dynamic graph models. International Journal of Advanced Computer Science and Applications, 7(2):573-582, 2016.

[57] M. Zameni, M. Moshtaghi, and C. Leckie. Efficient query processing on road traffic network. In 2016 IEEE International Conference on Pervasive Computing and Communication Workshops (PerCom Workshops), pages 1-6. IEEE, 2016.

[58] Z. Zhang, C. Jiang, S. Guo, Y. Qian, and Y. Ren. Temporal centrality-balanced traffic management for space satellite networks. IEEE Transactions on Vehicular Technology, 67(5):4427-4439, 2017. 